\title{
REDUCED COMPLEXITY FREQUENCY DOMAIN TURBO EQUALIZATION
}

\author{
by \\ Shufen Sun

\begin{abstract}
A thesis submitted to
the Faculty of Graduate Studies and Research

in partial fulfillment of the requirements for the degree of
\end{abstract}

\section{Master of Applied Science}
Ottawa-Carleton Institute for Electrical and Computer Engineering
Department of System and Computer Engineering

\author{
Carleton University \\ Ottawa, Ontario, Canada \\ January 12, 2004 \\ CC Copyright 2004, Shufen Sun
}




\author{
National Library \\ of Canada \\ Acquisitions and \\ Bibliographic Services \\ 395 Wellington Street \\ Ottawa ON K1A ON4 \\ Canada
}

Bibliothèque nationale

du Canada

Acquisisitons et services bibliographiques

395 , rue Wellington Ottawa ON K1A ON4 Canada
Your file Votre référence ISBN: 0-612-93949-9

Ourfile Notre référence ISBN: 0-612-93949-9
The author has granted a nonexclusive licence allowing the National Library of Canada to reproduce, loan, distribute or sell copies of this thesis in microform, paper or electronic formats.

The author retains ownership of the copyright in this thesis. Neither the thesis nor substantial extracts from it may be printed or otherwise reproduced without the author's permission.
L'auteur a accordé une licence non exclusive permettant à la Bibliothèque nationale du Canada de reproduire, prêter, distribuer ou vendre des copies de cette thèse sous la forme de microfiche/film, de reproduction sur papier ou sur format électronique.

L'auteur conserve la propriété du droit d'auteur qui protège cette thèse. $\mathrm{Ni}$ la thèse ni des extraits substantiels de celle-ci ne doivent être imprimés ou aturement reproduits sans son autorisation.
In compliance with the Canadian Privacy Act some supporting forms may have been removed from this dissertation.

While these forms may be included in the document page count, their removal does not represent any loss of content from the dissertation.
Conformément à la loi canadienne sur la protection de la vie privée, quelques formulaires secondaires ont été enlevés de ce manuscrit.

Bien que ces formulaires aient inclus dans la pagination, il n'y aura aucun contenu manquant. 


\begin{abstract}
This thesis investigates the performance of turbo equalizers based on interference canceller (IC) that has been implemented in the frequency domain (FD-IC), as opposed to the more typical time domain implementation (TD-IC). A computational complexity analysis shows that the FD-IC has much lower complexity when equalizing channels with long delay spreads, while still maintaining the excellent performance of the TDIC. A novel, sub-optimal, reduced-complexity hybrid frequency/time domain interference canceller (hybrid IC), which is based on implementing part of the IC in the frequency domain (the $P$-filter), and part in the time domain (the $q$-filter), is proposed. The filter coefficients of the hybrid IC are derived according to the minimum mean squared error criterion. It is shown by means of computer simulation that turbo equalization using the hybrid IC can achieve respectable performance in terms of the bit and frame error rates, without the need for performing FFT's at each iteration, thereby providing reduced computational complexity. Although the hybrid IC is suboptimal as compared to the FD-IC, in some cases it is shown that it can even provide slightly better performance than the FD-IC.
\end{abstract}




\section{ACKNOWLEDGEMENTS}

I would like to thank my two thesis supervisors, Professor Ian Marsland and Professor David Falconer, for their excellent guidance, valuable ideas, suggestions, and constructive discussions, patience and encouragement to make this thesis possible.

I also would like to express my thanks to Ms. Xiaobin Tang, Ms. Shermeen Nizami, Mr. Xiaojing Tao and Mr. Abiola Adegboyega in my lab for their help while doing my thesis.

In addition, I thank Ms. Blazenka Power, the graduate assistant of my department, for her help and supports.

I am also very grateful for my husband Fang and my daughter Pelagia for their encouragement and supports. Last but not least, I really appreciate my parents and my sisters and brother for their emotional supports during my study. 


\section{TABLE OF CONTENTS}

$\begin{array}{ll}\text { Abstract } & \text { ii }\end{array}$

Acknowledgement iii

Table of Contents iv

List of Figure vii

List of Tables $\quad$ x

List of Acronyms and Abbreviations xi

$\begin{array}{lll}\text { Chapter } 1 \text { INTRODUCTION } & 1\end{array}$

1.1 Background 1

$\begin{array}{ll}1.2 & \text { Thesis Motivation }\end{array}$

1.3 Thesis Objectives 3

1.4 Thesis Contributions 4

1.5 Thesis Organization 4

$\begin{array}{ll}\text { Chapter } 2 & \text { SYSTEM MODEL AND REVIEW OF FREQUENCY } \\ & \text { DOMAIN LINEAR EQUALIZATION }\end{array}$

$\begin{array}{lll}2.1 & \text { Introduction } & 6\end{array}$

$\begin{array}{lll}2.2 & \text { Transmitter } & 7\end{array}$

2.2.1 Convolutional Encoder $\quad 8$

2.2.2 Interleaver $\quad 8$

2.2.3 Symbol Mapper $\quad 9$

2.3 Discrete-time ISI Channel Model 9

$\begin{array}{ll}\text { 2.3.1 Pulse Shaping Filter } & 10\end{array}$

$\begin{array}{ll}\text { 2.3.2 Modulator } & 10\end{array}$ 
2.3.5 Receive Filter

2.3.6 Symbol-rate Symbol Sampler

$2.4 \quad$ Receiver

2.4.1 Equalizer

2.4.2 Deinterleaver

2.4.3 MAP Decoder

2.5 Frequency Domain Linear Equalizer

Chapter 3 FREQUENCY DOMAIN TURBO EQUALIZATION

3.1 Introduction to Turbo Equalization

3.1.1 Interference Canceller

3.1.2 IC-based Turbo Equalization

3.2 Turbo Equalization Using Frequency Domain IC

3.3 Reduced Complexity Frequency Domain IC

3.3.1 Motivation

3.3.2 Coefficients of the hybrid IC 26

3.3.3 Mean and variance of the IC output

3.4 Complexity Comparison

3.4.1 Complexity of LE

3.4.2 Complexity of the IC

3.4.3 Complexity of the hybrid IC 
4.1 Performance of FD-IC over Static ISI Channels 39

4.1.1 Proakis B Channel $\quad 40$

4.1.2 Proakis C Channel $\quad 40$

4.1.3 Two-tap Channel with a Long Delay Spread 43

4.2 Performance over Rayleigh Frequency-Selective Fading Channels 48

4.2.1 SUI-5 and SUI-6 Channels 48

4.2.2 10-tap Fading Channel $\quad 54$

4.3 Performance over the hybrid IC 58

4.3.1 Performance over Static Channels $\quad 58$

4.3.2 Performance over the SUI-5 Channel 61

4.3.3 Performance over 10-tap Fading Channel 61

Chapter 5 CONCLUSIONS AND FUTURE RESEARCH 66

5.1 Summary and Discussion $\quad 66$

$\begin{array}{lll}5.2 & \text { Further Research } & 68\end{array}$

$\begin{array}{ll}\text { LIST OF REFERENCES } & \mathbf{7 0}\end{array}$

Appendix A Coefficients, Mean and Variance For the Original IC

Turbo Equalization MAP Algorithm $\quad 73$

Appendix B Coefficients, Mean and Variance for Hybrid Reduced

Complexity Turbo Equalization Coefficients of LE 


\section{List of Figures}

Figure 2-1 Block diagram of the communication system 6

Figure 2-2 Structure of the transmitter $\quad 7$

Figure 2-3 Discrete-time ISI channel model 9

$\begin{array}{lll}\text { Figure 2-4 Structure of receiver } & 14\end{array}$

$\begin{array}{lll}\text { Figure 2-5 Frequency domain linear equalizer } & 17\end{array}$

$\begin{array}{lll}\text { Figure 3-1 Block diagram of an interference canceller } & 20\end{array}$

Figure 3-2 Block diagram of IC-based turbo equalizer 21

Figure 3-3 Structure of the frequency domain IC 25

Figure 3-4 Structure of the hybrid IC 26

Figure 3-5 Computational complexity of the TD-IC, FD-IC and hybrid IC 38

Figure 4-1 Impulse and frequency response of the Proakis B channel 41

Figure 4-2 BER performance of the FD-IC over the Proakis B channel 42

Figure 4-3 FER performance of the FD-IC over the Proakis B channel 42

Figure 4-4 Impulse and frequency response of the Proakis C channel 44

Figure 4-5 BER performance of the FD-IC over the Proakis C channel 45

Figure 4-6 FER performance of the FD-IC over the Proakis C channel 45 
Figure 4-7 Impulse and frequency response of a 2-tap channel with long delay spread

Figure 4-8 BER performance of the FD-IC over 2-tap channel with long delay spread

Figure 4-9 FER performance of the FD-IC over 2-tap channel with long delay spread

Figure 4-10 Delay spread profile for the SUI-5 channel

Figure 4-11 Delay spread profile for the SUI-6 channel

Figure 4-12 BER performance of the FD-IC for QPSK over the SUI-5 channel 51

Figure 4-13 FER performance of the FD-IC for QPSK over the SUI-5 channel 51

Figure 4-14 BER performance of the FD-IC for QPSK over the SUI-6 channel 52

Figure 4-15 FER performance of the FD-IC for QPSK over the SUI-6 channel 52

Figure 4-16 BER performance of the FD-IC for 16-QAM over the SUI-5 channel

Figure 4-17 FER performance of the FD-IC for 16-QAM over the SUI-5 channel

Figure 4-18 BER performance of the FD-IC for QPSK over 10-tap fading channel

Figure 4-19 FER performance of the FD-IC for QPSK over 10-tap fading channel 
Figure 4-20 BER performance of the FD-IC for QPSK over 10-tap fading channel (with 10th iteration)

Figure 4-21 FER performance of the FD-IC for QPSK over 10-tap fading Channel (with 10th iteration)

Figure 4-22 BER performance of the FD-IC for 16-QAM over 10-tap fading channel

Figure 4-23 FER performance of the FD-IC for 16-QAM over 10-tap fading channel

Figure 4-24 BER of the hybrid IC for QPSK over the Proakis B channel

Figure 4-25 FER of the hybrid IC for QPSK over the Proakis B channel

Figure 4-26 BER of the hybrid IC for QPSK over the Proakis C channel

Figure 4-27 FER of the hybrid IC for QPSK over the Proakis C channel

Figure 4-28 BER of the hybrid for QPSK over the SUI-5 channel

Figure 4-29 FER of the hybrid for QPSK over the SUI-5 channel

Figure 4-30 BER of the hybrid for QPSK over 10-tap fading channel

Figure 4-31 FER of the hybrid for QPSK over 10-tap fading channel

Figure 4-32 BER of the hybrid for 16-QAM over 10-tap fading channel

Figure 4-33 FER of the hybrid for 16-QAM over 10-tap fading channel 


\section{List of Tables}

Table 3-1 Complexity per symbol of one FFT 31

Table 3-2 Complexity per symbol of TD-LE 31

Table 3-3 Complexity per symbol of FD-LE 31

Table 3-4 Complexity of the $p$-filter in the TD-IC 33

Table 3-5 Complexity of the $q$-filter in the TD-IC 33

Table 3-6 Complexity of the $P$-filter in the FD-IC 33

Table 3-7 Complexity of the $Q$-filter in the FD-IC 34

Table 3-8 Complexity of the TD-IC 34

Table 3-9 Complexity of the FD-IC 34

Table 3-10 Complexity of the $P$-filter in the hybrid IC 36

Table 3-11 Complexity of the $q$-filter in the hybrid IC 36

Table 3-12 Complexity of the hybrid IC 36

Table 3-13 Complexity comparison $\quad 37$

Table 4-1 Parameters for the SUI-5 and SUI-6 channels 49 


\section{List of Acronyms and Abbreviations}

APP

AWGN

BER

$\mathrm{dB}$

DFE

DFT

FER

FFT

FD-IC

$\mathrm{Hz}$

IC

IFFT

ISI

LE

MAP

$\mathrm{MCU}$

MF

MLSE

MMSE

MMSE-LE
A Posteriori Probability

Additive White Gaussian Noise

Bit Error Rate

Decibel

Decision Feedback Equalizer

Discrete Fourier Transform

Frame Error Rate

Fast Fourier Transform

Frequency Domain Interference Canceller

Hertz

Interference Canceller

Inverse Fast Fourier Transform

Intersymbol Interference

Linear Equalizer

Maximum A Posteriori Probability

Metric Calculation Unit

Matched Filter

Maximum Likelihood Sequence Estimation

Minimum Mean Square Error

Minimum Mean Square Error Linear Equalizer 
QAM

QPSK

SNR

TCM

TD-IC
Quadrature Amplitude Modulation

Quadrature Phase Shift Keying

Signal to Noise Ratio

Trellis Coded Modulation

Time Domain Interference Canceller 


\section{Chapter 1}

\section{INTRODUCTION}

\subsection{Background}

In wireless communication systems, the transmitted signals simultaneously travel over multipaths to the receiver, with the signal arriving along each path suffering from different attenuations and propagation delays. This multipaths interference causes time dispersion of the transmitted signals, results in intersymbol interference (ISI), or if the transmission environment is time-variant, frequency selective fading. To mitigate the detrimental effects of ISI, equalization is widely employed.

Traditionally, equalization techniques include the linear equalizer, decision feedback equalizer (DFE), maximum likelihood sequence estimation (MLSE), etc. During the past decade, significant attention has been placed on turbo equalization. Turbo equalization was motivated by the iterative decoding algorithm for turbo codes [1]-[6] and was first proposed by Douillard et al. in 1995 [7]. Traditionally, equalization and channel decoding are considered separately [8]-[10]. However, with turbo equalization, the equalization and channel decoding are combined through an iterative process and jointly optimized so that the whole receiver performance is enhanced. The performance of turbo equalization has been shown to be near optimum over many transmission channels. So far, turbo equalization has been proposed for many applications, such as in wireless cellular systems [11], diversity channels [12] and trellis coded modulation (TCM) $[13,14]$. For the equalizer, different equalization schemes are employed, such as maximum a posteriori probability (MAP) equalization 
[7, 15], linear equalizer (LE) [16] and interference canceller (IC) [17]. Since turbo equalization based on the IC has low computational complexity, much research has been placed on it [17]-[20].

\subsection{Thesis motivation}

IC-based turbo equalization was originally proposed in by Glavieux et al. [17] in 1997. The IC consists of two filters, the $p$-filter and the $q$-filter. The purpose of the $p$ filter is to enhance the received signal-to-noise ratio (SNR), and the purpose of the $q$ filter is to eliminate the ISI. The input to the $P$-filter is the received samples from the output of a filter matched to the transmitted pulse shape. Ideally, the input to the $q$ filter would be the actual transmitted symbols, but in practice these symbols are not known at the receiver. With IC-based turbo equalization, the received data is first processed with a linear equalizer, which partially suppresses the ISI. The equalizer output is passed to the decoder which decodes the convolutional code. Using the output of the decoder it is possible to locally generate estimates of the transmitted data. These estimates are used as the input to the $q$-filter of the IC to more effectively eliminate the ISI. The IC output is then passed back to the decoder, which again decodes the convolutional code, this time more reliably since there is less residual ISI in the input. This process is repeated in an iterative (turbo) fashion. With the originally proposed system the IC was implemented in the time domain, but more recently Tuchler and Hagenaeur proposed a frequency domain implementation [21]. With this system, the two filters are implemented in the frequency domain, using the fast Fourier transform (FFT) to convert to and from the frequency domain. For channels with long delay spreads, the frequency domain system has lower implementation complexity than the 
time-domain version. However, since the frequency-domain IC requires an FFT and an inverse FFT (IFFT) for each iteration of the turbo equalization loop, the complexity still remains quite high. It is desirable to further reduce the complexity by eliminating the need for FFT's within the loop. In this thesis we propose a novel hybrid frequency/time domain interference canceller (hybrid IC), which is based on implementing the $P$-filter in the frequency domain, and the $q$-filter in the time domain. By carefully calculating the optimal coefficients of these two filters, respectable performance is possible without the need for performing FFT's at each iteration.

\subsection{Thesis objectives}

The first objective of this thesis is to investigate whether the IC-based turbo equalization in frequency domain can improve the system performance over channels with long delay spreads, sometimes which was not investigated in the literature [21, $22]$.

The second objective is to find an approach to further reduce the computational complexity of IC-based turbo equalizers. If $q$-filter in the IC operating in time domain only uses the several largest pairs of $q$-filter coefficients instead of all coefficients when the length of channel response is long and the majority of its values are very small, what will happen to the whole performance? A hybrid reduced complexity IC, which makes use of both advantages of time domain and frequency domain and simplifies the receiver's implementation, is proposed in this thesis.

The third objective is to investigate the performances of this hybrid IC over both static ISI channels and frequency selective fading channels. 


\subsection{Thesis contributions}

In this thesis, there are several contributions as follows:

1. Simulations verify that IC-based turbo equalization in the frequency domain can improve bit error rate (BER) and frame error rate (FER) performance for channels with long delay spreads.

2. The filter coefficients in the proposed hybrid IC are derived according to the minimum mean squared error criterion.

3. The proposed hybrid-IC is simulated over static ISI and frequency selective fading channels. Simulations of the proposed scheme show that the performance is similar to the frequency-domain IC (FD-IC) but with lower complexity.

4. A detailed computational complexity analysis verifies that the operation of the $q$ filter in time domain is easier than in the frequency domain.

\subsection{Thesis Organization}

Chapter Two will describe the communication system model, including the transmitter, the channel and the receiver. It will also review frequency domain linear equalization.

Chapter Three describes the IC-based turbo equalization in the frequency domain. The coefficients of $P$-filter in the frequency domain and $q$-filter in the time domain for the proposed hybrid IC will be derived. At the end of the chapter, the computational complexity of LE and IC in the time and frequency domain, and the computational complexity of the time domain IC (TD-IC), FD-IC and hybrid IC will be compared. 
Chapter Four provides simulation results of the FD-IC and the hybrid IC over static ISI and frequency selective fading channels. This is accompanied by a discussion of the results and a comparison of the performance of the two schemes.

The conclusion will be summarized in Chapter Five along with a discussion on topics for possible future studies. 


\section{Chapter 2}

\section{SYSTEM MODEL AND REVIEW OF FREQUENCY DOMAIN LINEAR EQUALIZERS}

\subsection{Introduction}

In this chapter we present a simple channel model for transmission of coded data over an ISI channel, using a time domain linear equalizer to encounter the ISI. A brief overview of frequency domain linear equalization is also introduced. A block diagram of the communication system is shown in Figure 2-1. To improve the reliability of data transmission, a channel coding scheme is used, which adds controlled redundancy to the data. The coded data is fed into a transmit filter, which produces an analog baseband signal. The signal is then passed to a modulator to convert it to a desired frequency band using some modulation technique.

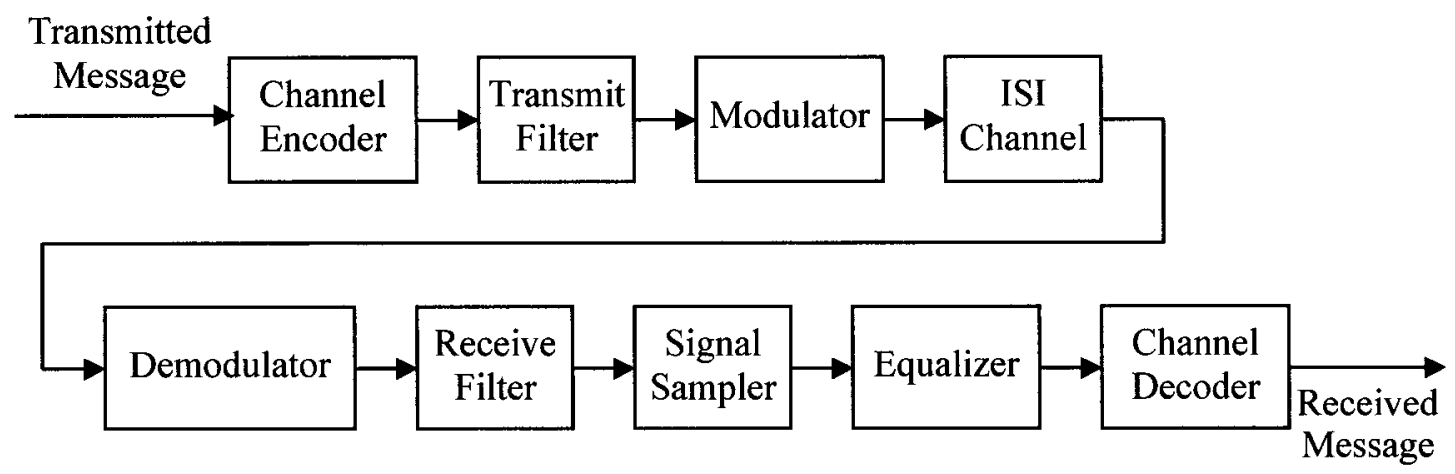

Figure 2-1. Block diagram of the communication system. 
The passband signal from the transmitter is then transmitted over the channel. For a wireless channel, important concerns are multipath propagation and frequency and time selective fading with time varying characteristics. Generally, the channel frequency response is not flat and will therefore cause ISI.

The received signal first goes to a coherent demodulator. The demodulated signal passes through the receive filter, which is matched to the transmit filter. The filtered signal is then sampled at the symbol rate to obtain the decision statistics for the symbol. These symbols are passed through an equalizer to remove the unwanted ISI while maximizing the signal-to-noise ratio. Finally, the equalized samples are fed into the channel decoder to estimate the transmitted message.

Mathematically, it is convenient to model the transmit filter, modulator, ISI channel, receive filter, demodulator and sampler as a discrete-time ISI channel. The transmission system then can be simplified as the composition of the transmitter, discrete-time ISI channel and receiver. These components are described in Sections 2.2, 2.3 and 2.4, respectively. The receiver discussed in Section 2.4 is based on using a time-domain linear equalizer. The use of a frequency domain linear equalizer is discussed in Section 2.5.

\subsection{Transmitter}

The transmitter is made up of a convolutional encoder, interleaver, and signal mapper, as shown in Figure 2-2.

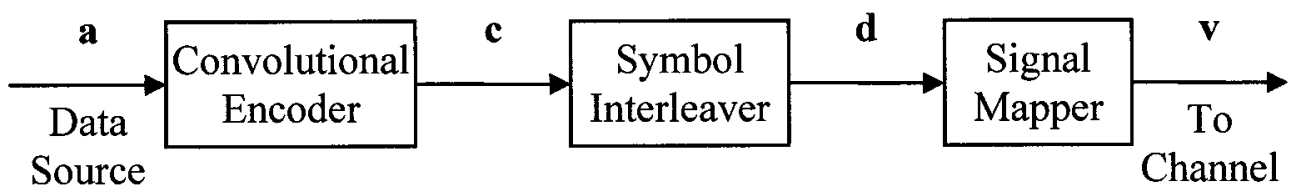

Figure 2-2. Structure of the transmitter. 


\subsubsection{Convolutional Encoder}

In the transmitter, a convolutional encoder is employed since the implementation of convolutional codes is well known and its soft-output decoding is useful for turbo equalization schemes. $N_{a}$ message symbols with $n_{a}$ message bits per symbol enter the encoder that produces $N_{c}$ code symbols with $n_{c}$ code bits per symbol. If the total memory of the encoder is $m$ bits then the number of states is $2^{m}$. The initial state is set to zero and to make the final state of encoder to be zero, a total of $\left(K_{c}-1\right)$ extra zero symbols are fed into the encoder after the message, where $K_{c}=m+1$ is the constraint length of the convolutional code. Therefore, the total codeword length is $N_{c}=N_{a}+K_{c}-1$ code symbols.

The message word can be expressed as $\mathbf{a}=\left[\begin{array}{llll}a_{0} & a_{1} & \ldots & a_{N_{a}-1}\end{array}\right]$, with each message symbol $a_{n} \in\left\{0,1, \cdots, 2^{n_{a}}-1\right\}$ for $0 \leq n \leq N_{a}-1$. It is assumed that the message symbols are all independent, and that each of the $2^{n_{a}}$ possible values for each symbol is equally likely to occur. That is, $\operatorname{Pr}\left\{a_{n}=a\right\}=\frac{1}{2^{n_{a}}}$, for all $a \in\left\{0,1, \ldots, 2^{n_{a}}-1\right\}$. The codeword can be expressed as $\mathbf{c}=\left[\begin{array}{lllll}c_{0} & c_{1} & \ldots & c_{N_{c}-1}\end{array}\right]$, with each code symbol $c_{n} \in\{0,1$, $\left.\ldots, 2^{n_{c}}-1\right\}$

\subsubsection{Interleaver}

The code symbols are fed into a random symbol interleaver, which rearranges the order of the code symbols, but keeps each symbol intact. The output of the interleaver has the same number of symbols and the same number of bits in each symbol compared with the input. The output of the interleaver is $\mathbf{d}=\left[\begin{array}{llll}d_{0} & d_{1} & \ldots & d_{N_{c}-1}\end{array}\right]$ with each symbol 
given by $d_{n}=c_{I L[n]}$, where $I L[\bullet]$ represents the interleaver mapping, which is a randomly selected permutation of the integers $\left\{0,1, \ldots, N_{c}-1\right\}$. Although randomly selected, both the transmitter and the receiver know the mapping.

\subsubsection{Symbol Mapper}

The interleaved symbols then go into the symbol mapper to be transformed to modulation symbols, which are points in a $M$-ary signal constellation, where $M=2^{n_{c}}$. The output of the symbol mapper is $\mathbf{v}=\left[\begin{array}{llll}v_{0} & v_{1} & \ldots & v_{N_{c}-1}\end{array}\right]$ with each modulation symbol given by $v_{n}=S M\left[d_{n}\right]$ where $S M\left[d_{n}\right]$ is the complex-valued point in the signal constellation corresponding to data symbol $d_{n}$.

The modulation symbols are used to modulate the carrier symbol for transmission over the ISI channel.

\subsection{Discrete-time ISI Channel Model}

The discrete-time ISI channel model incorporates the transmitter's pulse shaping filter and modulator, the analog ISI channel, and the receiver's demodulator, receive filter and symbol-rate signal sampler, as shown in Figure 2-3.

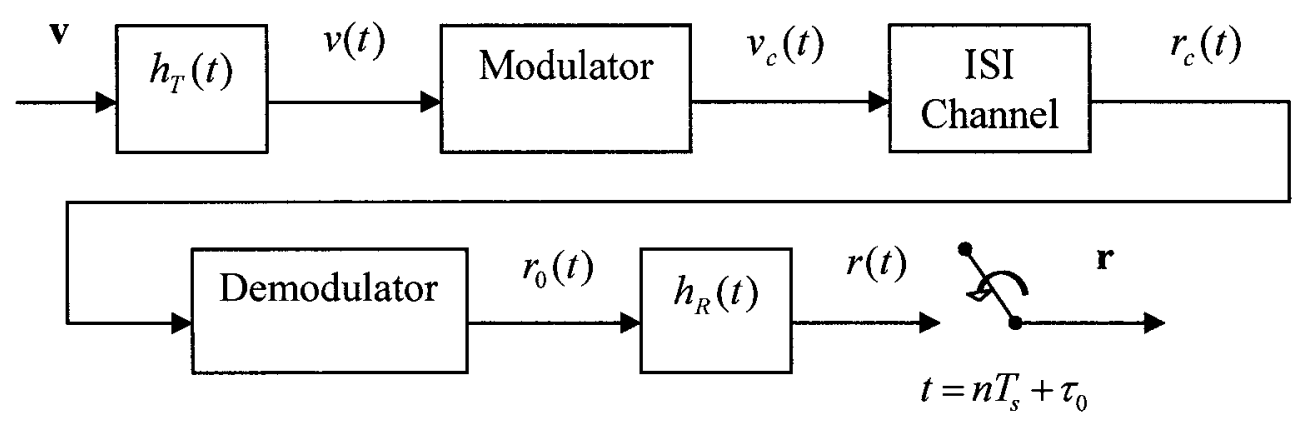

Figure 2-3. Discrete-time ISI channel model. 


\subsubsection{Pulse shaping filter}

To permit transmission over an analog channel, the complex equivalent lowpass signal

$$
v(t)=\sum_{n=0}^{N_{c}-1} v_{n} h_{T}\left(t-n T_{s}\right)
$$

is generated, where $h_{T}(t)$ is the desired pulse shape, and $T_{s}$ is the symbol period. To limit the spectral utilization of the transmitted signal, a band-limited pulse shape is often used, such as that produced by a root-raised-cosine filter, which has a frequency response of

$$
H_{T}(f)= \begin{cases}1, & \text { if }|f|<\frac{(1-\alpha)}{2 T_{s}} \\ \cos \left(\frac{T_{s}}{2 \alpha}\left[|f|-\frac{(1-\alpha)}{2 T_{s}}\right]\right), & \text { if } \frac{(1-\alpha)}{2 T_{s}} \leq|f| \leq \frac{(1+\alpha)}{2 T_{s}} \\ 0, & \text { if }|f|>\frac{(1+\alpha)}{2 T_{s}}\end{cases}
$$

where $\alpha$ is called the roll-off factor. It indicates how much bandwidth is being used over the ideal minimum bandwidth of $1 / T_{s}$.

\subsubsection{Modulator}

The baseband signal is transmitted over the physical channel with a carrier frequency of $f_{c}$, so the transmitted passband signal is

$$
v_{c}(t)=\operatorname{Re}\left\{v(t) \sqrt{2} e^{j 2 \pi f_{c} t}\right\}
$$

\subsubsection{Physical ISI channel}

The transmitted signal travels over multiple propagation paths to the receiver with different attenuation factors and propagation delays along each path. The received signal is modeled as 


$$
r_{c}(t)=\operatorname{Re}\left\{\sum_{i=1}^{N_{p}} \beta_{i} v\left(t-\tau_{i}\right) \sqrt{2} e^{j 2 \pi f_{c} t}\right\}+w_{c}(t),
$$

where $\beta_{i}$ represents the attenuation and phase rotation along path $i$, and $\tau_{i}$ is the corresponding propagation delay. $N_{p}$ is the number of propagation paths, and $w_{c}(t)$ represents the additive noise. For each path, $\beta_{i}$ is modeled as a complex Gaussian random variable, with zero mean and a variance of $\sigma_{i}^{2}=E\left[\left|\beta_{i}\right|^{2}\right]$. In this thesis we assume that perfect knowledge of $\beta_{i}$ and $\tau_{i}$ is available to the receiver as side information. Both $\sigma_{i}^{2}, \tau_{i}$ and $N_{p}$ are parameters of the specific channel model, some examples of which are given in Chapter 4. The noise, $w_{c}(t)$, is modeled as a zero mean stationary white Gaussian random process, with a single-sided power spectral density of $N_{0}$.

\subsubsection{Demodulator}

The demodulated signal is

$$
\begin{aligned}
r_{o}(t) & =r_{c}(t) \sqrt{2} e^{-j 2 \pi f_{c} t} \\
& =\frac{1}{2}\left(\sum_{i=1}^{N_{p}}\left[\beta_{i} v\left(t-\tau_{i}\right) \sqrt{2} e^{j 2 \pi f_{c} t}+\beta_{i}^{*} v^{*}\left(t-\tau_{i}\right) \sqrt{2} e^{-j 2 \pi f_{c} t}\right]\right) \sqrt{2} e^{-j 2 \pi f_{c} t} \\
& =\sum_{i=1}^{N_{p}} \beta_{i} v\left(t-\tau_{i}\right)+\sum_{i=1}^{N_{p}} \beta_{i}^{*} v^{*}\left(t-\tau_{i}\right) e^{-j 4 \pi f_{c} t}+w_{o}(t) \\
& =\sum_{i=1}^{N_{p}} \beta_{i} v\left(t-\tau_{i}\right)+\sum_{i=1}^{N_{p}} \beta_{i}^{*} v^{*}\left(t-\tau_{i}\right) e^{-j 4 \pi f_{c} t}+w_{o}(t)
\end{aligned}
$$

where $w_{o}(t)$ is the demodulated noise process. The first term in the right-hand side of the Equation (2-5) is the desired data-bearing signal, and the second is a high-frequency 
component that will be suppressed by the receive filter, therefore the second term in Equation (2-5) will not be considered.

\subsubsection{Receive filter}

In this communication system, the receive filter, with impulse response $h_{R}(t)$, is matched to the transmitted pulse shape, so that $h_{R}(t)=h_{T}^{*}(-t)$. The filtered received signal is

$$
\begin{aligned}
r(t) & =\int_{-\infty}^{+\infty} r_{o}(t-\tau) h_{R}(\tau) d \tau \\
& =\int_{-\infty}^{+\infty} \sum_{i=1}^{N_{p}} \beta_{i} v\left(t-\tau-\tau_{i}\right) h_{R}(\tau) d \tau+\int_{-\infty}^{+\infty} w_{0}(t-\tau) h_{R}(\tau) d \tau \\
& =\sum_{i=1}^{N_{p}} \beta_{i} \int_{-\infty}^{+\infty} v\left(t-\tau-\tau_{i}\right) h_{R}(\tau) d \tau+w(t) \\
& =\sum_{i=1}^{N_{p}} \beta_{i} \int_{-\infty}^{\infty} \sum_{n=0}^{N_{c}-1} v_{n} h_{T}\left(t-\tau-\tau_{i}-n T_{s}\right) h_{R}(\tau) d \tau+w(t) \\
& =\sum_{n=0}^{N_{c}-1} v_{n} \sum_{i=1}^{N_{p}} \beta_{i} h_{T R}\left(t-n T_{s}-\tau_{i}\right)+w(t),
\end{aligned}
$$

where $h_{T R}(t)=\int_{-\infty}^{+\infty} h_{T}(t-\tau) h_{R}(\tau) d \tau$ is the combined impulse response of the transmit and receive filters and $w(t)=\int_{-\infty}^{+\infty} w_{o}(t-\tau) h_{R}(\tau) d \tau$ is the filtered noise.

\subsubsection{Symbol-rate symbol sampler}

The received signal is sampled at the symbol rate, with a timing offset of $\tau_{0}=\min _{i} \tau_{i}$. The received samples are given by 


$$
\begin{aligned}
r_{n} & =r\left(n T_{s}+\tau_{0}\right) \\
& =\sum_{m=0}^{N_{c}-1} v_{m} \sum_{i=1}^{N_{p}} \beta_{i} h_{T R}\left(n T_{s}-m T_{s}-\tau_{i}+\tau_{0}\right)+w\left(n T_{s}+\tau_{0}\right) \\
& =\sum_{l=-\infty}^{+\infty} h_{l} v_{n-l}+w_{n},
\end{aligned}
$$

where

$$
h_{l}=\sum_{i=1}^{N_{p}} \beta_{i} h_{T R}\left(l T_{s}-\left(\tau_{i}-\tau_{0}\right)\right)
$$

are the equivalent discrete-time ISI channel coefficients, and $\left\{w_{n}\right\}$ are the noise samples, which are independent complex Gaussian random variables with zero mean and $E\left[\left|w_{n}\right|^{2}\right]=N_{0}$ if $h_{R}(t)$ is such that $\int_{-\infty}^{+\infty}\left|h_{R}(t)\right|^{2} d t=1$

In practice, the channel delay spread is finite, and $h_{T R}(t)$ is typically truncated to some finite duration. As such, we can assume that $h_{l}$ is non-zero only for $l \in\{0,1, \ldots$, $L\}$, for some $L$. Therefore Equation (2-7) can be rewritten as

$$
r_{n}=\sum_{l=0}^{L} h_{l} v_{n-l}+w_{n}
$$

It is clear from Equation (2-9) that each transmitted symbol affect $L+1$ different received samples, and that each received sample depends on $L+1$ different transmitted symbols. Intersymbol interference in therefore present. The received samples are passed to rest of the receiver for further processing. 


\subsection{Receiver}

The receiver is made up of an equalizer, a deinterleaver and a decoder as shown in Figure 2-4.

\subsubsection{Equalizer}

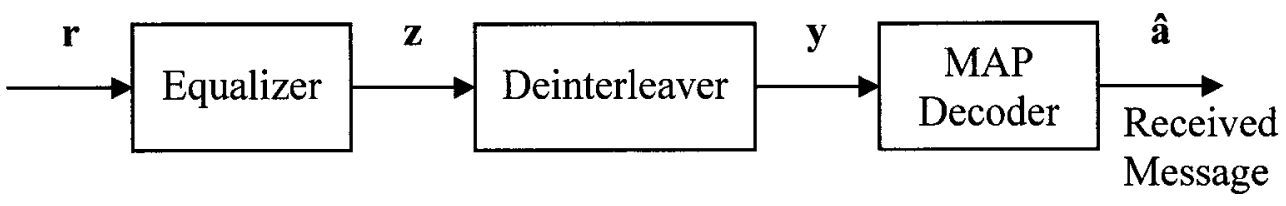

Figure 2-4. Structure of the receiver.

To remove the ISI, several different methods of equalization have been proposed, such as LE, DFE, MLSE and MAP equalization. Both MLSE and MAP equalization are optimal techniques but have high complexity, so LE and DFE are generally used in practice. In this thesis we study turbo equalization, and since it has been shown that LE generally gives better performance than DFE when used with turbo equalization [23], with lower complexity, only LE is considered in this thesis.

The linear equalizer employs a transversal filter. Although in theory it is necessary to use an infinite number of coefficients to remove all the ISI, in practice the number of coefficients are generally chosen to be finite, and depends on the length of channel impulse response.

For a transversal filter with $2 K+1$ taps, the equalizer output is

$$
z_{n}=\sum_{k=-K}^{+K} p_{k} r_{n-k}
$$

where $\left\{p_{k}\right\}$ are the filter coefficients (tap weights). The filter coefficients are typically chosen according to the minimum mean square error (MMSE) criterion. That is, $\left\{p_{k}\right\}$ 
are chosen to minimize the mean of the square of the error between the equalizer output and the corresponding transmitted symbol, $E\left[\left|z_{n}-v_{n}\right|^{2}\right]$. Under this criterion the filter coefficients are given as the solutions to the $2 K+1$ equations [8].

$$
\sum_{k=-K}^{K} p_{k}\left[\sigma_{v}^{2} \sum_{a=0}^{L} h_{a}^{*} h_{l-k+a}+N_{0} \delta_{l-k}\right]=\sigma_{v}^{2} h_{-l}^{*}
$$

for $l \in\{-K,-K+1, \ldots, K-1, K\}$, where $\delta$. is the Kronecker delta, and $\sigma_{v}^{2}=E\left[\left|v_{n}\right|^{2}\right]$ is the average transmitted energy per symbol. In Equation (2-11) we define $h_{l}=0$ for $l \notin\{0,1, \ldots, L\}$.

\subsubsection{Deinterleaver}

The equalized samples $\mathbf{z}=\left[\begin{array}{lllll}z_{0} & z_{1} & \ldots & z_{N_{c}-1}\end{array}\right]$ are fed into a symbol deinterleaver, which rearranges the order of the samples so that they are in the same order as the original code symbols. The output of the deinterleaver is $\mathbf{y}=\left[\begin{array}{llll}y_{0} & y_{1} \ldots & y_{N_{c}-1}\end{array}\right]$, with each sample given by $y_{n}=z_{I L^{-1}[n]}$ where $I L^{-1}[\bullet]$ represents the deinterleaver mapping, which is the inverse permutation of the selected permutation used in the transmitter (that is $I L^{-1}[I L(n)]=n$ ).

\subsubsection{MAP Decoder}

The convolutional code is decoded using a maximum a posterori probability (MAP) decoder, which is based on minimizing the probability of a message bit error. The decoder calculates the a posterori probabilities (APP's) $\operatorname{Pr}\left\{a_{n}=a \mid \mathbf{y}\right\}$ for all $n \in\left\{0,1, \ldots, N_{a}-1\right\}$ and $a \in\left\{0,1, \ldots, 2^{n_{a}}-1\right\}$. The decoder output for each message symbol is the possible value of $a$ that maximizes the APP's. That is 


$$
\hat{a}_{n}=\arg \max _{a} \operatorname{Pr}\left\{a_{n}=a \mid \mathbf{y}\right\} .
$$

Calculation of the APP's is performed efficiently using the APP algorithm by Chang \& Hancock [24], which is described in the context of decoding convolutional codes by Bahl et al. [25].

Implementation of this algorithm involves the use of branch metrics, which are the likelihood functions for each deinterleaved equalizer output symbol for each of the possible transmitted code symbols, $\gamma_{n}(c)=f\left(y_{n} \mid c_{n}=c\right)$, where $y_{n}$ is the output of the deinterleaver after equalizer. Taking into account the symbol interleaving at the transmitter, and the deinterleaver at the receiver, we have

$$
\gamma_{n}(c)=f\left(z_{I L^{-1}[n]} \mid d_{I L^{-1}[n]}=c\right)=f\left(z_{I L^{-1}[n]} \mid v_{I L^{-1}[n]}=S M[c]\right)
$$

To calculate the branch metrics we therefore need an expression for $f\left(z_{n} \mid v_{n}=v\right)$. Under the typical assumption that the residual ISI after equalization has a Gaussian distribution, we have

$$
f\left(z_{n} \mid v_{n}=v\right)=\frac{1}{\pi \sigma_{z}^{2}} \exp \left\{-\frac{1}{\sigma_{z}^{2}}\left|z_{n}-\mu_{z}(v)\right|^{2}\right\}
$$

where the mean is

$$
\mu_{z}(v)=E\left[z_{n} \mid v_{n}=v\right]
$$

and the variance is

$$
\sigma_{z}^{2}=E\left[\left|z_{n}-\mu_{z}(v)\right|^{2} \mid v_{n}=v\right] .
$$

For the linear equalizer described in Section 2.4.1, we have

$$
\mu_{z}(v)=v \sum_{k=-K}^{K} p_{k} h_{-k}
$$

and 


$$
\sigma_{z}^{2}=\sigma_{v}^{2}\left(\sum_{k=-K}^{K} p_{k} h_{-k}\right)^{*}\left(1-\sum_{k=-K}^{K} p_{k} h_{-k}\right)
$$

where $\sigma_{v}^{2}=E\left[\left|v_{n}\right|^{2}\right]$ is the average transmitted energy per symbol.

\subsection{Frequency Domain Linear Equalization}

For channels with long delay spread, the complexity of the time-domain linear equalizer can be quite large since a large number of filter taps are required (the number of taps must be several times greater than the length of the channel impulse response). In such situations it was shown by Falconer et al. that complexity can be reduced by implementing the equalizer in the frequency domain [29].

A block diagram of a frequency domain linear equalizer (FD-LE) is shown in Figure 2.5. The received samples, $r_{n}$ are converted to the frequency domain with a fast Fourier transform (FFT). The frequency domain samples $R_{k}$ are multiplied by the filter coefficients $P_{k}$, giving $Z_{k}$. These are transformed back to the time domain using an inverse FFT, giving the equalizer output samples $z_{n}$. The optimal filter coefficients are found according the MMSE criterion, and are given by [29]

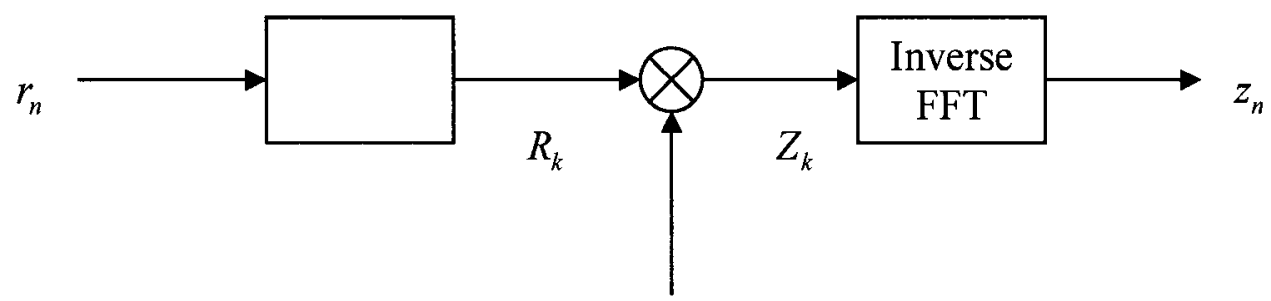

Figure 2-5. Frequency domain linear equalizer. 


$$
P_{k}=\frac{\sigma_{v}^{2} H_{k}^{*}}{\sigma_{v}^{2}\left|H_{k}\right|^{2}+N_{0}} \quad \forall k \in\{0,1, \ldots, N-1\},
$$

where

$$
H_{k}=\sum_{n=0}^{N-1} h_{n} e^{-j 2 \pi k n / N}
$$

are the Discrete Fourier Transform (DFT) of the discrete-time ISI channel impulse response, and $N=N_{c}$ is the DFT block length. In this thesis, it is assumed that a radix2 FFT is used to compute the DFT. These coefficients are only optimal under the assumption that the ISI results from a circular convolution of the transmitted data symbols with the channel impulse response, instead of a linear convolution as is actually the case. To support this assumption it is necessary for the transmitter to place a "cyclic-prefix" at the beginning of each block, whereby the last $L$ symbols of the block, $\left[\begin{array}{llll}v_{N-L} & v_{N-L+1} & \ldots & v_{N-1}\end{array}\right]$, are duplicated and transmitted immediately prior to the transmission of the block. By doing so, the linear convolution of the channel is equivalent to a circular convolution.

After the inverse FFT the equalized samples are deinterleaved and passed to the MAP decoder. This processing is done in the same manner as for the time-domain linear equalization, except that when calculating the branch metrics according to Equation (2-14), the mean and variance are given by (as shown in Appendix A),

$$
\mu_{z}(v)=v q_{0}
$$

and

$$
\sigma_{z}^{2}=\sigma_{v}^{2} q_{0}^{*}\left(1-q_{0}\right)
$$

with $q_{0}=\frac{1}{N} \sum_{k=0}^{N-1} P_{k} H_{k}$. 


\section{Chapter 3}

\section{FREQUENCY DOMAIN TURBO EQUALIZATION}

\subsection{Introduction to Turbo Equalization}

The receiver structures presented in Sections 2.4 and 2.5 are inherently suboptimal because they treat the equalization and decoding as two separate tasks. An optimal receiver requires that the ISI and channel coding be treated as a single code, jointly decoded. When interleaving is used, such an optimal receiver has an enormous complexity.

Motivated by the discovery of turbo codes in 1993 [1], turbo equalization was proposed in 1995 by Douillard et al. [7]. Based on the use of a MAP equalizer and a MAP decoder through an iterative decoding process, its performance has proved to be near optimum in eliminating the effect of ISI.

However, since the complexity of the MAP equalizer is still very high, particularly for channels with long delay spreads, the application of this structure is limited. As a consequence, it is necessary to find more practical alternative methods to reduce the prohibitive complexity. An approach based on an interference canceller instead of the MAP equalizer was proposed in 1997 by Glavieux et al. [17], and shown to have significantly reduced complexity. In this thesis, IC-based turbo equalization is studied further. 


\subsubsection{Interference Canceller}

A block diagram of an IC is shown in Figure 3-1, and the output is given by

$$
z_{n}=\sum_{k=-L}^{0} p_{k} r_{n-k}-\sum_{\substack{k=-L \\ k \neq 0}}^{L} q_{k} v_{n-k}
$$

where the filter coefficients, $\left\{p_{k}\right\}$ and $\left\{q_{k}\right\}$, are determined according to the MMSE criterion to be [17]

$$
p_{k}=\frac{\sigma_{v}^{2} h_{-k}^{*}}{\sigma_{v}^{2} E_{h}+N_{0}}
$$

and

$$
q_{k}=\sum_{l=-L}^{0} p_{l} h_{k-l}
$$

where $E_{h}=\sum_{l=0}^{L}\left|h_{l}\right|^{2}$ is the overall channel attenuation.

By substituting Equation (2-9) for $r_{n-k}$ in Equation (3-1), it is clear that

$$
z_{n}=\frac{\sigma_{v}^{2} E_{h}}{\sigma_{v}^{2} E_{h}+N_{0}} v_{n}+\sum_{k=-L}^{0} p_{k} w_{n-k},
$$

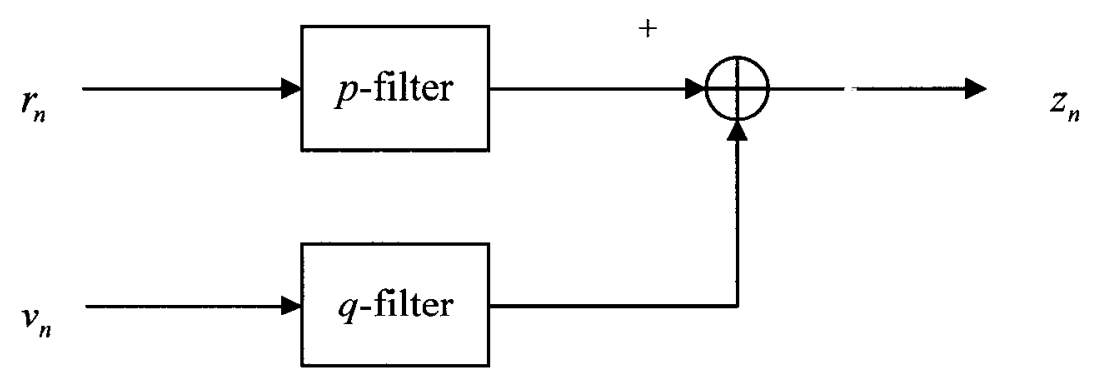

Figure 3-1. Block diagram of an interference canceller. 
so the ISI is completely removed. Of course, use of Equation (3-1) is only possible if $v_{n-L}, \cdots, v_{n-1}, v_{n+1}, \cdots, v_{n+L}$ are known, which is not the case in practice. Fortunately, this problem can be addressed by using turbo equalization.

\subsubsection{IC-based turbo equalization}

A block diagram of an IC-based turbo equalizer is shown in Figure 3-2. The received samples are first processed using a linear equalizer, as described in Section 2.4.1. The equalizer output samples are deinterleaved and used as input to the MAP decoder which decodes the convolutional code. This processing is the same as described in Section 2.4. However, the MAP decoder is modified slightly to produce not only the a posteriori probabilities (APP's) of the message symbols, but also the APP's of the code symbols,

$$
\varphi_{n}(c)=\operatorname{Pr}\left\{c_{n}=c \mid \mathbf{y}\right\}
$$

for $n \in\left\{0,1, \cdots, N_{c}-1\right\}$ and $c \in\left\{0,1, \cdots, 2^{n_{c}}-1\right\}$.

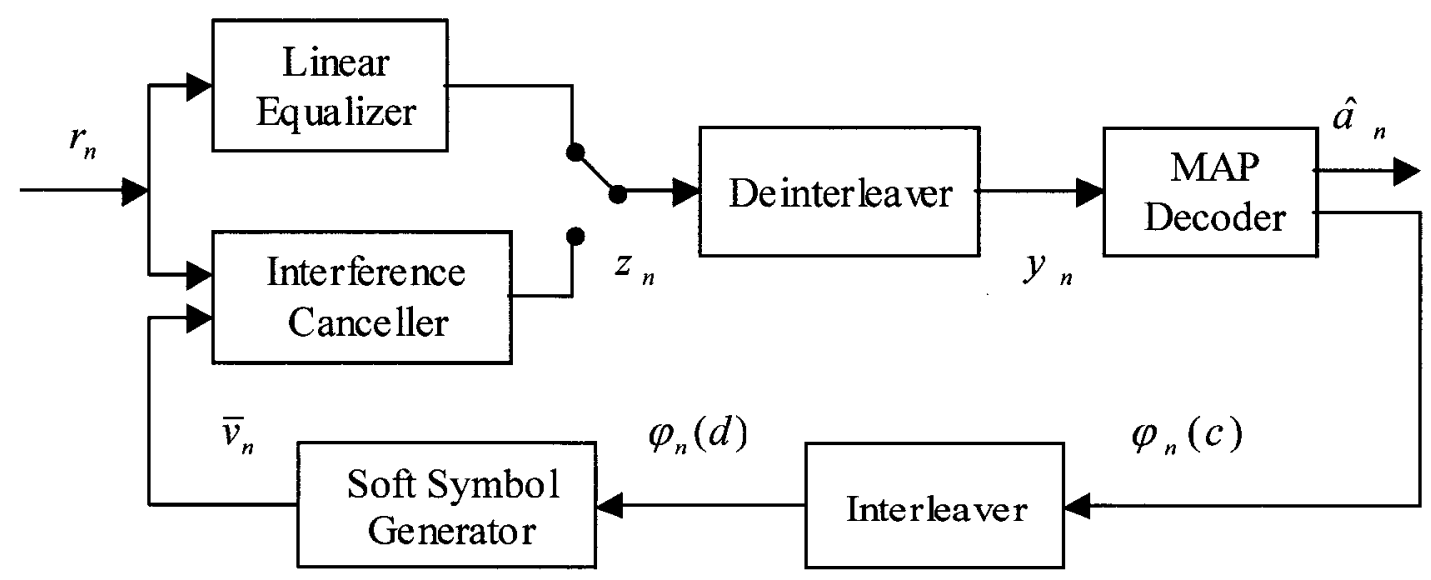

Figure 3-2. Block diagram of IC-based turbo equalizer. 
The code symbol APP's are interleaved using the same permutation selected at the transmitter, giving

$$
\phi_{n}(d)=\varphi_{I L[n]}(d)
$$

for $d \in\left\{0,1, \cdots, 2^{n_{c}}-1\right\}$. The interleaved APP's are then used to generate soft symbols according to

$$
\bar{v}_{n}=\sum_{d=0}^{M-1} S M[d] \phi_{n}(d)
$$

These soft symbols are estimates of the transmitted symbols, generated at the receiver based on knowledge of the transmitted data as determined by the MAP decoder.

By using the soft symbol $\bar{v}_{n-k}$ in place of $v_{n-k}$ in Equation (3-1), the interference canceller can be implemented. Although the transmitted symbols are not known, if the soft symbols are reasonably accurate estimates, the IC will be reasonably effective at removing the ISI.

The IC is applied to the received data, and the IC output, $\left\{z_{n}\right\}$, is deinterleaved and passed to the MAP decoder. When calculating the branch metrics based on the IC output, Equation (2-14) is used, with the sample mean and variance given by (as shown in Appendix A)

$$
\mu_{z}(v)=v q_{0}
$$

and

$$
\sigma_{z}^{2}=v q_{0}^{*}\left(1-q_{0}\right)
$$

where $q_{0}=\frac{\sigma_{v}^{2} E_{h}}{\sigma_{v}^{2} E_{h}+N_{0}}$. 
The MAP decoder output, $\varphi_{n}(c)$, could again be interleaved and fed back to the IC, and the process could be repeated in an iterative fashion. With each iteration the soft symbols become more accurate estimates of $v_{n}$, so the IC become more effective at eliminating the ISI. Turbo equalization is therefore a very useful technique for processing signals corrupted by ISI [17].

\subsection{Turbo Equalization Using Frequency Domain IC}

From Equation (3-1) the output of the time-domain IC is

$$
\begin{aligned}
z_{n} & =\sum_{k=-L}^{0} p_{k} r_{n-k}-\sum_{\substack{k=-L \\
k \neq 0}}^{L} q_{k} v_{n-k} \\
& =\sum_{k=-L}^{0} p_{k} r_{n-k}-\sum_{k=-L}^{L} q_{k} v_{n-k}+q_{0} v_{n} .
\end{aligned}
$$

If the two filters were implemented in the frequency domain, the output of the IC would be of the form

$$
z_{n}=\frac{1}{N} \sum_{k=0}^{N-1}\left(P_{k} R_{k}-Q_{k} V_{k}\right) e^{j 2 \pi k n / N}+q_{0} v_{n}
$$

where $\left\{R_{k}\right\}$ and $\left\{V_{k}\right\}$ are the DFT's of $\left\{r_{n}\right\}$ and $\left\{v_{n}\right\}$, respectively, and $N=N_{c}$ is the DFT block length. By applying the MMSE criterion, it is shown in Appendix A that the optimal filter coefficients are

$$
\begin{aligned}
& P_{k}=\frac{\sigma_{v}^{2} H_{k}^{*}}{\sigma_{v}^{2} E_{h}+N_{0}}, \\
& Q_{k}=P_{k} H_{k}=\frac{\sigma_{v}^{2}\left|H_{k}\right|^{2}}{\sigma_{v}^{2} E_{h}+N_{0}},
\end{aligned}
$$

and 


$$
q_{0}=\frac{\sigma_{v}^{2} E_{h}}{\sigma_{v}^{2} E_{h}+N_{0}}
$$

where $H_{k}=\sum_{n=0}^{N-1} h_{n} e^{-j 2 \pi k n / N}$ is the DFT of the channel coefficients.

Equation (3-11) can be manipulated slightly to be

$$
\begin{aligned}
z_{n} & =\frac{1}{N} \sum_{k=0}^{N-1}\left(P_{k} R_{k}-Q_{k} V_{k}\right) e^{j 2 \pi k n / N}+\frac{1}{N} \sum_{k=0}^{N-1} q_{0} V_{k} e^{j 2 \pi k n / N} \\
& =\frac{1}{N} \sum_{k=0}^{N-1}\left(P_{k} R_{k}-\left(Q_{k}-q_{0}\right) V_{k}\right) e^{j 2 \pi k n / N} \\
& =\frac{1}{N} \sum_{k=0}^{N-1}\left(P_{k} R_{k}-\widetilde{Q}_{k} V_{k}\right) e^{j 2 \pi k n / N}
\end{aligned}
$$

where

$$
\widetilde{Q}_{k}=Q_{k}-q_{0}=\frac{\sigma_{v}^{2}\left|H_{k}\right|^{2}}{\sigma_{v}^{2} E_{h}+N_{0}}-\frac{\sigma_{v}^{2} E_{h}}{\sigma_{v}^{2} E_{h}+N_{0}}=\frac{\sigma_{v}^{2}\left(\left|H_{k}\right|^{2}-E_{h}\right)}{\sigma_{v}^{2} E_{h}+N_{0}} .
$$

Turbo equalization is performed as described in Section 3.1, except that the interference canceller shown in Figure 3-2 is implemented in the frequency domain as in Figure 3-3.

When calculating the branch metrics according to Equation (2.14), the MCU uses (as shown in Appendix A), a mean of

$$
\mu_{z}(v)=q_{0} v
$$

and a variance of

$$
\begin{aligned}
& \sigma_{z}^{2}=\sigma_{v}^{2} q_{0}^{*}\left(1-q_{0}\right), \\
& \text { where } q_{0}=\frac{\sigma_{v}^{2} E_{h}}{\sigma_{v}^{2} E_{h}+N_{0}} .
\end{aligned}
$$




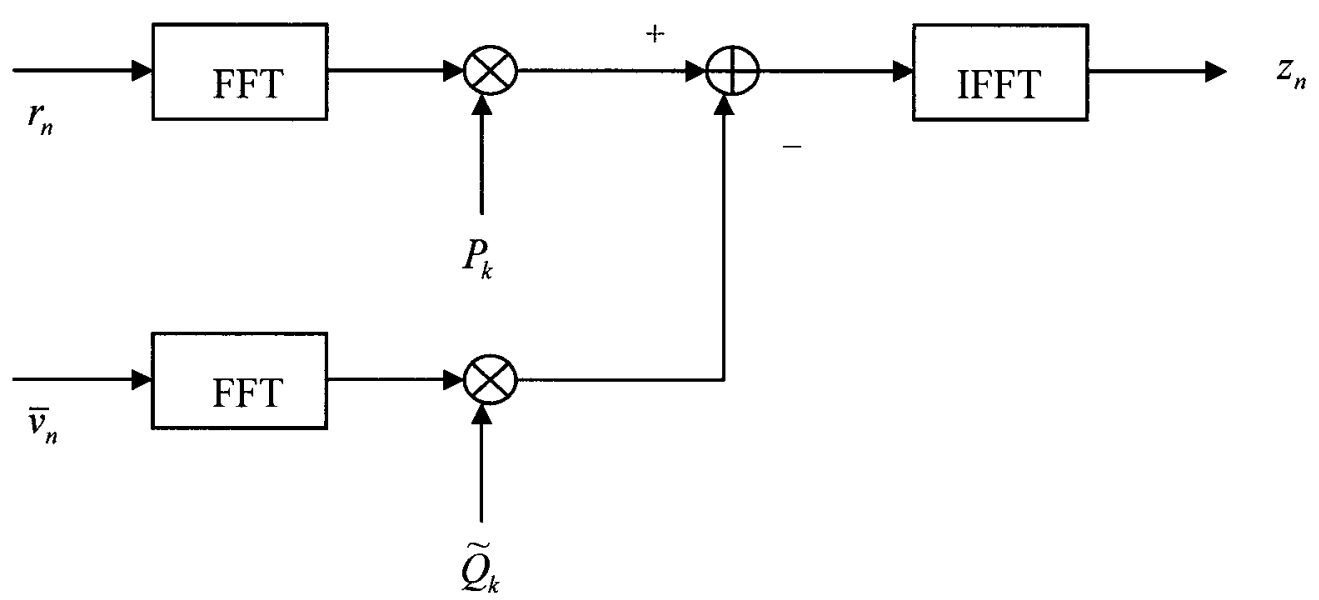

Figure 3-3. Structure of the frequency domain IC.

\subsection{Reduced Complexity Hybrid Frequency/Time Domain IC}

\subsubsection{Motivation}

As will be discussed in Section 3.4, implementation of the IC in the frequency domain instead of the time domain leads to a substantial reduction in the system complexity. However, careful examination of Figures 3-2 and 3-3 reveals that one FFT and one IFFT operation are required for each iteration of the turbo equalizer. Since FFT's are not trivial operations, it is desirable to avoid their use within the iterative loop. Let us review the coefficients of the $q$-filter in the time domain. Observing Equation (3-3), if most of values of $h_{k}$ are zero, then most of the $q_{k}$ coefficients will be zero. Similarly, if most of values of $h_{k}$ are small, then most of the $q_{k}$ coefficients will be near to zero. In other words, the energy of some taps will be much greater than the total energy of the rest of the taps. In this situation, the system can be simplified so that 


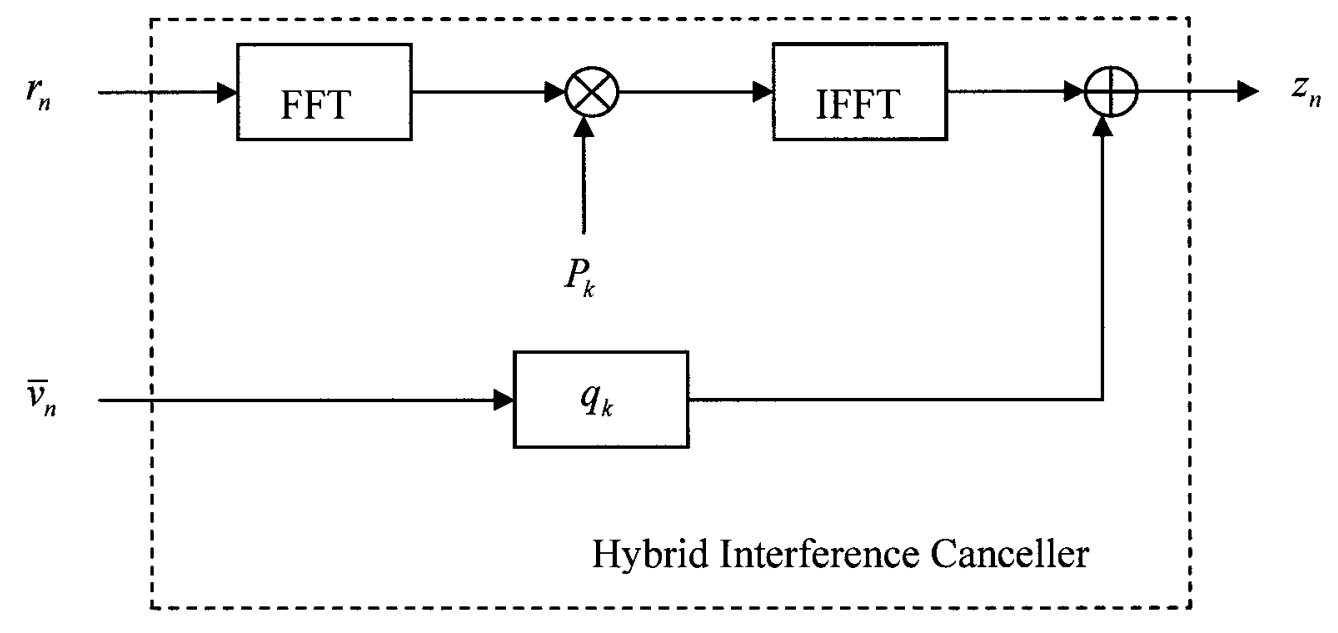

Figure 3-4. Structure of the hybrid IC.

only the few largest $q_{k}$ are applied and the rest of the $q_{k}$ are ignored. In such case the $q$-filter can be implemented in the time domain with reasonable complexity.

Therefore, the proposed hybrid frequency /time domain IC shown in Figure 3-4 is introduced. The $P$-filter still operates in the frequency domain but the $q$-filter operates in the time domain. In the proposed model, the $P$-filter and $q$-filter will not have the same coefficients as presented in Equation (3-12) and (3-3). These coefficients will depend on how many $q$-filter taps are chosen to use. If the numbers of taps are chosen equal to the channel delay spread, the $P$-filter and $q$-filter will have the same values as (3-12) and (3-3), respectively.

\subsubsection{Coefficients of the hybrid IC}

The hybrid IC is made up of two parts, the $P$-filter operating in frequency domain and the $q$-filter operating in the time domain. The output of the IC is

$$
z_{n}=\frac{1}{N} \sum_{k=0}^{N-1} P_{k} R_{k} e^{j 2 \pi k n / N}-\sum_{i=1}^{N_{t}}\left(q_{M_{i}} v_{n-M_{i}}+q_{-M_{i}} v_{n+M_{i}}\right) \text {, }
$$


where $N_{t}$ is the number of feedback tap pairs and $\left\{M_{i} \mid i \in\left\{1,2, \cdots N_{t}\right\}\right\}$ are the indices of the desired feedback taps. The system performance will depend on $N_{t}$, in that larger $N_{t}$ should provide better performance, but with higher computational complexity. Typically $N_{t}$ is less than or equal to 3 . The $N_{t}$ largest values of $q_{k}$ and their positions can be found using Equation (3-3). These chosen $q_{k}$ are rearranged in ascending order according to the values and give them to $q_{M_{i}}$ for $\left\{M_{i} \mid i \in\left\{1,2, \cdots N_{t}\right\}\right\}$.

Using the mean square error criterion to get the optimum coefficients, it is shown in Appendix B that

$$
P_{l}=\frac{H_{l}^{*} \sigma_{v}^{2}}{\left|H_{l}\right|^{2} \sigma_{v}^{2}+N_{0}}\left[1+\sum_{i=1}^{N_{t}}\left(q_{M_{i}} e^{-j 2 \pi M_{i} l / N}+q_{-M_{i}} e^{j 2 \pi M_{i} l / N}\right)\right]
$$

and

$$
q_{m}=\frac{1}{N} \sum_{k=0}^{N-1} P_{k} H_{k} e^{j 2 \pi k m / N}
$$

for $l \in\{0,1, \cdots, N-1\}$ and $m \in\left\{M_{1}, M_{2}, \cdots, M_{N_{t}}\right\}$. Substituting Equation (3-20) into (321) gives

$$
\begin{aligned}
q_{m} & =\frac{1}{N} \sum_{k=0}^{N-1} \frac{H_{k}^{*} \sigma_{v}^{2}}{\left|H_{k}\right|^{2} \sigma_{v}^{2}+N_{0}}\left[1+\sum_{i=1}^{N_{t}}\left(q_{M_{i}} e^{-j 2 \pi M_{i} k / N}+q_{-M_{i}} e^{j 2 \pi M_{i} k / N}\right)\right] H_{k} e^{j 2 \pi n k / N} \\
& =x_{m}+\sum_{i=1}^{N_{t}}\left(q_{M_{i}} x_{m-M_{i}}+q_{-M_{i}} x_{m+M_{i}}\right)
\end{aligned}
$$

where

$$
x_{m}=\frac{1}{N} \sum_{k=0}^{N-1} \frac{\left|H_{k}\right|^{2} \sigma_{v}^{2}}{\left|H_{k}\right|^{2} \sigma_{v}^{2}+N_{0}} e^{j 2 \pi m k / N}
$$

is the inverse DFT of 


$$
X_{k}=\frac{\left|H_{k}\right|^{2} \sigma_{v}^{2}}{\left|H_{k}\right|^{2} \sigma_{v}^{2}+N_{0}} \text {. }
$$

By defining

$$
\begin{aligned}
& \mathbf{q}=\left[q_{M_{1}} \cdots q_{M_{N_{t}}} q_{-M_{1}} \cdots q_{-M_{N_{t}}}\right]^{T}, \\
& \mathbf{x}=\left[x_{M_{1}} \cdots x_{M_{N_{t}}} x_{-M_{1}} \cdots x_{-M_{N_{t}}}\right]^{T},
\end{aligned}
$$

and

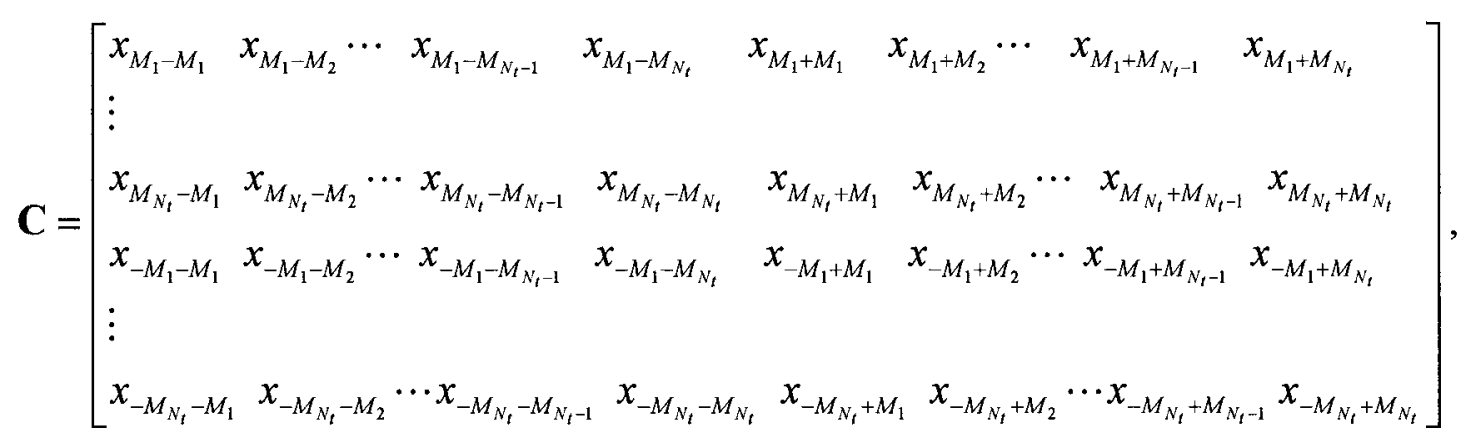

Equation (3-22) can be written as

$$
\mathbf{q}=\mathbf{x}+\mathbf{C q},
$$

To simplify the calculation of $\mathbf{C}, \mathbf{C}$ can be expressed as

$$
\mathbf{C}=\left[\begin{array}{cc}
\mathbf{F} & \mathbf{E} \\
\mathbf{E}^{*} & \mathbf{F}^{*}
\end{array}\right],
$$

where

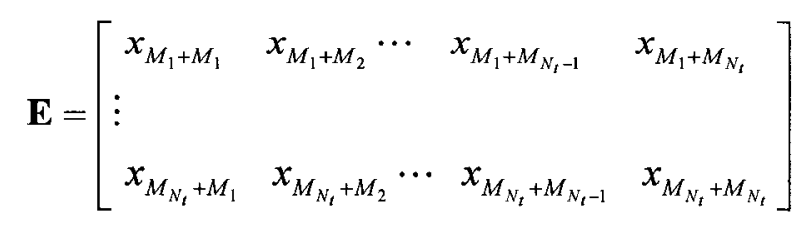

and 


$$
\mathbf{F}=\left[\begin{array}{lllll}
x_{M_{1}-M_{1}} & x_{M_{1}-M_{2}} \cdots & x_{M_{1}-M_{N_{t}-1}} & x_{M_{1}-M_{N_{t}}} \\
\vdots & & & & \\
x_{M_{N_{t}}-M_{1}} & x_{M_{N_{t}}-M_{2}} \cdots & x_{M_{N_{t}}-M_{N_{t}-1}} & x_{M_{N_{t}}-M_{N_{t}}}
\end{array}\right]
$$

By defining $\quad \mathbf{q}_{1}=\left[q_{M_{1}} \cdots q_{M_{N_{t}}}\right]^{T}, \mathbf{q}_{2}=\left[q_{-_{1}} \cdots q_{-M_{N_{t}}}\right]^{T}, \mathbf{x}_{1}=\left[x_{M_{1}} \cdots x_{M_{N_{t}}}\right]^{T} \quad$ and $\mathbf{x}_{2}=\left[x_{-M_{1}} \cdots x_{-M_{N_{t}}}\right]^{T}$, then Equation (3-28) can be rewritten as

$$
\left[\begin{array}{cc}
\mathbf{I}-\mathbf{F} & -\mathbf{E} \\
-\mathbf{E}^{*} & \mathbf{I}-\mathbf{F}^{*}
\end{array}\right]\left[\begin{array}{l}
\mathbf{q}_{1} \\
\mathbf{q}_{2}
\end{array}\right]=\left[\begin{array}{l}
\mathbf{x}_{1} \\
\mathbf{x}_{2}
\end{array}\right]
$$

From Equation (3-32), we obtain

$$
\mathbf{q}_{1}=\left[(\mathbf{I}-\mathbf{F})-\mathbf{E}\left(\mathbf{I}-\mathbf{F}^{*}\right)^{-1} \mathbf{E}^{*}\right]^{-1}\left[\mathbf{x}_{1}+\mathbf{E}\left(\mathbf{I}-\mathbf{F}^{*}\right)^{-1} \mathbf{x}_{2}\right]
$$

and

$$
\mathbf{q}_{2}=\left[\left(\mathbf{I}-\mathbf{F}^{*}\right)-\mathbf{E}^{*}(\mathbf{I}-\mathbf{F})^{-1} \mathbf{E}\right]^{-1}\left[\mathbf{E}^{*}(\mathbf{I}-\mathbf{F})^{-1} \mathbf{x}_{1}+\mathbf{x}_{2}\right]
$$

Since it can be proved that $q_{-m}=q_{m}^{*}$ according to Equation (3-21), actually, calculating either $\mathbf{q}_{1}$ or $\mathbf{q}_{2}$ is sufficient. The P-filter coefficients can be calculated according to Equation (3-20).

\subsubsection{Mean and Variance of the IC output}

When calculating the branch metrics according to Equation (2-14), the MCU uses (as shown in Appendix B), a mean of

$$
\mu_{z}(v)=q_{0} v
$$

and a variance of

$$
\sigma_{z}^{2}=\sigma_{v}^{2} q_{0}^{*}\left(1-q_{0}\right)
$$

where $q_{0}$ is given by Equation (3-21). 


\subsection{Complexity Comparison}

In this section we analyze the complexity of various components of the equalizer, including the time-domain linear equalizer (TD-LE), frequency domain linear equalizer (FD-LE), time-domain IC (TD-IC), frequency-domain IC (FD-IC) and hybrid IC, in terms of the number of multiplications and additions. In this thesis, calculating complexities for the LE, the FD-IC and the hybrid reduced complexity IC is based on the assumption that a radix-2 FFT is used to compute the DFT. The application of FFT is a consideration of its speed advantage over DFT. The number of complex multiplications in a direct DFT implementation is of the order of $N^{2}$, where $N$ is the DFT block length. If $N$ is an integer power of 2 and a radix-2 FFT is used, then only $\frac{N}{2} \log _{2} N$ complex multiplications are needed. If only considering the multiplication factor, it is obvious that the speed advantage is approximately:

$$
\frac{N^{2}}{\frac{N}{2} \log _{2} N}=\frac{2 N}{\log _{2} N}
$$

As the transform length increases, the advantage will be more obvious. For instance, when $N=16$, the ratio is 8 ; when $N=1024$, the ratio will be approximately 205 .

One FFT needs $\frac{1}{2} \log _{2} N$ complex multiplications and $\log _{2} N$ additions per symbol. One complex multiplication requires four real multiplications and two additions. The numbers of real multiplications and real additions for one FFT is shown in Table 3-1, where $C M, C A, R M$ and $R A$ stand for complex multiplication, complex addition, real multiplication and real addition, respectively. 


\begin{tabular}{|c|c|c|}
\hline & $R M$ & $R A$ \\
\hline$\frac{1}{2} \log _{2} N \times C M$ & $2 \log _{2} N$ & $\log _{2} N$ \\
\hline $\log _{2} N \times C A$ & & $2 \log _{2} N$ \\
\hline total per FFT & $2 \log _{2} N$ & $3 \log _{2} N$ \\
\hline
\end{tabular}

Table 3-1. Complexity per symbol of one FFT.

\begin{tabular}{|c|c|c|}
\hline & $R M$ & $R A$ \\
\hline$(2 K+1) \times C M$ & $4(2 K+1)$ & $2(2 K+1)$ \\
\hline$(2 K) \times C A$ & & $2(2 K)$ \\
\hline total & $8 K+4(=32 L+4)$ & $8 K+2(=32 L+2)$ \\
\hline
\end{tabular}

Table 3-2. Complexity per symbol of the TD-LE.

\begin{tabular}{|c|c|c|}
\hline & $R M$ & $R A$ \\
\hline $2 \times \mathrm{FFT}$ & $4 \log _{2} N$ & $6 \log _{2} N$ \\
\hline $1 \times C M$ & 4 & 2 \\
\hline total & $4 \log _{2} N+4$ & $6 \log _{2} N+2$ \\
\hline
\end{tabular}

Table 3-3. Complexity per symbol of the FD-LE. 


\subsubsection{Complexity of LE}

In the time domain, we assume that the total number of taps of the LE is $2 K+1$, with $K=4 L$, typically, where $\mathrm{L}$ is channel impulse response length. The complexity is shown in Table 3-2. In the frequency domain one FFT and one IFFT are needed, as well as one complex multiplication per symbol to apply the filter coefficients. Its computational complexity per symbol is shown in Table 3-3.

\subsubsection{Complexity of IC}

For the IC in the time domain, the $p$-filter has $L+1$ taps and the $q$-filter has $2 L$ taps. One extra addition per symbol is required to subtract the $q$-filter output from the p-filter output. The complexity is shown in Table 3-4 and 3-5. In the frequency domain, the $P$-filter and the $Q$-filter each require one FFT and one complex multiplication to apply the filter coefficients. A complex addition is required to calculate the difference between the two filters, and an IFFT is needed to convert back to the time domain. The complexity is shown in Table 3-6 and 3-7.

To get an idea of the total amount of work that must be done by the receiver for the purposes of equalization for a given number of iterations, note that a LE (either time or frequency domain) is always used for the first iteration, and an IC (either time or frequency domain) is always used on the second and subsequent iterations. Since the output of the $p$-filter ( $P$-filter) does not change with each iteration, its output need only be calculated once, in the second iteration, and stored for reuse in the third and subsequent iterations. For $N_{i} \geq 2$, where $N_{i}$ is the number of iteration, the total equalization complexity is as shown in Table 3-8 and 3-9 for the time and frequency domain, respectively. 


\begin{tabular}{|c|c|c|c|}
\hline & & $R M$ & $R A$ \\
\hline \multirow{2}{*}{$p$-filter } & $(L+1) \times C M$ & $4(L+1)$ & $2(L+1)$ \\
\cline { 2 - 4 } & $L \times C A$ & & $2 L$ \\
\hline total & & $4 L+4$ & $4 L+2$ \\
\hline
\end{tabular}

Table 3-4. Complexity of the $p$-filter in the TD-IC.

\begin{tabular}{|c|c|c|c|}
\hline \multirow{2}{*}{$q$-filter } & $(2 L) \times C M$ & $4(2 L)$ & $R A$ \\
\cline { 2 - 4 } & $(2 L-1) \times C A$ & & $2(2 L)$ \\
\hline extra $C A$ & & & $2(2 L-1)$ \\
\hline total & & $8 L$ & 2 \\
\hline
\end{tabular}

Table 3-5. Complexity of the $q$-filter in the TD-IC.

\begin{tabular}{|c|c|c|c|}
\hline & & $R M$ & $R A$ \\
\hline \multirow{3}{*}{$P$-filter } & $1 \times \mathrm{FFT}$ & $2 \log _{2} N$ & $3 \log _{2} N$ \\
\cline { 2 - 4 } & $1 \times C M$ & 4 & 2 \\
\hline total & & $2 \log _{2} N+4$ & $3 \log _{2} N+2$ \\
\hline
\end{tabular}

Table 3-6. Complexity of the $P$-filter in the FD-IC. 


\begin{tabular}{|c|c|c|c|}
\hline \multirow{2}{*}{$q$-filter } & & $R M$ & $R A$ \\
\cline { 2 - 4 } & $1 \times \mathrm{FFT}$ & $2 \log _{2} N$ & $3 \log _{2} N$ \\
\hline extra $C A$ & $1 \times C M$ & 4 & 2 \\
\hline IFFT & $1 \times \mathrm{FFT}$ & $2 \log _{2} N$ & 2 \\
\hline total & & $4 \log _{2} N+4$ & $6 \log _{2} N$ \\
\hline
\end{tabular}

Table 3-7. Complexity of the $Q$-filter in the FD-IC.

\begin{tabular}{|c|c|c|}
\hline & $R M$ & $R A$ \\
\hline TD-LE & $32 L+4$ & $32 L+2$ \\
\hline$p$-filter & $4 L+4$ & $4 L+2$ \\
\hline$q$-filter & $8 L\left(N_{i}-1\right)$ & $8 L\left(N_{i}-1\right)$ \\
\hline total & $8 L N_{i}+28 L+8$ & $8 L N_{i}+28 L+4$ \\
\hline
\end{tabular}

Table 3-8. Complexity of the TD-IC.

\begin{tabular}{|c|c|c|}
\hline & $R M$ & $R A$ \\
\hline FD-LE & $4 \log _{2} N+4$ & $6 \log _{2} N+2$ \\
\hline$P$-filter & $2 \log _{2} N+4$ & $3 \log _{2} N+2$ \\
\hline$Q$-filter & $\left(4 \log _{2} N+4\right)\left(N_{i}-1\right)$ & $\left(6 \log _{2} N+4\right)\left(N_{i}-1\right)$ \\
\hline total & $\left(4 \log _{2} N+4\right) N_{i}+2 \log _{2} N+4$ & $\left(6 \log _{2} N+4\right) N_{i}+3 \log _{2} N$ \\
\hline
\end{tabular}

Table 3-9. Complexity of the FD-IC. 


\subsubsection{Complexity of the hybrid IC}

The complexity of the hybrid IC is dependent on the number of pairs of taps that are used in the $q$-filter, $N_{t}$. The complexity of the $P$-filter is shown in Table $3-10$, and the complexity of the $q$-filter is shown in Table $3-11$. The total equalization complexity for $N_{i} \geq 2$ iteration is shown in Table 3-12.

\subsubsection{Comparison}

The total equalization complexity for the three schemes (TD-IC, FD-IC and hybrid IC) is summarized in Table 3-13. Let us consider a numerical example. When $L$ $=9$, and $N_{i}=5$, the complexity of TD-IC is $620 R M$ and $616 R A$. From this example, we see that the complexity is pretty high for both multiplications and additions. If $L$ becomes larger, the complexity will be even worse. Compare this with the complexity of FD-IC. When $N=1024, N_{i}=5$, the complexity of FD-IC is 244 $R M$ and $350 R A$. We can see the complexity of FD-IC is lower than TD-IC. Similarly, let us compute the complexity of the hybrid IC. When $N=1024, N_{i}=5$ and $N_{t}=2$, the complexity of the hybrid IC is $152 R M$ and $188 R A$. We can see the complexity of the hybrid IC is lower than FD-IC, and much lower than TD-IC.

The computational complexity of the FD-IC and the hybrid IC are shown in Figure 3-5 as a function of the block size $(N)$. It is assumed that the hybrid IC uses one pair of coefficients $\left(N_{t}=1\right)$ and five iterations are done $\left(N_{i}=5\right)$. Also shown is the complexity of TD-IC (with $L=4$ ), which does not depend on the block size. Increasing $L$ will increase the complexity of TD-IC, and increasing $N_{t}$ will increase the complexity of the hybrid IC which eventually will be more complex than FD-IC. 


\begin{tabular}{|c|c|c|c|}
\hline & & $R M$ & $R A$ \\
\hline \multirow{3}{*}{$p$-filter } & $2 \times \mathrm{FFT}$ & $4 \log _{2} N$ & $6 \log _{2} N$ \\
\cline { 2 - 4 } & $1 \times C M$ & 4 & 2 \\
\hline total & & $4 \log _{2} N+4$ & $6 \log _{2} N+2$ \\
\hline
\end{tabular}

Table 3-10. Complexity of the $P$-filter in the hybrid IC.

\begin{tabular}{|c|c|c|c|}
\hline & & $R M$ & $R A$ \\
\hline \multirow{2}{*}{$q$-filter } & $2 N_{t} \times C M$ & $4 \times 2 N_{t}$ & $2 \times 2 N_{t}$ \\
\hline & $\left(2 N_{t}-1\right) \times C A$ & & $2 \times\left(2 N_{t}-1\right)$ \\
\hline extra $C A$ & & & 2 \\
\hline total & & $8 N_{t}$ & $8 N_{t}$ \\
\hline
\end{tabular}

Table 3-11. Complexity of the $q$-filter in the hybrid IC.

\begin{tabular}{|c|c|c|}
\hline & $R M$ & $R A$ \\
\hline FD-LE & $4 \log _{2} N+4$ & $6 \log _{2} N+2$ \\
\hline$P$-filter & $4 \log _{2} N+4$ & $6 \log _{2} N+2$ \\
\hline$q$-filter & $8 N_{t}\left(N_{i}-1\right)$ & $8 N_{t}\left(N_{i}-1\right)$ \\
\hline total & $8 \log _{2} N+8+8 N_{t}\left(N_{i}-1\right)$ & $12 \log _{2} N+4+8 N_{t}\left(N_{i}-1\right)$ \\
\hline
\end{tabular}

Table 3-12. Complexity of the hybrid IC. 


\begin{tabular}{|c|c|c|}
\hline & $R M$ & $R A$ \\
\hline TD-IC & $8 L N_{i}+28 L+8$ & $8 L N_{i}+28 L+4$ \\
\hline FD-IC & $\left(4 \log _{2} N+4\right) N_{i}+2 \log _{2} N+4$ & $\left(6 \log _{2} N+4\right) N_{i}+3 \log _{2} N$ \\
\hline hybrid-IC & $8 \log _{2} N+8+8 N_{t}\left(N_{i}-1\right)$ & $12 \log _{2} N+4+8 N_{t}\left(N_{i}-1\right)$ \\
\hline
\end{tabular}

Table 3-13. Complexity comparison.

Increasing $N_{t}$ will magnify the differences between the systems.

Note that in all the analysis of this section we have ignored the complexity involved in calculating the filter coefficients. In particular, calculating the coefficients for the hybrid IC can require significant effort, since an IFFT and two matrix inversions are required (although the inversion are for small $N_{t} \times N_{t}$ matrices). However, depending on the rate at which the channel coefficients are changing (the fading rate), it may only be necessary to calculate the coefficients once for every several transmitted blocks. Therefore, the additional complexity has been omitted. 

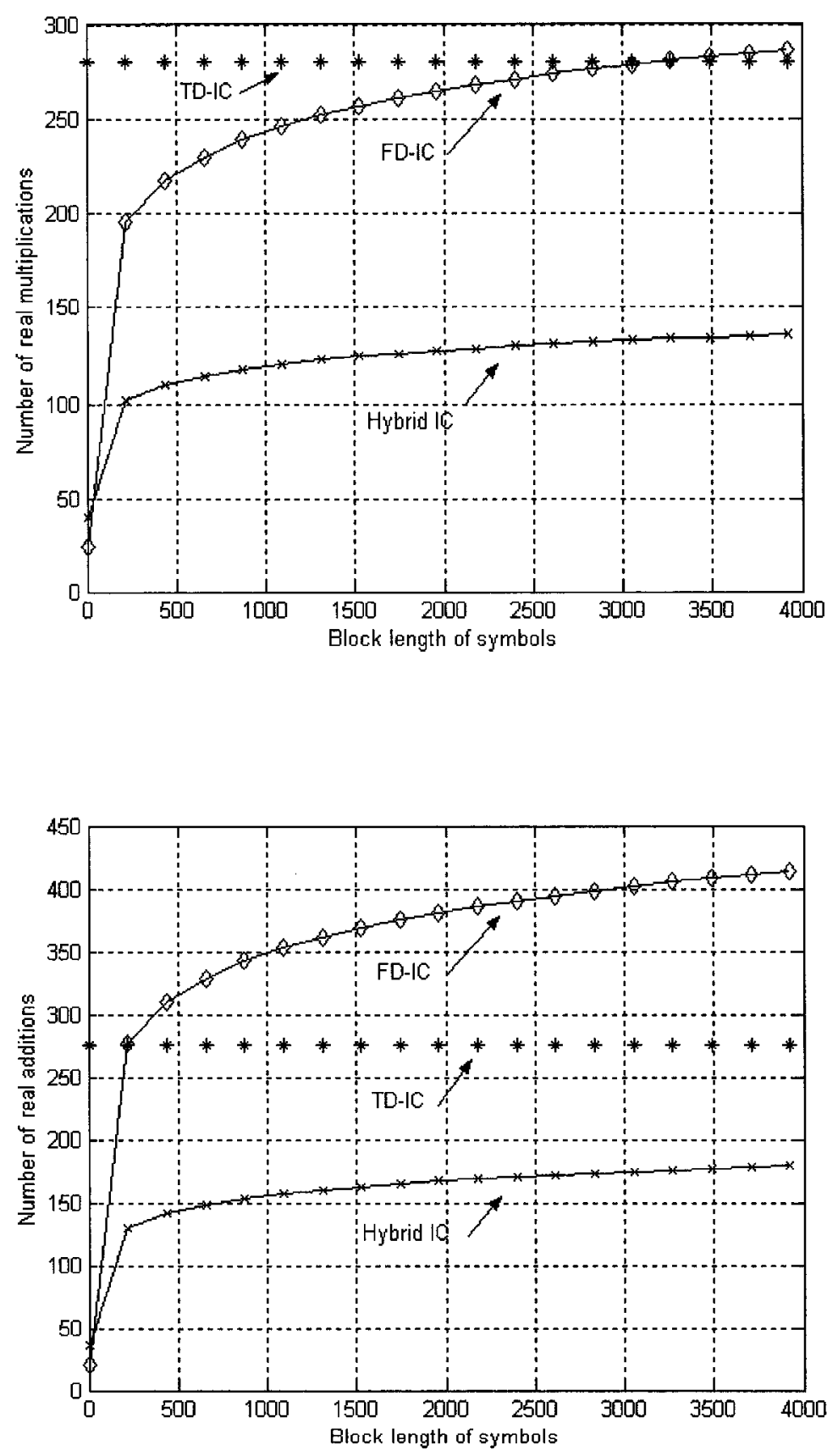

Figure 3-5. Computational complexity of TD-IC, FD-IC and hybrid IC. 


\section{Chapter 4 \\ SIMULATION RESULTS}

In this chapter, simulation results for IC-based turbo equalization in the frequency domain, called FD-IC, are presented. One purpose of these simulations is to verify that operating in the frequency domain is as effective at eliminating ISI as in the time domain. The other purpose is to test the performance of the proposed hybrid reduced complexity system derived in Chapter Three.

For all simulations, message blocks of length $N_{a}=1020$ binary symbols are encoded using a rate $1 / 2$ convolutional code with generator $(23,35)$ (expressed in octal), giving $N_{c}=1024$. The number of simulation block is more than 10000 at BER of $10^{-5}$ and may be decreased at BER more than $10^{-5}$. The symbol interleaver uses a randomly selected permutation and the interleaved code symbols are transmitted over the discrete-time ISI channel. Root raised cosine pulse shaping is used by the transmit filter with a roll-off factor of $\alpha=0.1$.

In this chapter, Section 4.1 investigates the performances for FD-IC over static ISI channels. Section 4.2 discusses the performance of FD-IC over Rayleigh frequency selective fading channels. Section 4.3 inspects the proposed hybrid reduced complexity IC over static ISI and Rayleigh frequency-selective fading channels.

\subsection{Performance of FD-IC over Static ISI Channels}

For the FD-IC turbo equalizer, the LE is employed at the first iteration. For the following iterations, the FD-IC is used. The FD-IC model is shown in Figure 3-3. The 
performance curves of BER and FER versus $E_{b} / N_{0}$ are plotted for the system model. To show the performance degradation due to ISI, the BER and FER of an ISI-free coded data transmission system is also plotted. Although the advantage of frequency domain equalization, in terms of complexity, is evident, primarily in channels with long delay spreads, it is useful to begin with a short delay spread channel.

\subsubsection{Proakis B channel}

The impulse and frequency responses of the Proakis B channel [8] are shown in Figure 4-1. As shown in Figure 4-2, the BER performance of the FD-IC improves with each iteration of the equalizer. The system suffers a $8.5 \mathrm{~dB}$ ISI penalty at a BER of $10^{-4}$ after the first iteration compared with the performance with no-ISI. After the second iteration, the ISI penalty is reduced to about $2.5 \mathrm{~dB}$. Further iterative processing gives an overall improvement of $8 \mathrm{~dB}$ at a BER of $10^{-4}$, and almost completely eliminates the ISI. In Figure 4-2 the BER performance of the TD-IC is also plotted [31], and we can see that there is little difference between the performance of TD-IC and FD-IC.

As shown in Figure 4-3, the FER performance also improves with each iteration of the equalizer. After five iterations, the ISI penalty is reduced by $8.5 \mathrm{~dB}$ at a FER of $10^{-2}$ compared to the first iteration.

\subsubsection{Proakis $C$ channel}

The impulse and frequency responses of the Proakis C channel [8] are shown in Figure 4-4. As shown in Figure 4-5, the BER performance improves with each iteration of the equalizer. The system suffers a $17 \mathrm{~dB}$ ISI penalty at a BER of $10^{-4}$ after the first 


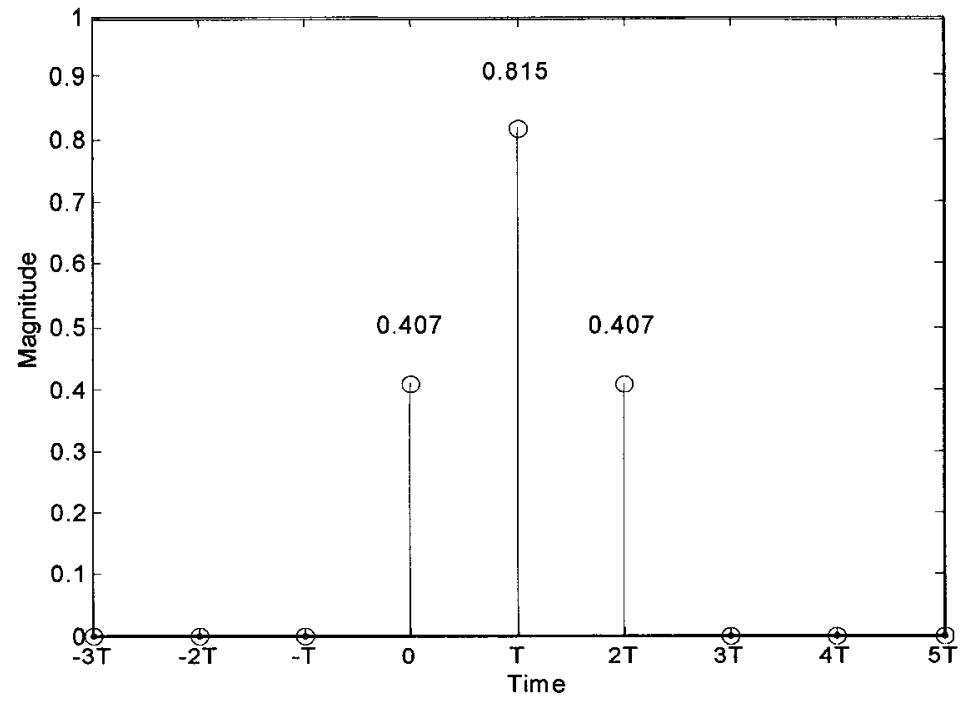

(a) Impulse response.

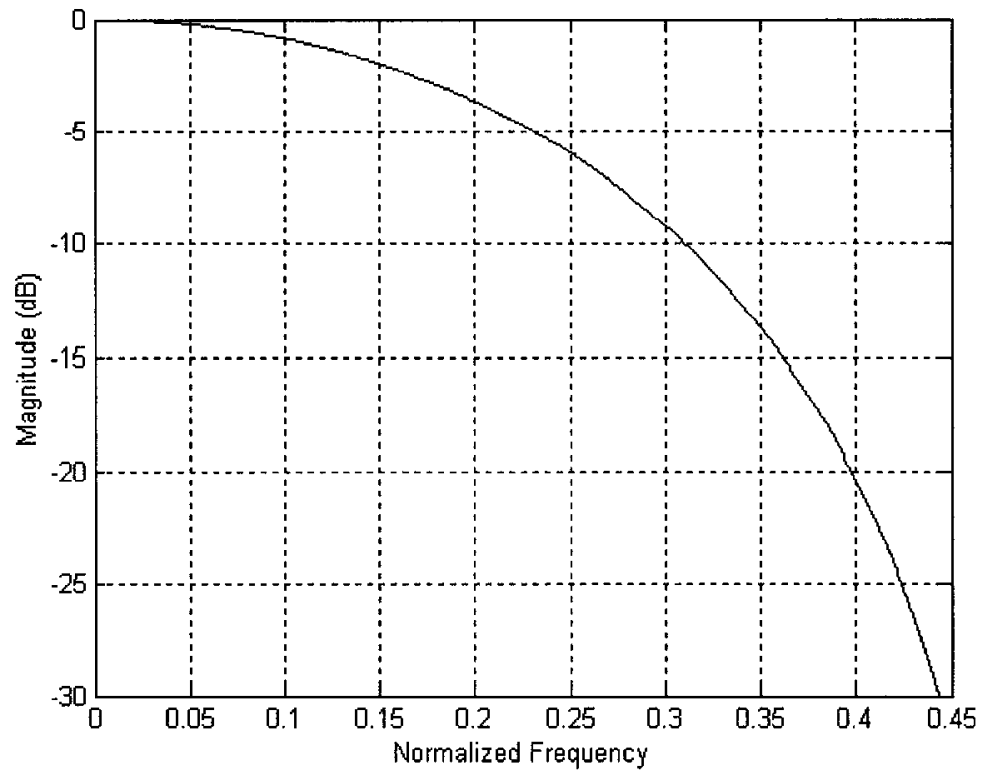

(b) Frequency response.

Figure 4-1. Impulse response (a) and frequency response (b) of the Proakis B channel. $T$ is the symbol duration. 


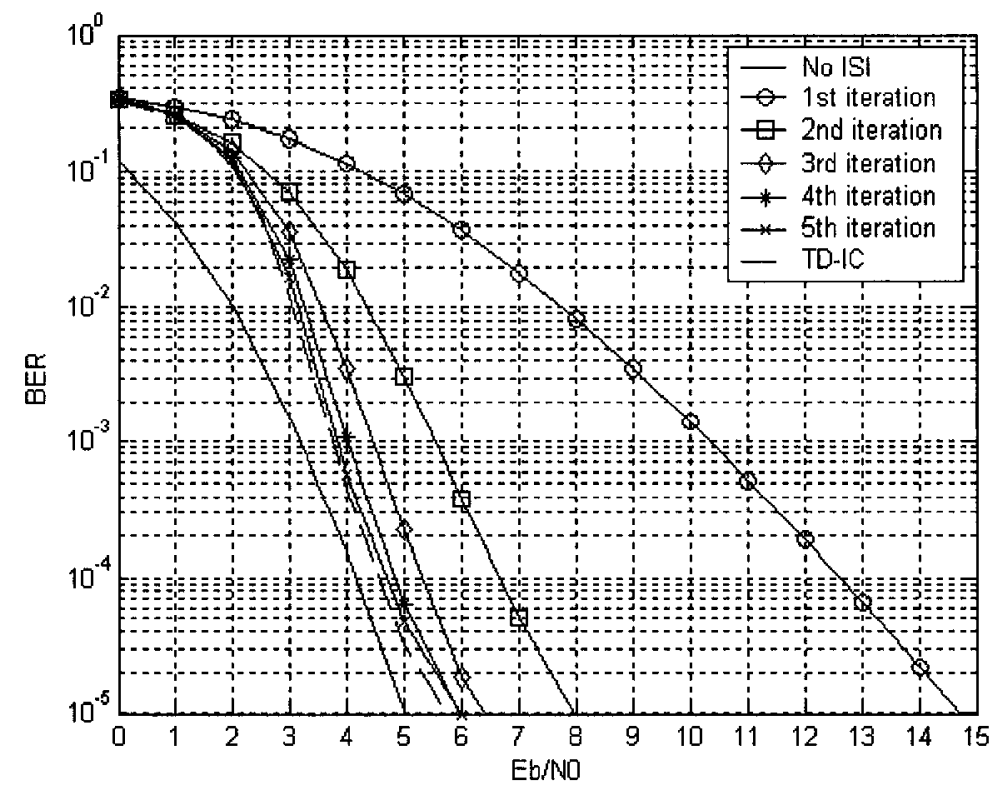

Figure 4-2. BER performance of the FD-IC over the Proakis B channel.

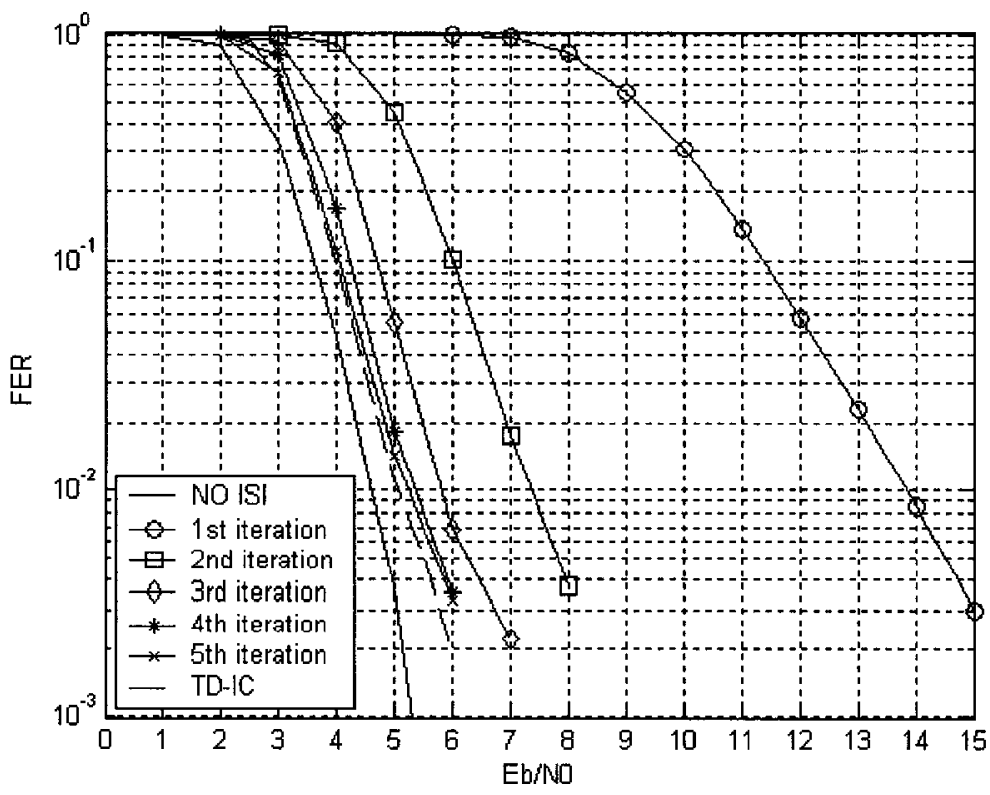

Figure 4-3. FER performance of the FD-IC over the Proakis B channel. 
iteration, which is reduced to $8.5 \mathrm{~dB}$ after five iterations. Unlike for the Proakis $\mathrm{B}$ channel, turbo equalization is not able to completely eliminate the ISI for the Proakis C channel since the Proakis $\mathrm{C}$ channel is much more severe than the Proakis B channel. In Figure 4-5, the BER performance of the TD-IC over Proakis $\mathrm{C}$ channel is also plotted and again we can see that there is little difference between the performance of TD-IC and FD-IC, except of high SNR where TD-IC appears to suffer from an error floor. As shown in Figure 4-6, performance on terms of the FER is similar to that of the BER.

\subsubsection{Two-tap channel with a long delay spread}

As shown in Section 3.4, time domain equalization requires many multiplication operations for channels with a long delay spread. For example, for the channel in Figure 4-7, as least several hundred multiplications are needed per symbol. The high computational complexity limits the application of time domain equalization, so it is wiser to use frequency domain equalization instead.

The impulse and frequency responses of a channel with a delay spread of 100 symbols are shown in the Figure 4-7. The results in Figure 4-8 show that the BER performance of the FD-IC improves with each iteration. The system suffers from a 3.5 $\mathrm{dB}$ ISI penalty at a BER of $10^{-4}$ after the first iteration, and after the third iteration, the ISI is completely eliminated for $E_{b} / N_{0}$ greater than $3 \mathrm{~dB}$. Similar results hold with regard to the FER, as shown in Figure 4-9. Frequency domain turbo equalization is clearly able to handle channels with long delay spreads and with low complexity. 


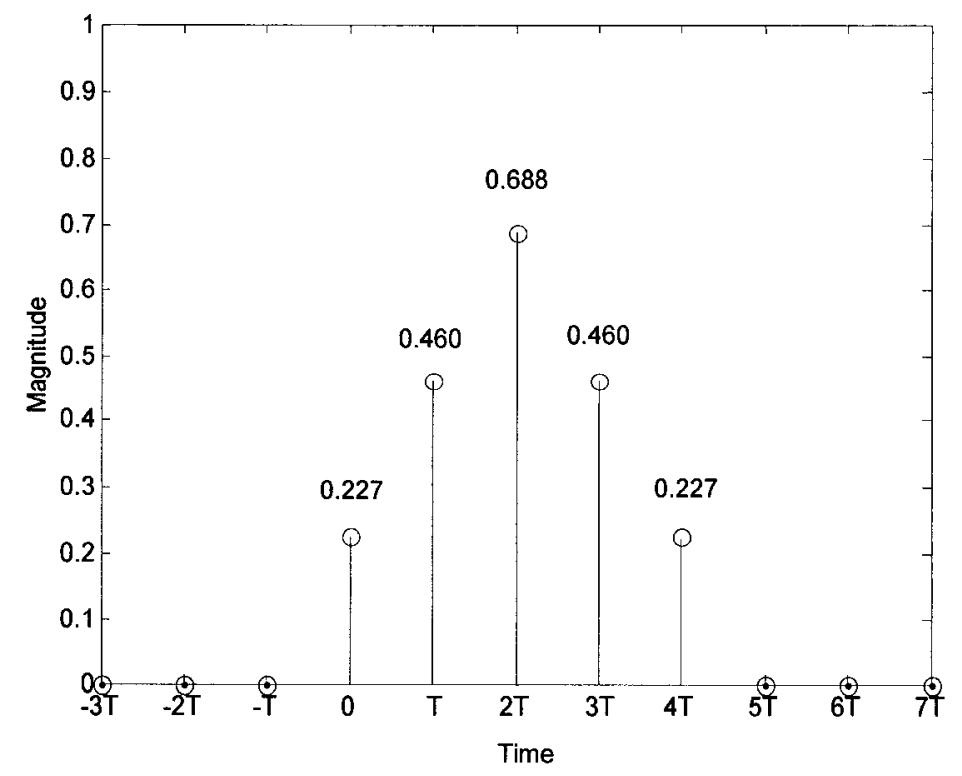

(a) Impulse response.

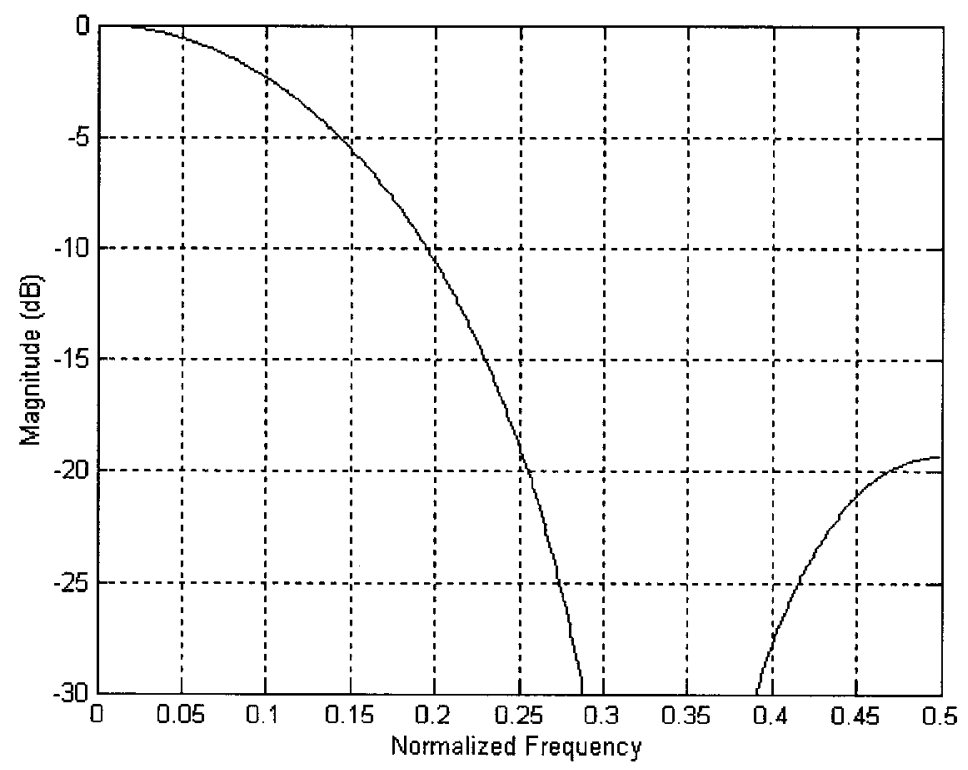

(b) Frequency response.

Figure 4-4. Impulse response (a) and frequency response (b) of the Proakis C channel. 


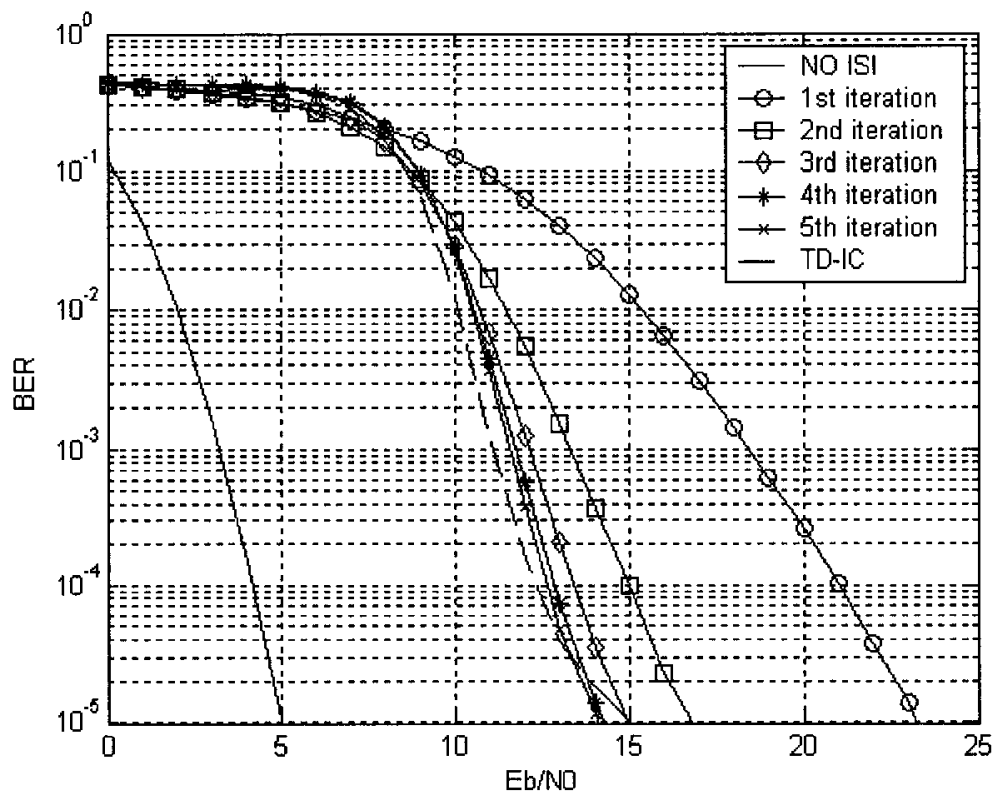

Figure 4-5. BER performance of the FD-IC over the Proakis C channel.

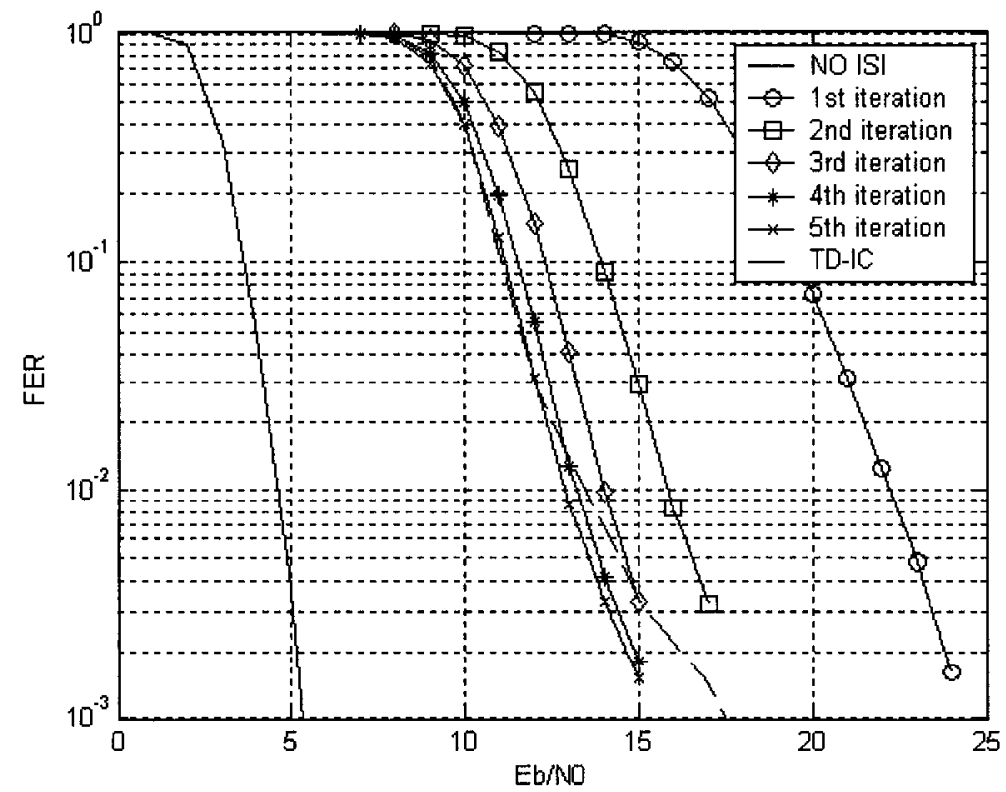

Figure 4-6. FER performance of the FD-IC over the Proakis C channel. 


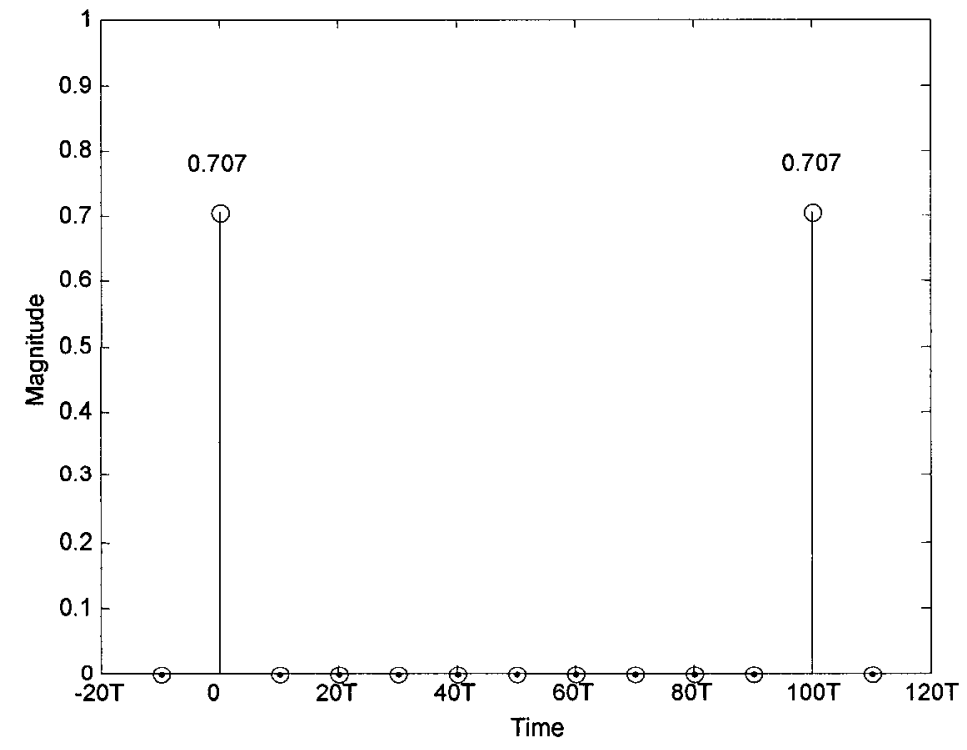

(a) Impulse response.

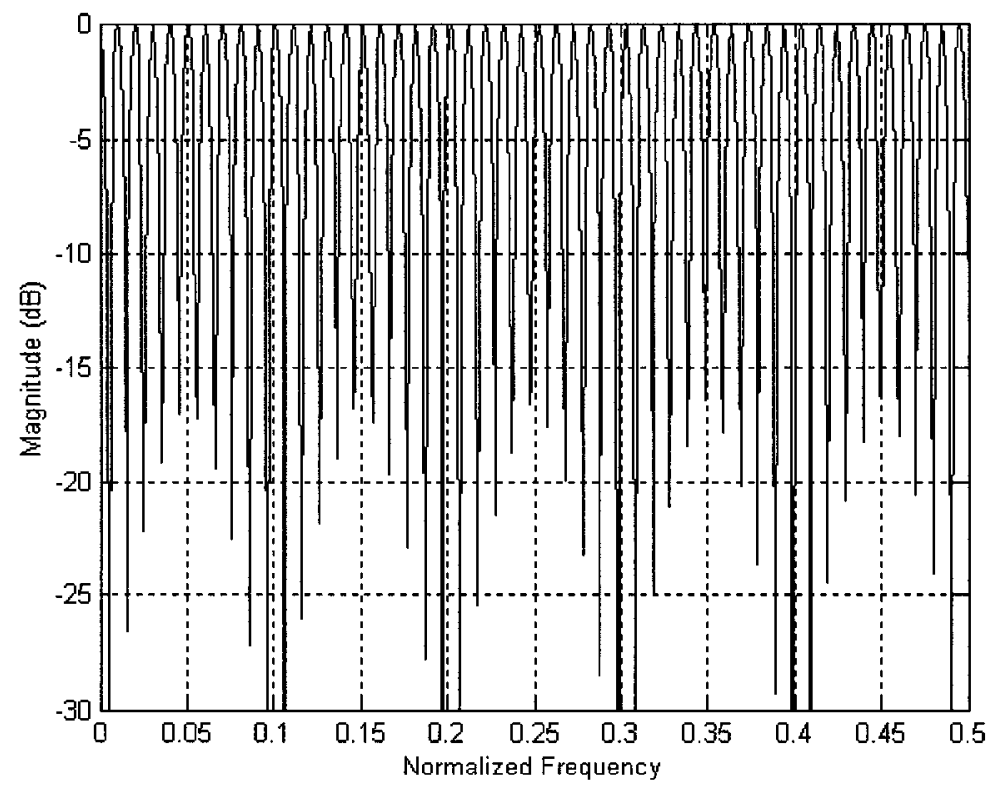

(b) Frequency response.

Figure 4-7 Impulse (a) and frequency (b) response of a 2-tap channel with long delay spread. 


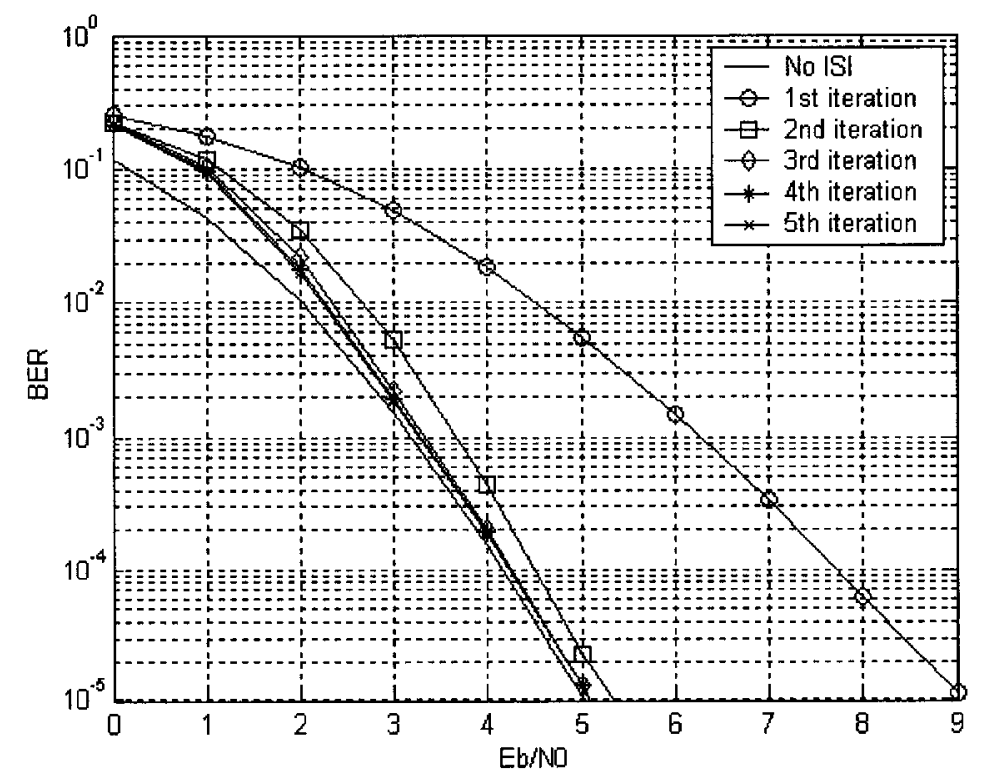

Figure 4-8. BER performance of the FD-IC over 2-tap channel with long delay spread.

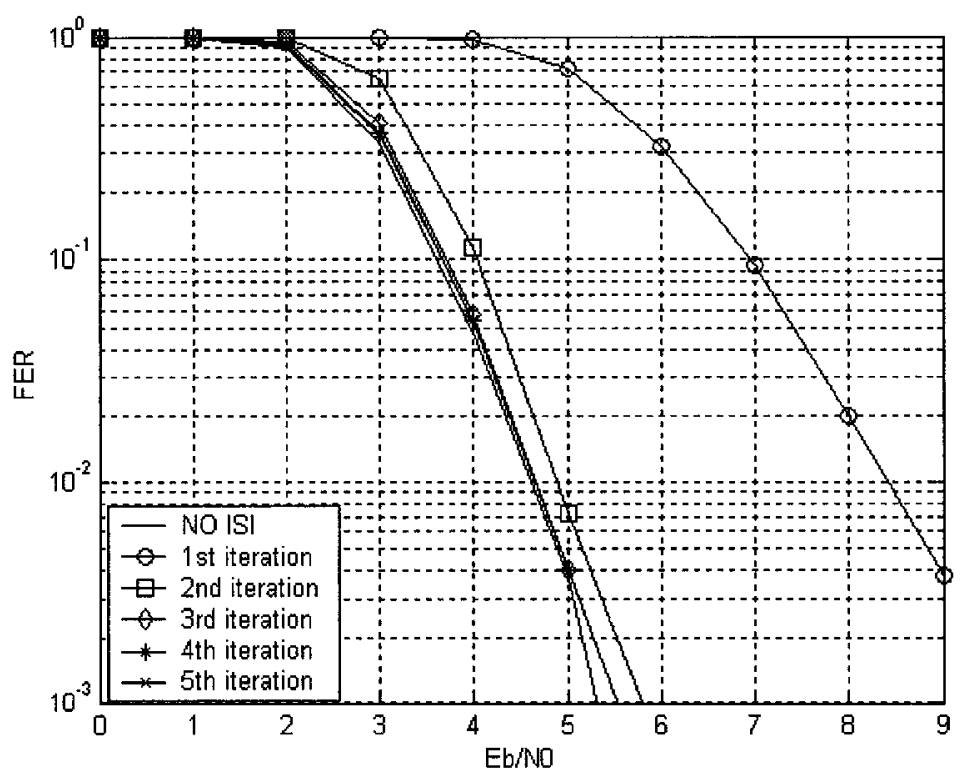

Figure 4-9. FER performance of the FD-IC over 2-tap channel with long delay spread. 


\subsection{Performance over Rayleigh Frequency Selective Fading Channels}

In this section, we investigate the BER and FER performances of the FD-IC over Rayleigh frequency-selective fading channels, such as the SUI-5, SUI-6 and 10-tap channels.

\subsubsection{SUI-5 and SUI-6 channels}

The standardization of fixed broadband wireless access (BWA) systems is carried out by the IEEE 802.16 Working Group [30]. They consider six different radio channel models for BWA in three terrain categories. In this thesis, the SUI-5 channel and SUI- 6 channel models with long delay spreads and with the use of omnidirectional antenna in terrain type A are considered. Their delay spread profiles are shown in Figure 4-10 and Figure 4-11. Both of these channels have three paths $\left(N_{p}=3\right)$ with different attenuation $\left(\beta_{i}\right)$ and delay spread $\left(\tau_{i}\right)$ for each path. $\beta_{i}$ is modeled as a complex Gaussian random variable, with zero mean and a variance of $\sigma_{i}^{2}=E\left[\left|\beta_{i}\right|^{2}\right]$. When a symbol rate of 1 Mbaud is assumed, $\sigma_{i}^{2}$ and $\tau_{i}$ of the SUI-5 and SUI-6 channels are as shown in Table 4-1.

The BER performance of the FD-IC with QPSK for the SUI-5 channel is shown in Figure 4-12. Although there is a $1 \mathrm{~dB}$ iteration gain from using two iterations instead of one, further gains are not possible and a $1 \mathrm{~dB}$ ISI penalty remains after five iterations compared to the matched filter bound (MFB). The MFB represents the performance of an optimal receiver for the same channel and same transmitted signal, except that the symbol rate has been drastically reduced so that ISI does not occur. The FER results in Figure 4-13 show that the FD-IC with two iterations can reach the MFB. Similar results 


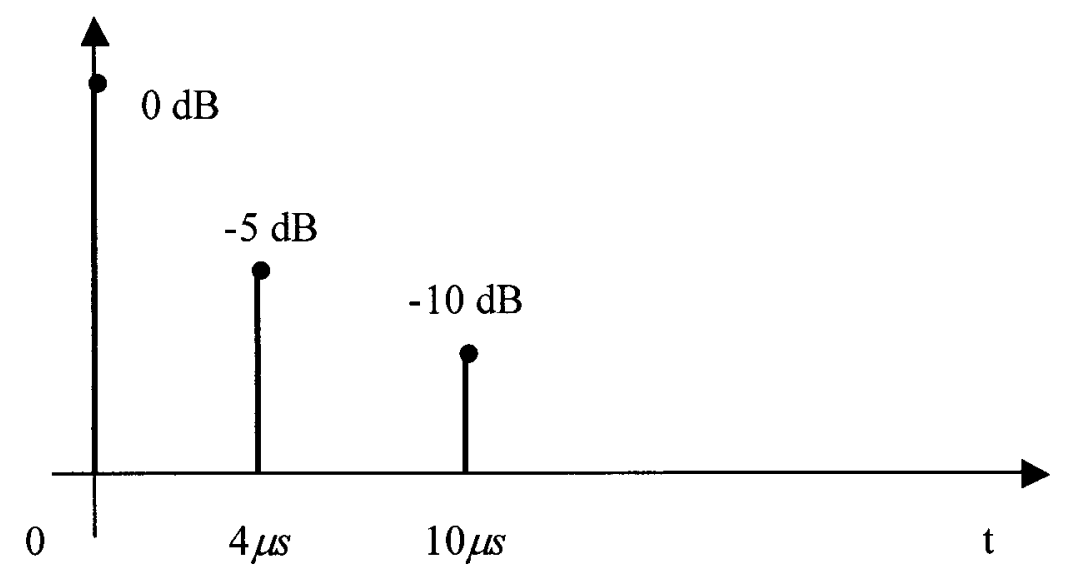

Figure 4-10. Delay spread profile for SUI-5 channel.

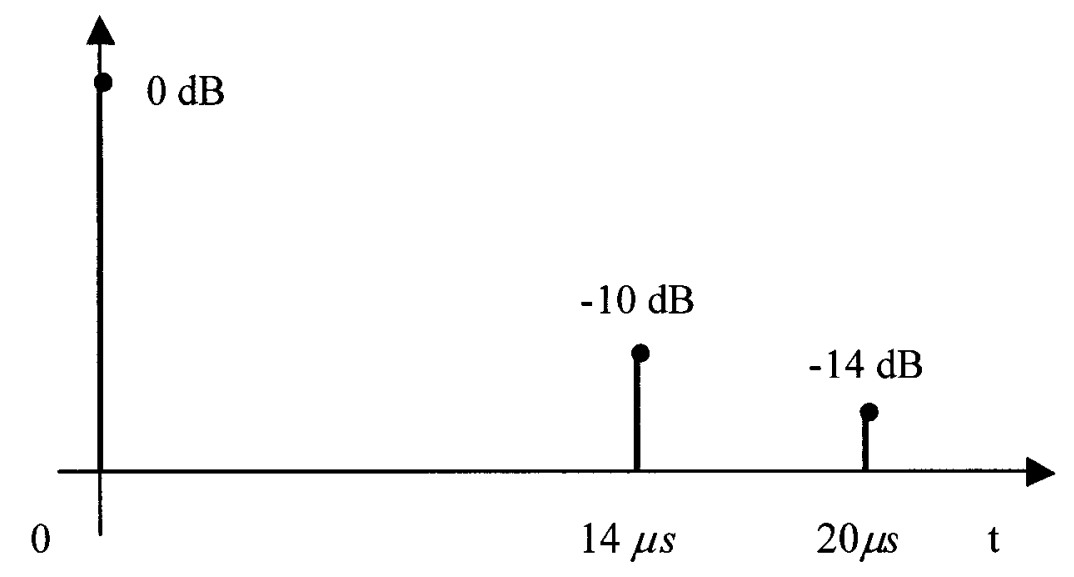

Figure 4-11. Delay spread profile for SUI-6 channel.

\begin{tabular}{|c|c|c|c|c|}
\hline \multirow{2}{*}{ SUI-5 } & $\sigma_{i}^{2}$ & 0.706 & 0.223 & 0.0706 \\
\cline { 2 - 5 } & $\tau_{i}$ & 0 & 4 & 10 \\
\hline \multirow{2}{*}{ SUI-6 } & $\sigma_{i}^{2}$ & 0.877 & 0.0877 & 0.0349 \\
\cline { 2 - 5 } & $\tau_{i}$ & 0 & 14 & 20 \\
\hline
\end{tabular}

Table 4-1. Parameters for the SUI-5 and SUI- 6 channels. 
occur with the SUI-6 channel, as shown in Figure 4-14 and 4-15. Since the effect of ISI can be eliminated in terms of the FER but not the BER, this suggests that, for frames with errors, the average number of bits in error per frame is larger than for the MFB system, although the number of frames with errors is the same. The likely cause of this is that, although the FD-IC is usually able to completely eliminate the ISI, in some cases, such as when the overall channel attenuation is large, iterative processing introduces additional errors.

For the SUI-5 channel we also investigate the performance of the FD-IC when 16-QAM is used with Ungerboeck's 16-state rate-3/4 trellis-coded modulation (TCM) code [31]. The BER results shown in Figure 4-16 are similar to that of the QPSK system, but the iteration gain is slight larger $(2 \mathrm{~dB})$ as is the ISI penalty after five iterations $(1.5 \mathrm{~dB})$. The FER results shown in Figure $4-17$, on the other hand, are notably different. The ISI penalty reduces slowly with each iteration, and even after five iterations there remains a penalty of $0.5 \mathrm{~dB}$.

Overall, the BER performance of the FD-IC shows that turbo equalization does not give the dramatic improvement for the SUI-5 and SUI-6 channels that was evident in the static ISI channels. Nonetheless, the ISI penalty after turbo equalization is quite small, particularly in terms of the FER. Furthermore, it is evident that only two iterations are needed to realize all the benefits of turbo equalization. The only exception is for the FER of the 16-QAM system which does show improvement with each iteration. 


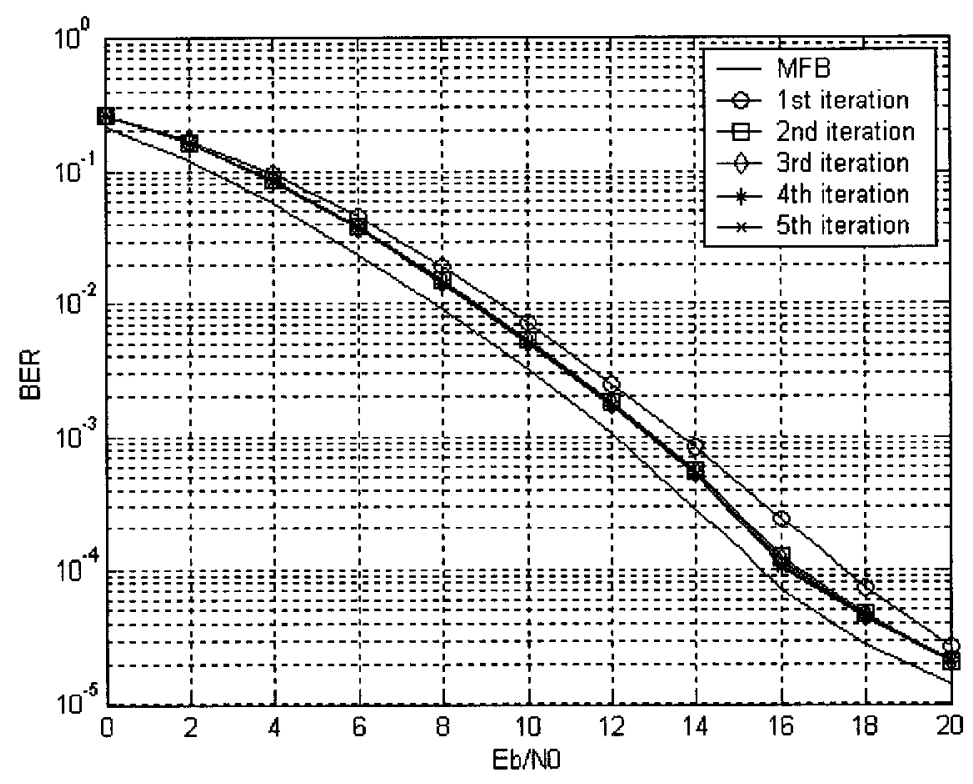

Figure 4-12. BER of the FD-IC for QPSK over the SUI-5 channel.

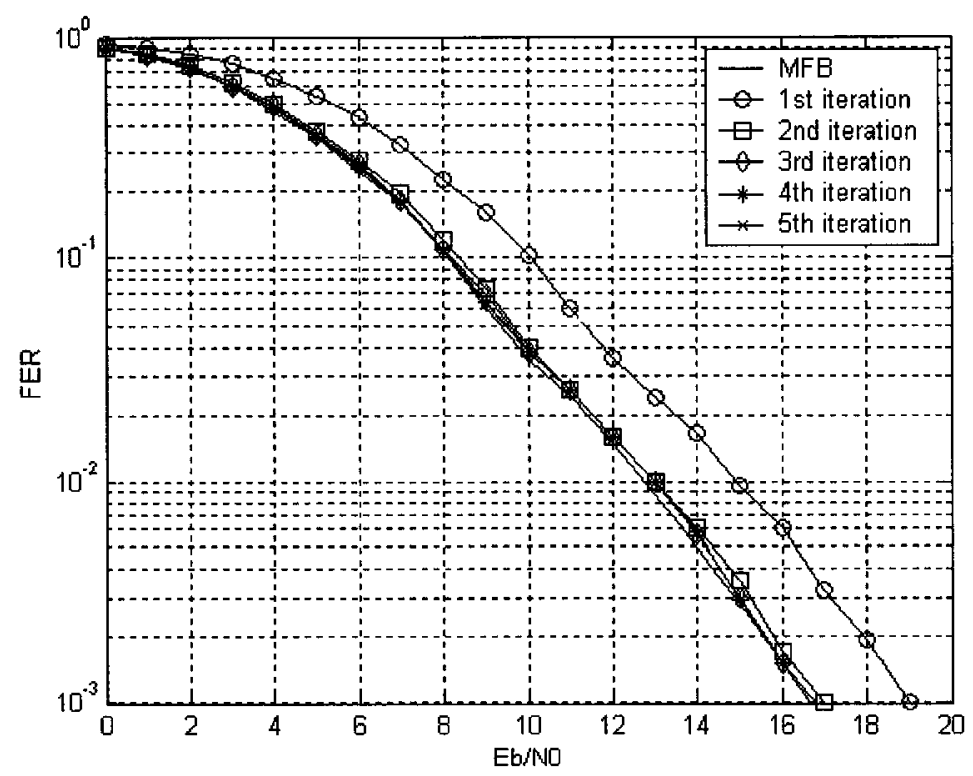

Figure 4-13. FER of the FD-IC for QPSK over the SUI-5 channel. 


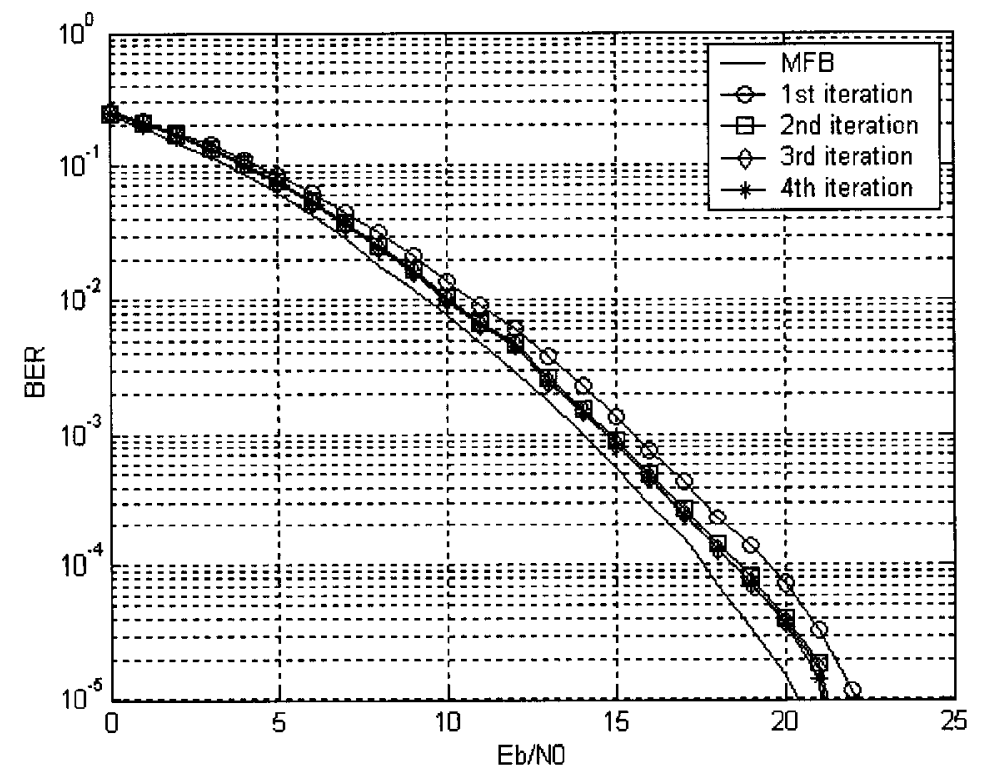

Figure 4-14. BER performance of the FD-IC for QPSK over the SUI-6 channel.

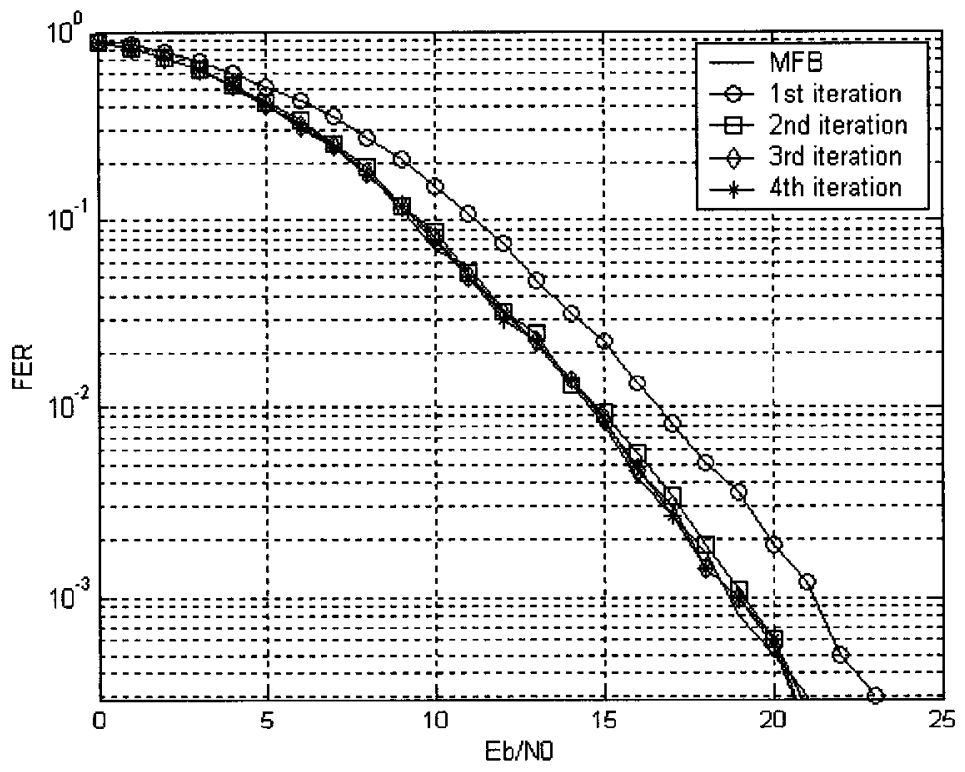

Figure 4-15. FER performance of the FD-IC for QPSK over the SUI-6 channel. 


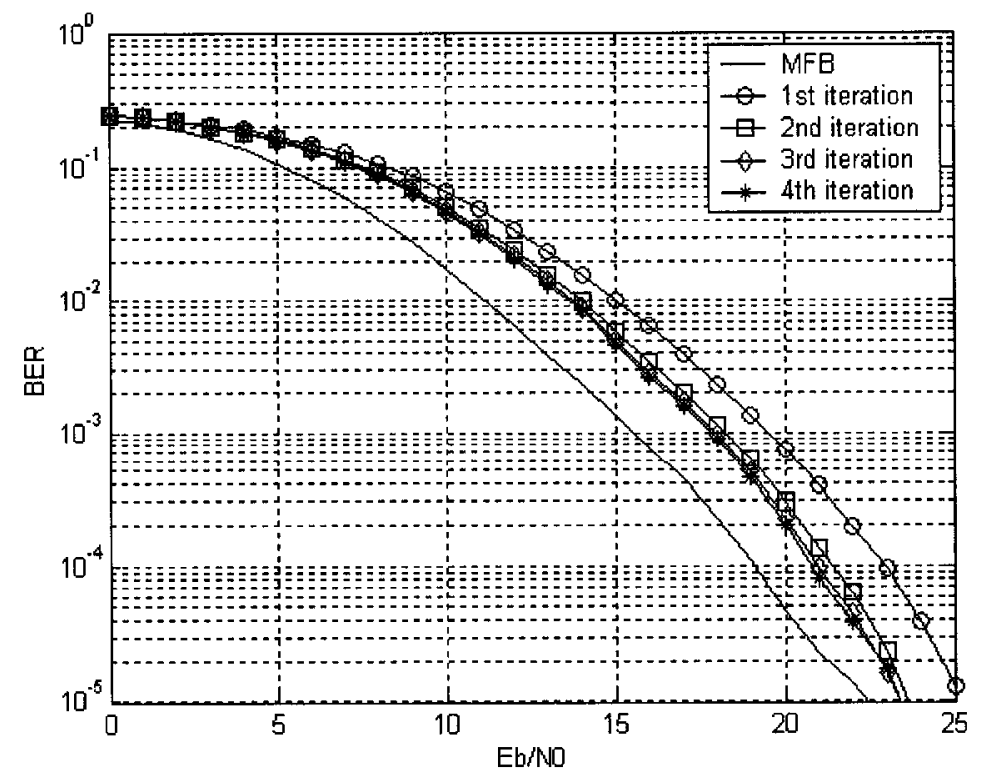

Figure 4-16. BER performance of the FD-IC for 16-QAM over the SUI-5 channel.

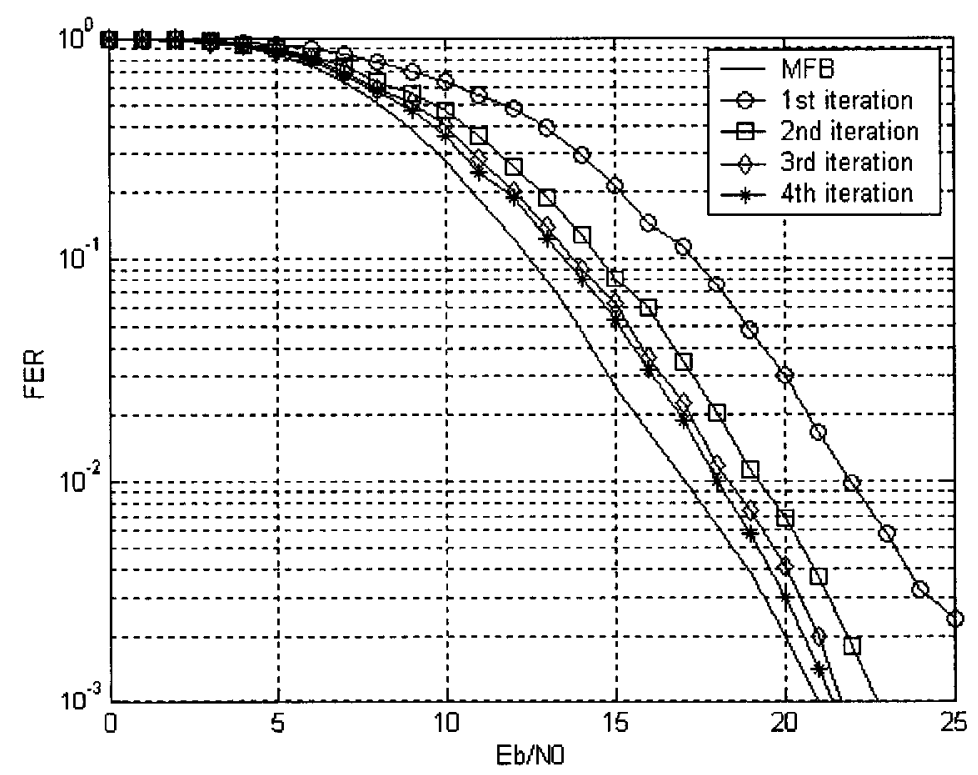

Figure 4-17. FER performance of the original FD-IC for 16-QAM over the SUI-5 channel. 


\subsubsection{0-tap fading channel}

The 10-tap fading channel model consists of ten channel taps, each tap with randomly selected amplitude with a variance of 0.1 . Referring to Section 2.3 .3 , there are delay spread $\tau_{i}=(i-1) T_{s}$ and variance $\sigma_{i}^{2}=0.1$ for $i \in\{1,2, \cdots, 10\}$. We use both QPSK and 16-QAM to investigate the BER and FER performance of the FD-IC.

The BER performance for QPSK can be seen in Figure 4-18. The performance improves with the first three iterations, but then starts to get worse. However, as Figure 4-19 shown, the FER performance continues to improve with each iteration, and after the fourth iteration, the FER performance can almost approach the MFB. This interesting result can be seen more clearly when ten iterations are used, as shown in Figure 4-20 and Figure 4-21. From Figure 4-20, the BER performance becomes worse and worse after the third iteration. Nevertheless, the FER performance still gets better and better as the number of iterations increases. A similar phenomenon can be found for 16-QAM TCM over the same 10-tap channel from the Figure 4-22 and 4-23. Although a small number of message blocks may behave very badly due to the fading channel, causing the whole BER performance to become worse, the overall number of block errors decreases by the iterative process.

In addition, these results show that the FD-IC is much better at combating the ISI when QPSK is used than when 16-QAM is. Although the iterative processing gain is comparable for the two modulation schemes, the residual ISI penalty is much worse for 16-QAM. 


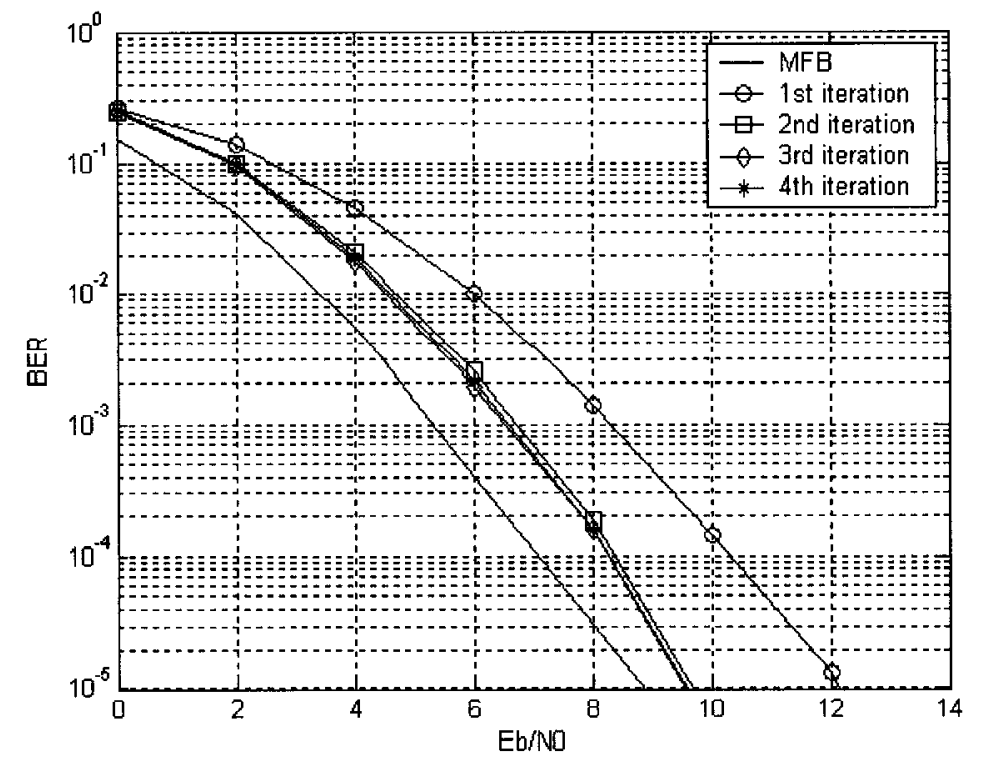

Figure 4-18. BER performance of the FD-IC for QPSK over 10-tap fading channel.

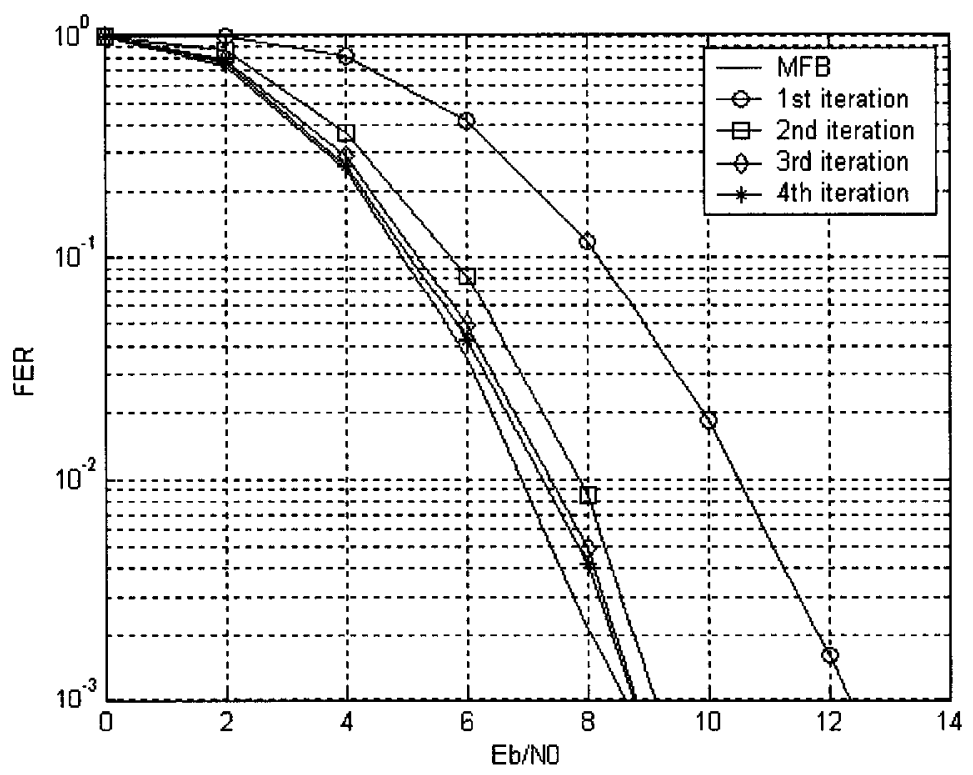

Figure 4-19. FER performance of the FD-IC for QPSK over 10-tap fading channel. 


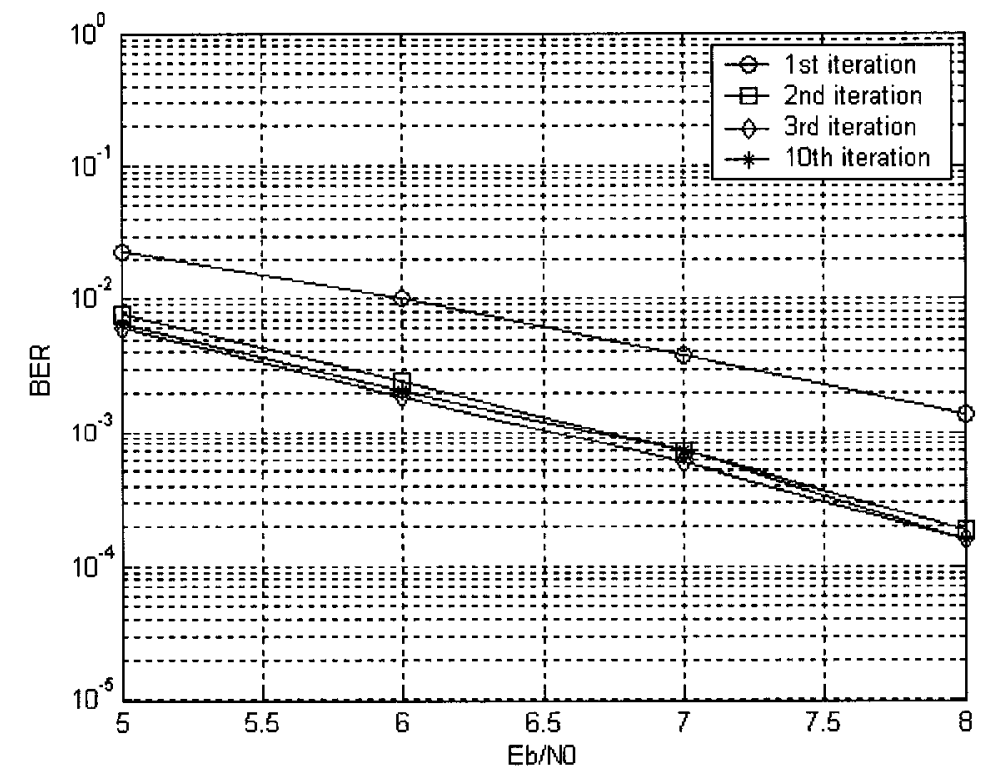

Figure 4-20. BER performance of the FD-IC for QPSK over 10 taps fading channel (with 10th iteration).

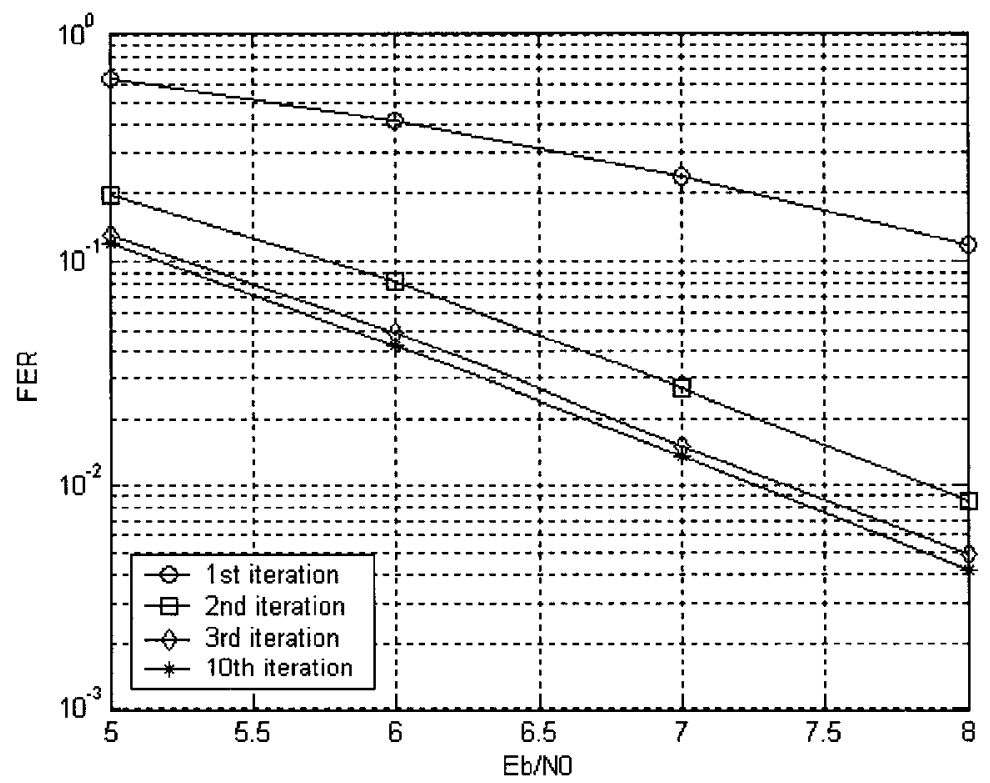

Figure 4-21. FER performance of the FD-IC for QPSK over 10 taps fading channel(with 10th iteration). 


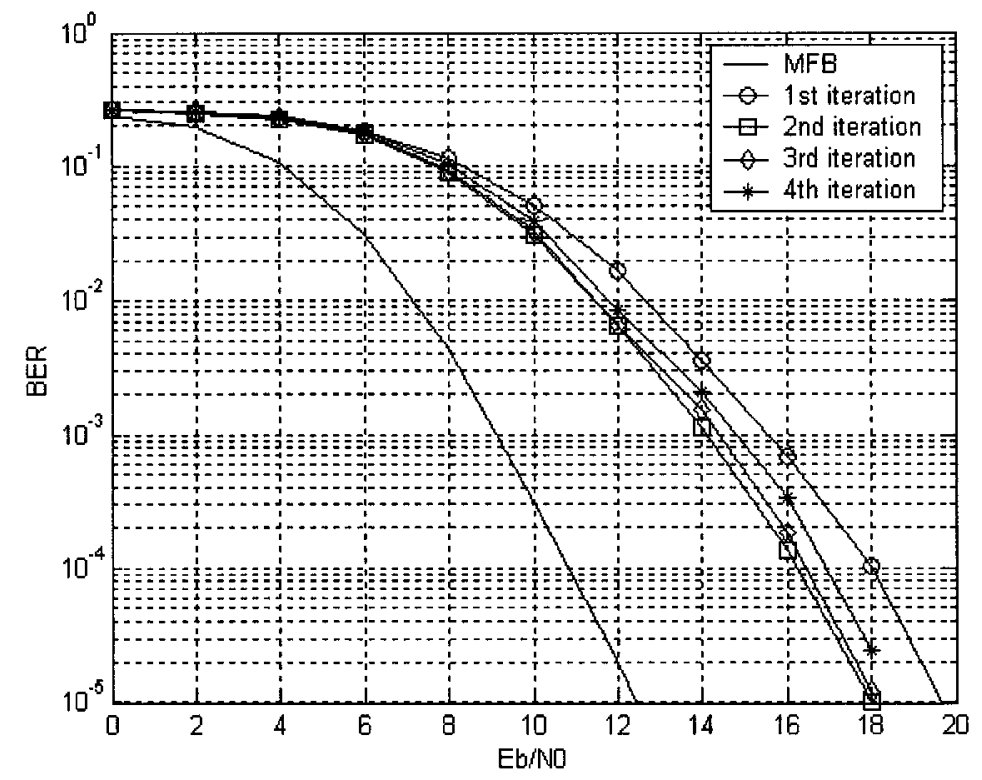

Figure 4-22. BER performance of the FD-IC for 16-QAM over 10-tap fading channel.

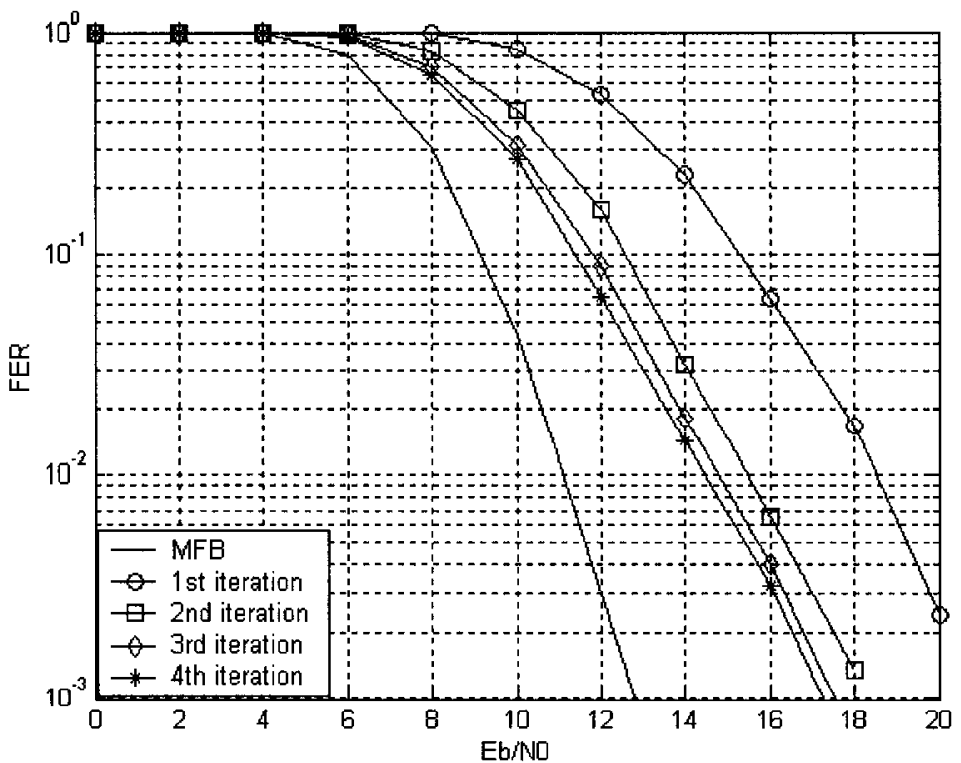

Figure 4-23. FER performance of the FD-IC for 16-QAM over 10-tap fading channel. 


\subsection{Performance of the Hybrid IC}

In terms of complexity, the advantage of using the hybrid IC instead of the FD-IC is mostly apparent when a large number of iterations are required. However, the BER results in the previous section suggest that only two, or perhaps three, iterations are required to realize all the iterative processing gain. Nonetheless, there are cases when more iterations are beneficial, such as when the FER is of interest, or for the static Proakis B and C channels.

In this section, we investigate the BER and FER performances of the hybrid reduced complexity IC over static ISI channel, such as the Proakis B and C channels, and Rayleigh frequency-selective fading channels, such as the SUI-5 and 10-tap channels.

\subsubsection{Performance over static ISI channels}

Figure 4-24 and 4-25 show that BER and FER performance of the hybrid IC system with one pair of taps $\left(N_{t}=1\right)$ for the static Proakis B channel. Also shown is the performance of the original FD-IC after five iterations. As can be seen the hybrid IC system gives almost identical performance as the FD-IC, but with much lower complexity (102 vs. 262 multiplications, and 135 vs. 375 additions). Since $L=2$ for this channel, increasing $N_{t}$ to 2 for the hybrid IC is equivalent to using all the taps, so the performance is then identical to the FD-IC.

The BER and FER results for the Proakis C channel are shown in Figure 4-26 and 4-27 for the hybrid IC with 1, 2, 3, and all four pairs of taps, which is the same as (FDIC). These surprising results show that better results are achieved when only one or two 


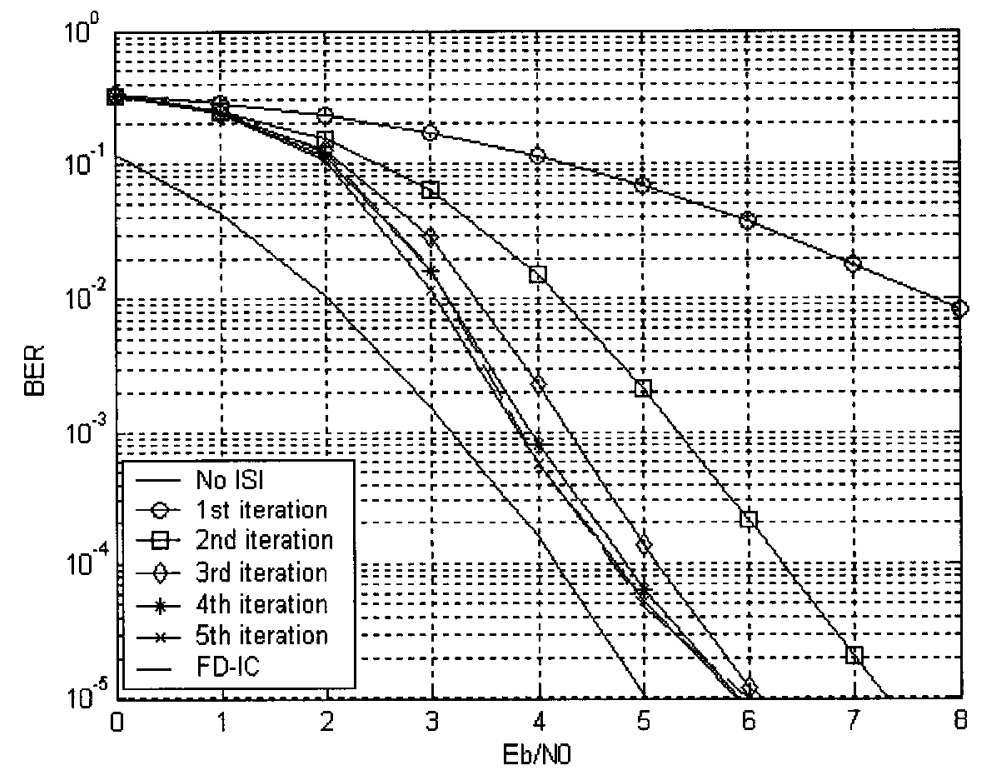

Figure 4-24. BER of the hybrid IC for QPSK over the Proakis B channel.

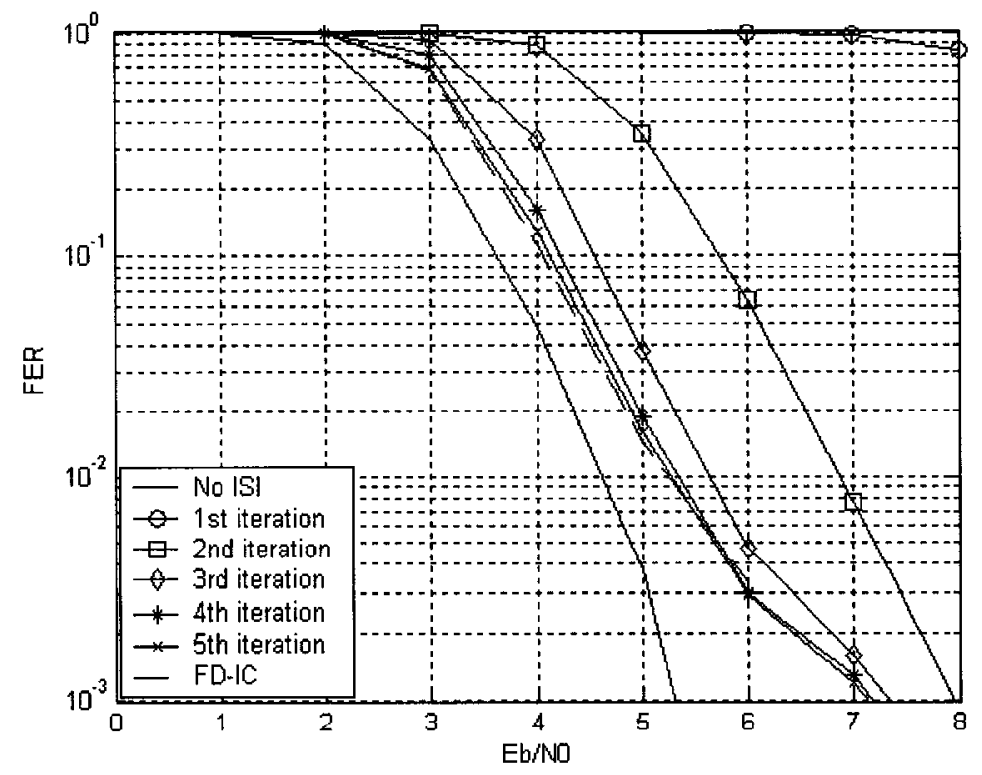

Figure 4-25. FER of the hybrid IC for QPSK over the Proakis B channel. 


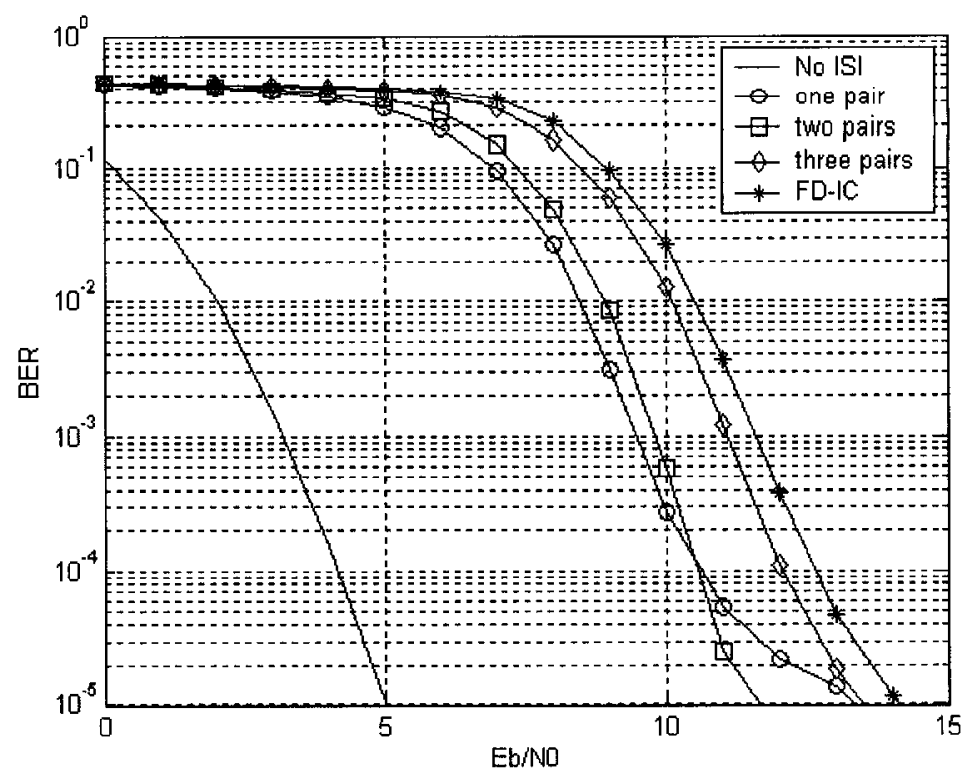

Figure 4-26. BER of the hybrid IC for QPSK over the Proakis C channel.

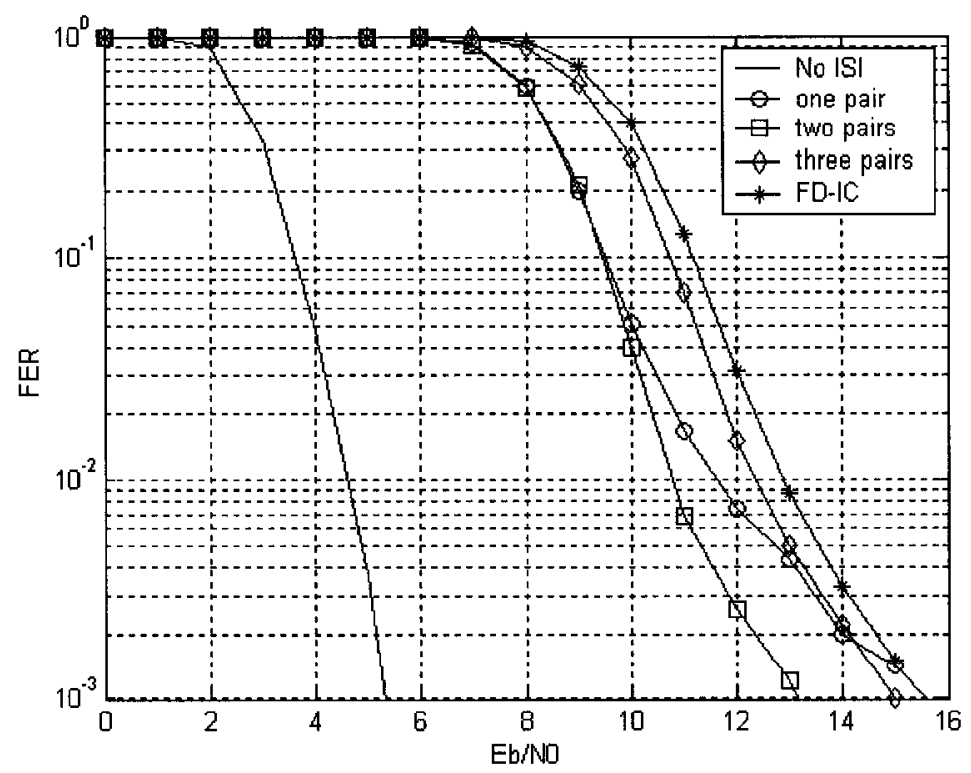

Figure 4-27. FER of the hybrid IC for QPSK over the Proakis C channel. 
taps are used instead of all of them. The hybrid IC is therefore capable of not only providing lower complexity, but also better results. We can explain this case by analysing the structure of FD-IC and the hybrid scheme. The $P$-filter of FD-IC enhances the SNR and the $Q$-filter eliminates the ISI. However, the $P$-filter in the hybrid scheme not only takes on the task as in the FD-IC, but also eliminates the ISI that the $q$-filter cannot remove. On the other hand, if there are errors from previous iteration, the $P$-filter in the hybrid scheme can easily handle a small number of pairs than a large number of pairs. We may also explain this by analyzing Equation (B-2). In Equation (B-2), it is assumed that $v_{n}$ is fed back to the IC, although it might never happen. When the feedback $v_{n}$ contains error, the optimizing $q$-filter for $J=E\left[\left|z_{n}-v_{n}\right|^{2}\right]$ is more prone to error if the filter is longer.

\subsubsection{Performance over the SUI-5 channel}

Figure 4-28 and 4-29 show the BER and FER of the hybrid IC with $N_{t}=1$ for the SUI-5 channel with QPSK. Also shown is the performance of the original FD-IC. In terms of the BER there is very little difference between the hybrid IC and the FD-IC, but in terms of the FER the hybrid IC is about $1 \mathrm{~dB}$ worse. If only the BER is of interest, then it is advantageous to use the hybrid scheme since it has lower complexity, even though only two iterations are required.

\subsubsection{Performance over 10-tap fading channel}

The BER and FER of the hybrid IC after five iterations with one, two, three and all 9 pairs of taps are shown in Figure 4-30 and 4-31 for the 10-tap fading channel with QPSK. Increasing the number of taps leads to better performance, and there is little 
difference between using 3 and 9 pairs in terms of the BER. However, in terms of the FER there is about a $0.8 \mathrm{~dB}$ penalty for using 3 pairs instead of all 9 . There is a clear disadvantage to using fewer pairs.

When 16-QAM is used instead of QPSK, the BER results shown in Figure 4-32 indicates that there is little difference between using 1,2,3 pairs. Furthermore all three of these choices for the number of pairs give better BER performance than the FD-IC. This is not the case when the FER is examined, as shown I Figure 4-33. However, there is little difference between using 2,3 , or 9 pairs. 


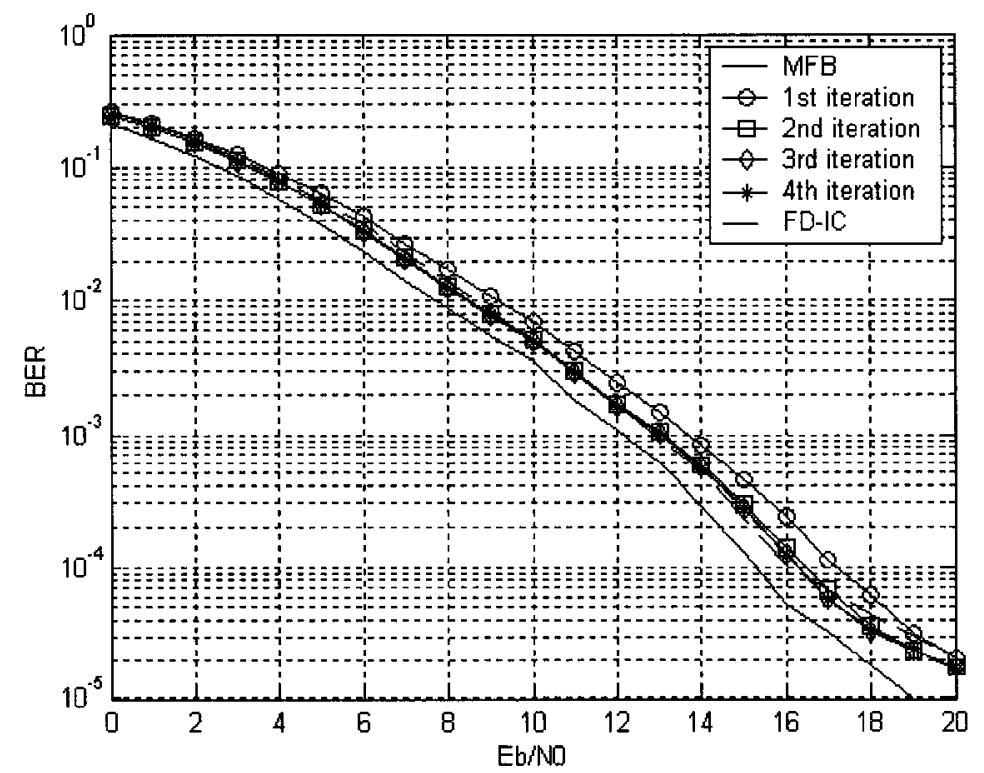

Figure 4-28. BER of the hybrid IC for QPSK over the SUI-5 channel.

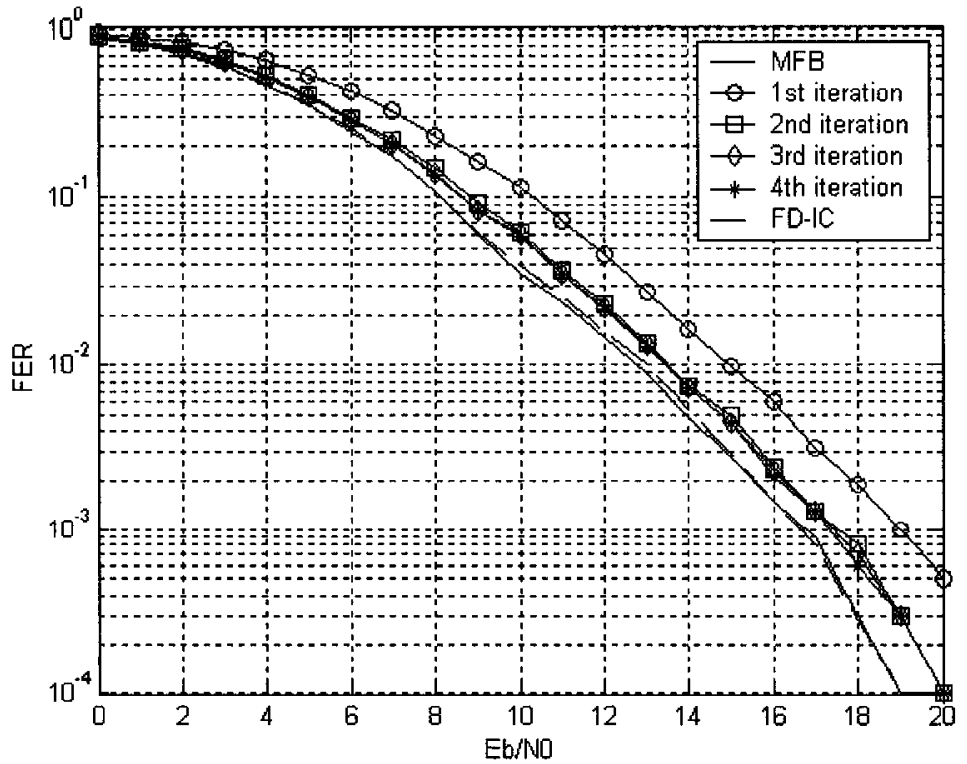

Figure 4-29. FER of the hybrid IC for QPSK over the SUI-5 channel. 


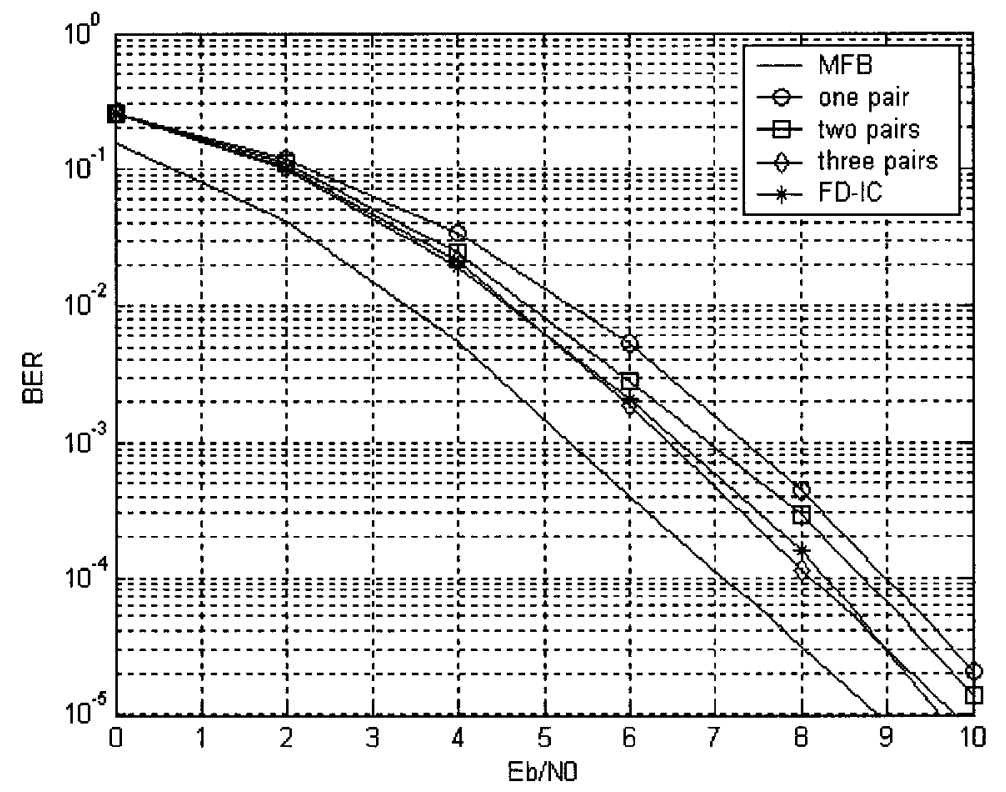

Figure 4-30. BER of the hybrid IC for QPSK over the 10-tap fading channel.

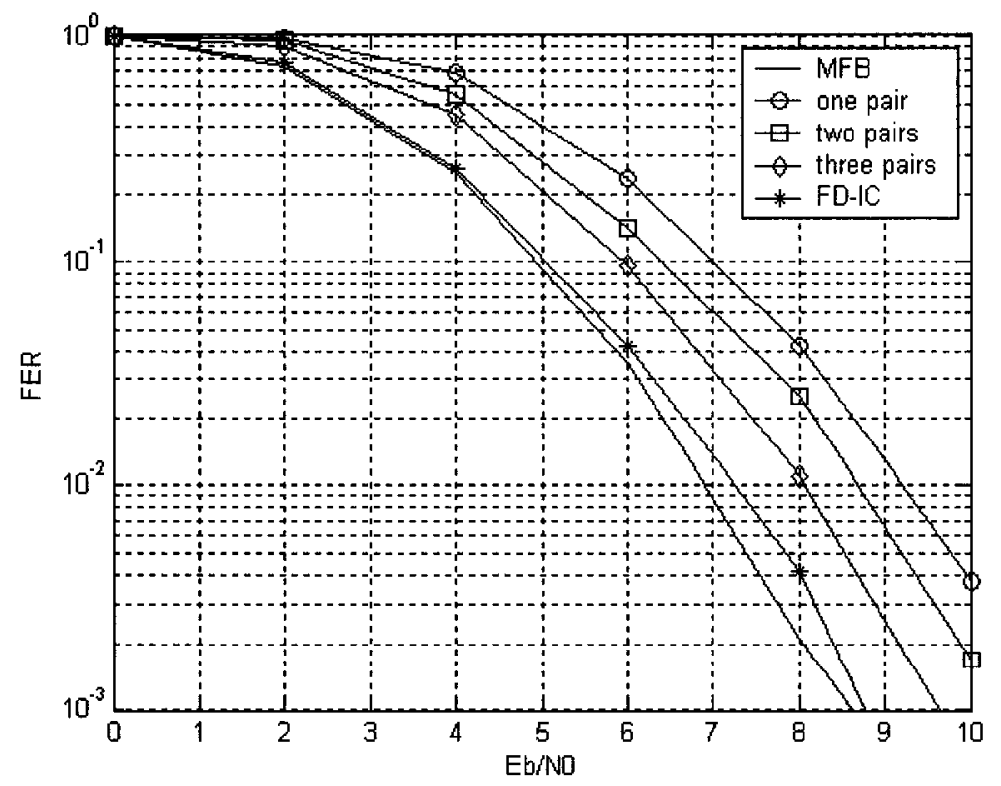

Figure 4-31. FER of the hybrid IC for QPSK over the 10-tap fading channel. 


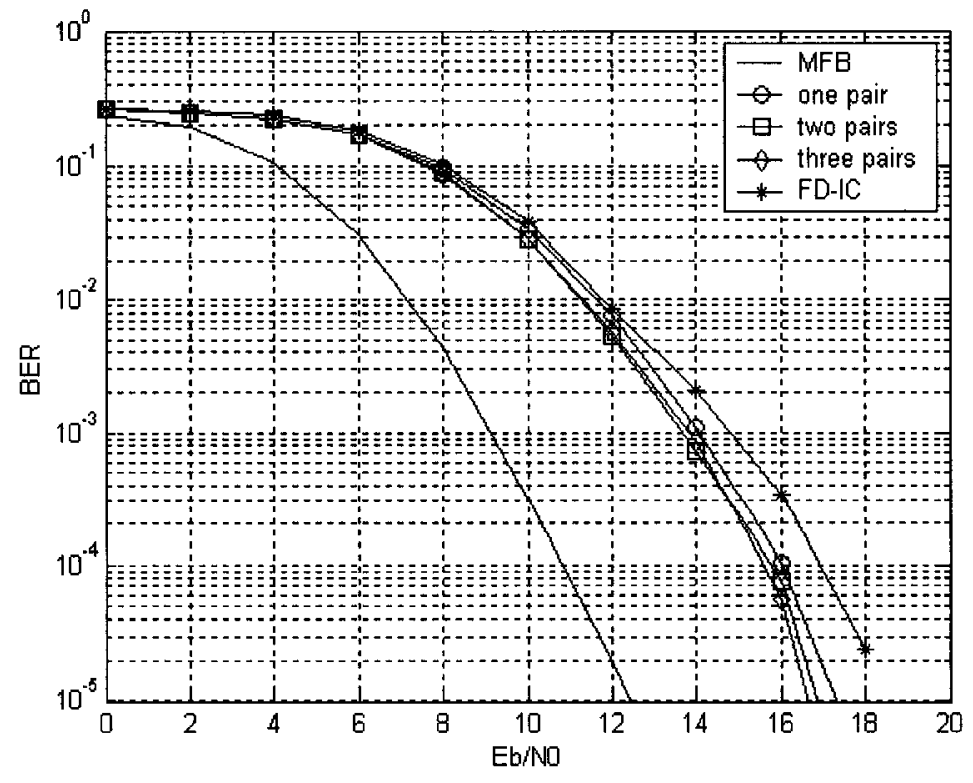

Figure 4-32. BER of the hybrid IC for 16-QAM TCM over the 10-tap fading channel.

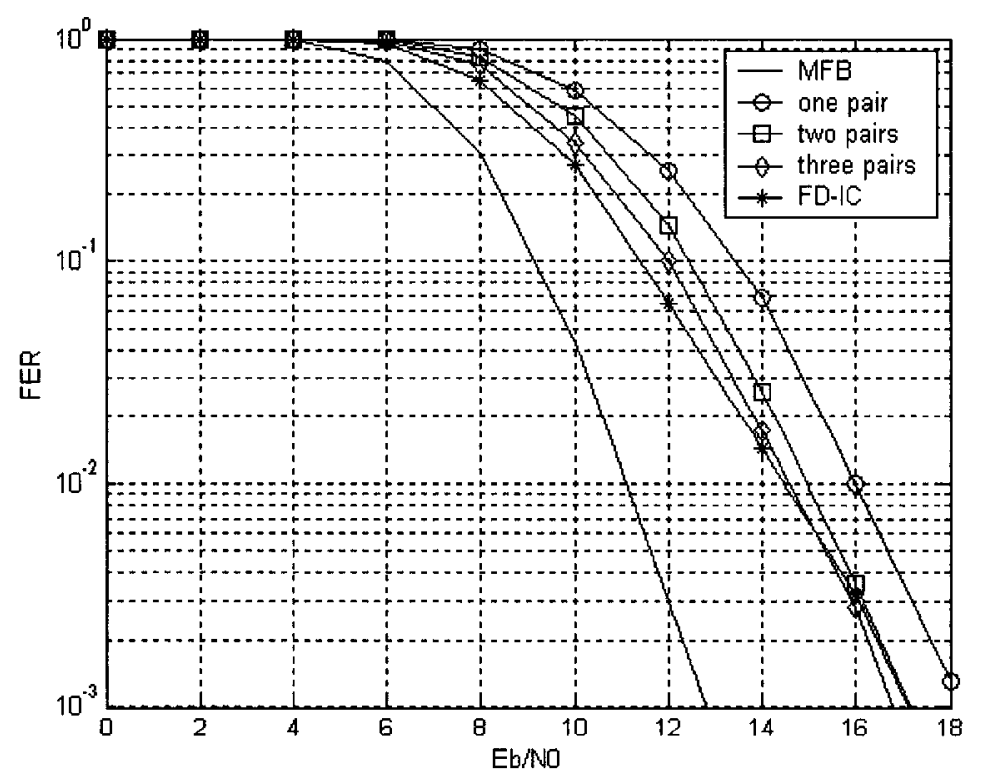

Figure 4-33. FER of the hybrid IC for 16-QAM TCM over the 10-tap fading channel. 


\section{Chapter 5}

\section{CONCLUSIONS AND FUTURE RESEARCH}

\subsection{Summary and Discussion}

The work in this thesis has investigated the performance of IC-based turbo equalization in the frequency domain over static and fading ISI channels in terms of the BER and FER. A hybrid reduced complexity IC has been proposed and its performances and computational complexity compared with that of TD-IC and FD-IC.

The FD-IC can improve the BER and FER performance over static ISI channels. For the long delay spread channel, the TD-IC requires prohibitively high complexity but the FD-IC can perform well and with low complexity.

For frequency selective fading channel, the BER performance of the FD-IC shows that turbo equalization does not give the same dramatic improvement over the SUI-5 and SUI-6 channels. However, the FER performance of FD-IC presents pretty good behaviour over these two channels. For the 10-taps fading channel, after the third iteration, the BER performance will become worse and worse, but FER still performs well. It can be found that although for a few message blocks the performance may be very poor due to the fading channel, causing the overall BER to be bad, the number of frame errors gets less by the iterative process. The FD-IC consists of two filters, the $P$ filter, and the $Q$-filter. The $P$-filter enhances the SNR and the $Q$-filter eliminates the ISI. However, if there are many errors from the previous iteration, instead of 
eliminating the ISI, the $Q$-filter will actually introduce more, causing error propagation. Therefore, the FD-IC turbo equalization works well for FER over fading channels.

It is known that ISI not only spreads out the signal energy but also adds the interference in the symbols. Fortunately, the proposed hybrid IC can effectively solve the problem when the powers in a few channel taps are dominant. The $P$-filter in the hybrid scheme not only takes on the same task as in the FD-IC but also eliminates the ISI that the $q$-filter cannot remove. When only a small number of taps are used in the $q$ filter, the $P$-filter does more of the work in suppressing the ISI so the hybrid is less susceptible to error propagation. On the other hand, when the number of taps is small, the hybrid IC is more like a linear equalizer, and therefore less effective at suppressing the interference. These two contradictory forces govern the performance of the hybrid IC. These issues can be seen clearly from the simulation results of the Proakis B and C channels. For the Proakis B channel, the hybrid scheme can achieve the same performance as the FD-IC. For the Proakis C channel, the hybrid scheme with two pairs can perform even better than the FD-IC. So, the hybrid scheme can work well over the static channels. For the fading channels, the BER performance of hybrid scheme performs differently depending on the channel and modulation scheme. For the SUI-5 channel, the BER and the FER performances do not improve after the third iteration. The BER degradation of the hybrid IC is very small compared with the FD-IC, but the FER performance of the hybrid IC cannot reach the MFB and so is a little bit worse than the FD-IC. For the 10-tap fading channel, the hybrid scheme for QPSK and 16QAM TCM can work well for FER but not for BER. The BER will work reasonably well as the number of pairs increase. However, in the meantime, some frame of 
message block may have large or very small bit errors so that the average bit error will not be determined as FER. Although in some situation, the BER cannot improve much, but the FER can perform well even in the SUI channel. When the FER is more concerned, the turbo equalization can improve the performance dramatically.

This hybrid scheme is aimed on further reducing the complexity. Since the characteristics of frequency domain make the computational complexity lower than in the time domain, especially for longer channel delay spreads, the comparison of the computational complexity of the TD-IC, FD-IC and the hybrid scheme shows that the proposed hybrid IC can have lower complexity than both TD-IC and FD-IC.

\subsection{Further Research}

The transmission systems investigated so far assume that the channel impulse response is perfectly known as is the noise variance. In practice, the channel impulse response and the noise variance are seldom known in advance and are often time varying. One solution is to employ adaptive schemes to estimate the channel impulse response and the noise variance, for instance, based on transmitting training sequences. In the first iteration, the training data can be used to estimate the initial coefficients of the LE. In the following iteration, the coefficients in the IC will be updated by adaptive techniques.

In the further research, turbo codes can be considered instead of convolutional code. Since in the no-ISI circumstances, turbo coding has shown to provide near Shannon-limit error correcting performance. Therefore, the use of turbo codes with turbo equalization may achieve better performance. 
In this thesis, the delay of interleaver and the IC are ignored. In practice, these delays will affect the whole system performance, especially for long message blocks. This will be another factor that further research will take account of.

Finally, the proposed model with various system parameter combinations over more complicated time-variant channels will perform. 


\section{LIST OF REFERENCES}

[1] C. Berrou, A. Glavieux and P. Thitimajshima, "Near Shannon limit errorcorrecting coding and decoding: Turbo-codes", IEEE International Conference on Communication (ICC), Geneva, Switzerland, May 1993, pp. 1064-1070.

[2] B. Sklar, "A primer on turbo code concepts", IEEE Communications Magazine, vol. 35, pp. 94-102, Dec. 1997.

[3] W. E. Ryan, $A$ Turbo Code Tutorial, http://cwc.ucsd.edu/ htullber/Text/tc_tutorial.pdf

[4] J. P. Woodard and L. Hanzo, "Comparative study of turbo decoding techniques: An overview", IEEE Transactions on Vehicular Technology, vol. 49, no. 6, pp. 2308-2233, November 2000.

[5] G. Bauch and V. Franz, "A comparison of soft-in/soft-out algorithms for turbodetection", International Conference on Telecommunication (ICT'98), Porto Carras, Greece, June 1998, pp. 259-263.

[6] J. Hagenauer "The turbo principle: Tutorial introduction and state of the art", International Symposium on Turbo Codes, Brest, France, Sept. 1997, pp. 1-11.

[7] C. Douillard, M. Jezequel, C. Berrou, A. Picart, P. Didier, A. Glavieux, "Iterative correction of intersymbol interference: Turbo-equalization", European Transactions on Telecommunications, vol. 6, June 1995, pp. 507-511.

[8] J. G. Proakis, Digital Communication, McGraw-Hill, 4th edition, 2001.

[9] S. H. Qureshi, "Adaptive equalization", Proceedings of the IEEE, vol. 73, no. 9, pp.1349-1387, Sept. 1985.

[10] D. A. George, R. Bowen and J. R. Storey, "An adaptive decision feedback equalizer", IEEE Transactions of Communication Technology, vol. 19, no. 3, pp. 281-293, June 1971.

[11] J. II Park and Y. Choi, "Turbo equalization for wireless cellular systems", IEICE Trans. Fundamentals, vol. E83-A, no.6, pp. 1184-1185, June 2000.

[12] D. Reynolds and X. Wang, " Low complexity turbo-equalization for diversity channels”, Signal Processing, vol. 81, no. 5, pp. 989-995, May 2001. 
[13] P. Magniez and P. Duhamel, "Turbo-equalization applied to trellis-coded modulations", IEEE Vehicular Technology Conf., Amsterdam, The Netherlands, Sept. 1999, pp. 2556-2560.

[14] X. Li and J. A. Ritcey, "Bit-interleaved coded modulation with iterative decoding using soft feedback", Electronics Letters, vol. 34, no. 10, pp. 942-943, $14^{\text {th }}$ May 1998.

[15] I. D. Marsland, P.T. Mathiopoulos, and S. Kallel, "Noncoherent turbo equalization for frequency selective Rayleigh fast fading channels", International Symposium on Turbo Codes, Brest, France, Sept. 1997, pp. 196-199.

[16] M. Tuchler, R. Kotter, A. Singer, "Turbo equalization: Principles and new results", IEEE Trans. on Communications, vol. 50, pp. 754-767, May 2002.

[17] A. Glavieux, C. Laot and J. Labat, "Turbo equalization over a frequency selective channel", International Symposium on Turbo Codes, Brest, France, Sept. 1997, pp. 96-102.

[18] A. Glavieux, C. Laot and J. Labat, "Turbo equalization: adaptive equalization and channel decoding jointly optimized", IEEE Journal on Selected Areas of Communications, vol. 19, pp. 1744-1752, Sept. 2001.

[19] I. Fijalkow, A. Roumy, S. Ronger, D. Pirez and P. Vila, "Improved interference cancellation for turbo-equalization", IEEE International Conference on Acoustics, Speech and Signal Processing (ICASSP), Turkey, June 2000, pp. 416419.

[20] X. Wang and H. Vincent Poor, "Iterative (turbo) soft interference cancellation and decoding for coded CDMA", IEEE Transaction on Communications, vol.47, no. 7, pp. 1046-1061, July 1999.

[21] M. Tuchler and J. Hagenauer, "Turbo equalization using frequency domain equalizers", Proc. Allerton Conf., Monticello, IL, USA, Oct 2000.

[22] M. Tuchler and J. Hagenauer, "Linear time and frequency domain turbo equalization", IEEE Vehicular Technology Conf., Rhodes, Greece, May 2001, pp. 1449-1453.

[23] N. Lin, "A Comparison of Turbo Equalization Techniques for Stationary Intersymbol Interference Channels", Master's thesis, Carleton University, 2002.

[24] R. W. Chang, and J. C. Hancock, "On receiver structures for channels having memory", IEEE Transactions on Information Theory, vol.12, pp.463-468, Oct. 1966. 
[25] L. R. Bahl, J. Cocke, F. Jelinek and J. Raviv, “ Optimal decoding of linear codes for minimizing symbol error rate", IEEE Transactions on Information Theory, vol. IT-20, pp. 284-287, March 1974.

[26] H. Sari, G. Karam, and I. Jeanclaude, "Frequency-domain equalization of mobile radio and terrestrial broadcast channels", Globecom, San Francisco, 1995, pp. 15.

[27] K. Berberidis, "A frequency-domain decision feedback equalizer for multipath echo cancellation", Proc. Globecom '95, Singapore, December 1995, pp. 98-102.

[28] M. V. Clark, "Adaptive frequency-domain equalization and diversity combining for broadband wireless communications", IEEE Journal on Selected Areas in Communications, vol.16, No. 8, pp. 1385-1395, October 1998.

[29] D. Falconer, L. Ariyavisitakul, A. Benyamin-Seeyar and B. Eidson, "White paper: Single carrier modulation scheme with frequency domain equalization for broadband wireless systems",

http://www.sce.carleton.ca/bbw/papers/Ariyavisitakul.pdf

[30] V. Erceg, K. K.S. Hari, M. S. Smith, K. P. Sheikh, C. Tappenden, J. M. Costa, D. S. Baum and C. Bushue, "Channel models for fixed wireless applications", IEEE 802.16. Proposal 802.16.3c-01/29r4.

[31] G. Ungerboeck, "Channel Coding with Multilever/Phase Signal ", IEEE Trans. Information Theory, vol. 28, no. 1, pp. 55-66, Jan. 1982. 


\section{Appendix A Coefficients, Mean and Variance for the Original IC Turbo Equalization}

\section{A.1 Coefficients of the original IC}

The output of a time-domain IC is from Equation (3-1) [17]

$$
\begin{aligned}
z_{n} & =\sum_{k=-L}^{0} p_{k} r_{n-k}-\sum_{\substack{k=-L \\
k \neq 0}}^{L} q_{k} v_{n-k} \\
& =\sum_{k=-L}^{0} p_{k} r_{n-k}-\sum_{k=-L}^{L} q_{k} v_{n-k}+q_{0} v_{n} .
\end{aligned}
$$

A corresponding frequency domain IC would have output given by

$$
z_{n}=\frac{1}{N} \sum_{k=0}^{N-1}\left(P_{k} R_{k}-Q_{k} V_{k}\right) e^{j 2 \pi k n / N}+q_{0} v_{n},
$$

where, from the definition of the DFT,

$$
q_{0}=\frac{1}{N} \sum_{k=0}^{N-1} Q_{k}
$$

and $N$ is the length of the DFT block. The optimum filter coefficients are obtained according to the minimum mean square error criterion. The mean square error is

$$
J=E\left[\left|z_{n}-v_{n}\right|^{2}\right]
$$

and the coefficients are found by solving the set of linear equations arising when the partial derivating of $J$ with respect to $P_{l}$ and $Q_{l}$ are set to zero.

\section{A.1.1 Coefficients of $Q$-filter}

$$
\begin{aligned}
& \text { Setting } \frac{\partial J}{\partial Q_{l}}=0 \text { is equivalent to setting } \\
& E\left[\left(z_{n}-v_{n}\right) \frac{\partial}{\partial Q_{l}}\left(z_{n}-v_{n}\right)^{*}\right]=0 .
\end{aligned}
$$


Since

$$
\begin{aligned}
\frac{\partial}{\partial Q_{l}}\left(z_{n}-v_{n}\right) & =\frac{1}{N} \sum_{k=0}^{N-1} \frac{\partial}{\partial Q_{l}}\left(P_{k} R_{k}-Q_{k} V_{k}\right) e^{j 2 \pi k n / N}+v_{n} \sum_{k=0}^{N-1} \frac{\partial q_{0}}{\partial Q_{l}} \\
& =-\frac{1}{N} V_{l} e^{j 2 m n l / N}+\frac{1}{N} v_{n},
\end{aligned}
$$

this is equivalent to

$$
E\left[\left(z_{n}-v_{n}\right)\left(-\frac{1}{N} V_{l}^{*} e^{-j 2 m l / N}+\frac{1}{N} v_{n}^{*}\right)\right]=0
$$

or

$$
E\left[\left(z_{n}-v_{n}\right) V_{l}^{*}\right] e^{-j 2 m l / N}=E\left[\left(z_{n}-v_{n}\right) v_{n}^{*}\right]
$$

The left-hand side of Equation (A-8) is

$$
\begin{aligned}
& E\left[\left(z_{n}-v_{n}\right) V_{l}^{*}\right] e^{-j 2 \pi n l / N} \\
& =E\left[\left(\frac{1}{N} \sum_{k=0}^{N-1}\left(P_{k} R_{k}-Q_{k} V_{k}\right) e^{j 2 \pi k n / N}+q_{0} v_{n}-v_{n}\right) V_{l}^{*}\right] e^{-j 2 \pi n l / N} \\
& =\frac{1}{N} \sum_{k=0}^{N-1}\left(P_{k} E\left[R_{k} V_{l}^{*}\right]-Q_{k} E\left[V_{k} V_{l}^{*}\right]\right) e^{j 2 \pi(k-l) n / N}+\left(q_{0}-1\right) E\left[v_{n} V_{l}^{*}\right] e^{-j 2 \pi n l / N} .
\end{aligned}
$$

Since $R_{k}=H_{k} V_{k}+W_{k}, V_{l}=\sum_{m=0}^{N-1} v_{m} e^{-j 2 \pi m / N}$ and $E\left[W_{k}\right]=0$, this becomes

$$
\begin{aligned}
& E\left[\left(z_{n}-v_{n}\right) V_{l}^{*}\right] e^{-j 2 \pi n l / N} \\
& \quad=\frac{1}{N} \sum_{k=0}^{N-1}\left(P_{k} H_{k}-Q_{k}\right) E\left[V_{k} V_{l}^{*}\right] e^{j 2 \pi(k-l) n / N}+\left(q_{0}-1\right) \sum_{m=0}^{N-1} E\left[v_{n} v_{m}^{*}\right] e^{j 2 \pi(m-n) / N}
\end{aligned}
$$

Since $E\left[v_{n} v_{m}^{*}\right]=\sigma_{v}^{2} \delta_{n-m}$ and $E\left[V_{k} V_{l}^{*}\right]=\sigma_{v}^{2} N \delta_{k-l}$, this becomes

$$
E\left[\left(z_{n}-v_{n}\right) V_{l}^{*}\right] e^{-j 2 \pi n l / N}
$$




$$
\begin{aligned}
& =\frac{1}{N} \sum_{k=0}^{N-1} \sigma_{v}^{2} N \delta_{k-l}\left(P_{k} H_{k}-Q_{k}\right) e^{j 2 \pi(k-l) n / N}+\left(q_{0}-1\right) \sum_{m=0}^{N-1} \sigma_{v}^{2} \delta_{m-n} e^{j 2 \pi l(m-n) / N} \\
& =\sigma_{v}^{2}\left(P_{l} H_{l}-Q_{l}\right)+\sigma_{v}^{2}\left(q_{0}-1\right) .
\end{aligned}
$$

The right-hand side of Equation (A-8) is

$$
\begin{aligned}
E\left[\left(z_{n}-v_{n}\right) v_{n}^{*}\right] & =E\left[\left[\sum_{k=0}^{N-1} \frac{1}{N}\left(P_{k} R_{k}-Q_{k} V_{k}\right) e^{j 2 \pi k n / N}+\left(q_{0}-1\right) v_{n}\right] v_{n}^{*}\right] \\
& =\frac{1}{N}\left[\sum_{k=0}^{N-1}\left(P_{k} E\left[R_{k} v_{n}^{*}\right]-Q_{k} E\left[V_{k} v_{n}^{*}\right] e^{j 2 \pi k n / N}\right]+\left(q_{0}-1\right) E\left[\left|v_{n}\right|^{2}\right]\right. \\
& =\frac{1}{N}\left[\sum_{k=0}^{N-1}\left(P_{k} H_{k}-Q_{k}\right) E\left[V_{k} v_{n}^{*}\right] e^{j 2 \pi k n / N}\right]+\left(q_{0}-1\right) \sigma_{v}^{2} .
\end{aligned}
$$

Since $E\left[V_{k} v_{n}^{*}\right]=\sigma_{v}^{2} e^{-j 2 \pi k n / N}$, this becomes

$$
\begin{aligned}
E\left[\left(z_{n}-v_{n}\right) v_{n}^{*}\right] & =\frac{1}{N} \sum_{k=0}^{N-1}\left(P_{k} H_{k}-Q_{k}\right) \sigma_{v}^{2}+\left(q_{0}-1\right) \sigma_{v}^{2} \\
& =\sigma_{v}^{2}\left[\frac{1}{N} \sum_{k=0}^{N-1} P_{k} H_{k}-\frac{1}{N} \sum_{k=0}^{N-1} Q_{k}+\left(q_{0}-1\right)\right] \\
& =\sigma_{v}^{2}\left[\frac{1}{N} \sum_{k=0}^{N-1} P_{k} H_{k}-q_{0}+\left(q_{0}-1\right)\right] \\
& =\sigma_{v}^{2}\left[\frac{1}{N} \sum_{k=0}^{N-1} P_{k} H_{k}-1\right] .
\end{aligned}
$$

Equating Equation (A-11) to Equation (A-13) gives

$$
\sigma_{v}^{2}\left(P_{l} H_{l}-Q_{l}\right)+\sigma_{v}^{2}\left(q_{0}-1\right)=\sigma_{v}^{2}\left[\frac{1}{N} \sum_{k=0}^{N-1} P_{k} H_{k}-1\right]
$$

or

$$
Q_{l}-q_{0}=P_{l} H_{l}-\frac{1}{N} \sum_{k=0}^{N-1} P_{k} H_{k}
$$


or

$$
Q_{l}-\frac{1}{N} \sum_{k=0}^{N-1} Q_{k}=P_{l} H_{l}-\frac{1}{N} \sum_{k=0}^{N-1} P_{k} H_{k}
$$

So, a valid solution is

$$
Q_{l}=P_{l} H_{l}
$$

\section{A.1.2 Coefficients of $\boldsymbol{P}$-filter}

$$
\begin{aligned}
& \text { Setting } \frac{\partial J}{\partial P_{l}}=0 \text { is equivalent to } \\
& E\left[\left(z_{n}-v_{n}\right) \frac{\partial}{\partial P_{l}}\left(z_{n}-v_{n}\right)^{*}\right]=0 .
\end{aligned}
$$

Since

$$
\begin{aligned}
\frac{\partial}{\partial P_{l}}\left(z_{n}-v_{n}\right) & =\frac{1}{N} \sum_{k=0}^{N-1} \frac{\partial}{\partial P_{l}}\left(P_{k} R_{k}-Q_{k} V_{k}\right) e^{j 2 \pi k n / N} \\
& =\frac{1}{N} \sum_{k=0}^{N-1} R_{k} \delta_{k-l} e^{j 2 \pi k n / N} \\
& =\frac{1}{N} R_{l} e^{j 2 \pi n l / N},
\end{aligned}
$$

this is equivalent to

$$
E\left[\left(z_{n}-v_{n}\right) \frac{1}{N} R_{l}^{*} e^{-j 2 \pi n l / N}\right]=0
$$

or

$$
E\left[z_{n} R_{l}^{*}\right]=E\left[v_{n} R_{l}^{*}\right]
$$

The left-hand side of (A-18) is 


$$
\begin{aligned}
E\left[z_{n} R_{l}^{*}\right]= & E\left[\frac{1}{N} \sum_{k=0}^{N-1}\left(P_{k} R_{k}-Q_{k} V_{k}\right) e^{j 2 \pi k n / N} R_{l}^{*}\right]+q_{0} E\left[v_{n} R_{l}^{*}\right] \\
= & \frac{1}{N} \sum_{k=0}^{N-1}\left(P_{k} E\left[R_{k} R_{l}^{*}\right]-Q_{k} E\left[V_{k} R_{l}^{*}\right]\right) e^{j 2 \pi k n / N}+q_{0} E\left[v_{n}\left(H_{l}^{*} V_{l}^{*}+W_{l}^{*}\right)\right] \\
= & \frac{1}{N} \sum_{k=0}^{N-1}\left(P_{k} E\left[\left(H_{k} V_{k}+W_{k}\right)\left(H_{l}^{*} V_{l}^{*}+W_{l}^{*}\right)\right]-Q_{k} E\left[V_{k}\left(H_{l}^{*} V_{l}^{*}+W_{l}^{*}\right)\right]\right) e^{j 2 \pi k n / N} \\
& \quad+q_{0} H_{l}^{*} E\left[v_{n} V_{l}^{*}\right] \\
= & \frac{1}{N} \sum_{k=0}^{N-1}\left[P_{k}\left(H_{k} H_{l}^{*} E\left[V_{k} V_{l}^{*}\right]+E\left[W_{k} W_{l}^{*}\right]\right)-Q_{k} H_{l}^{*} E\left[V_{k} V_{l}^{*}\right]\right] e^{j 2 \pi k n / N} \\
& \quad+q_{0} H_{l}^{*} E\left[v_{n} V_{l}^{*}\right]
\end{aligned}
$$

Since $E\left[V_{k} V_{l}^{*}\right]=\sigma_{v}^{2} N \delta_{k-l}$ and $E\left[W_{k} W_{l}^{*}\right]=N_{0} N \delta_{k-l}$, this becomes

$$
\begin{aligned}
E\left[z_{n} R_{l}^{*}\right]= & \frac{1}{N} \sum_{k=0}^{N-1}\left[P_{l}\left(H_{l} H_{l}^{*} \sigma_{v}^{2}+N_{0}\right) N \delta_{k-l}-Q_{k} H_{l}^{*} \sigma_{v}^{2} N \delta_{k-l}\right] e^{j 2 \pi k n / N} \\
& +q_{0} H_{l}^{*} \sigma_{v}^{2} e^{j 2 \pi n l / N} \\
= & {\left[P_{l}\left(\left|H_{l}\right|^{2} \sigma_{v}^{2}+N_{0}\right)-Q_{l} H_{l}^{*} \sigma_{v}^{2}+q_{0} H_{l}^{*} \sigma_{v}^{2}\right] e^{j 2 \pi n l / N} . }
\end{aligned}
$$

The right-hand side of Equation (A-18) is

$$
\begin{aligned}
E\left[v_{n} R_{l}^{*}\right] & =H_{l}^{*} E\left[v_{n} V_{l}^{*}\right]+E\left[v_{n} W_{l}^{*}\right] \\
& =H_{l}^{*} \sigma_{v}^{2} e^{j 2 \pi n l / N} .
\end{aligned}
$$

Combining Equation (A-20) and (A-21) gives

$$
\left[P_{l}\left(\left|H_{l}\right|^{2} \sigma_{v}^{2}+N_{0}\right)-Q_{l} H_{l}^{*} \sigma_{v}^{2}+q_{0} H_{l}^{*} \sigma_{v}^{2}\right] e^{j 2 \pi n l / N}=H_{l}^{*} \sigma_{v}^{2} e^{j 2 \pi n l / N} .
$$

Substituting $Q_{l}=P_{l} H_{l}$ gives

$$
P_{l} N_{0}+q_{0} H_{l}^{*} \sigma_{v}^{2}=H_{l}^{*} \sigma_{v}^{2}
$$

or 


$$
P_{l}=\frac{\sigma_{v}^{2} H_{l}^{*}}{N_{0}}\left(1-q_{0}\right)
$$

However

$$
\begin{aligned}
q_{0} & =\frac{1}{N} \sum_{l=0}^{N-1} Q_{l}=\frac{1}{N} \sum_{l=0}^{N-1} P_{l} H_{l} \\
& =\frac{\sigma_{v}^{2}}{N_{0}}\left(1-q_{0}\right) \frac{1}{N} \sum_{l=0}^{N-1}\left|H_{l}\right|^{2} .
\end{aligned}
$$

Define

$$
E_{h}=\frac{1}{N} \sum_{l=0}^{N-1}\left|H_{l}\right|^{2}
$$

as the overall channel attenuation, so

$$
q_{0}=\frac{\frac{\sigma_{v}^{2}}{N_{0}} E_{h}}{\frac{\sigma_{v}^{2}}{N_{0}} E_{h}+1}=\frac{\sigma_{v}^{2} E_{h}}{\sigma_{v}^{2} E_{h}+N_{0}} .
$$

Then

$$
P_{l}=\frac{\sigma_{v}^{2} H_{l}^{*}}{\sigma_{v}^{2} E_{h}+N_{0}}
$$

and

$$
Q_{l}=\frac{\sigma_{v}^{2}\left|H_{l}\right|^{2}}{\sigma_{v}^{2} E_{h}+N_{0}} .
$$

\section{A.2 Mean and Variance}

To find the mean and variance of $z_{n}$, conditioned on $v_{n}=v$, note that since $Q_{l}=P_{l} H_{l}$ and $R_{l}=H_{l} V_{l}+W_{l}$, 


$$
P_{l} R_{l}-Q_{l} V_{l}=P_{l}\left(H_{l} V_{l}+W_{l}\right)-\left(P_{l} H_{l}\right) V_{l}=P_{l} W_{l}
$$

so,

$$
z_{n}=\frac{1}{N} \sum_{k=0}^{N-1} P_{l} W_{l} e^{j 2 \pi k n / N}+q_{0} v_{n}
$$

The mean is therefore

$$
\mu_{z}(v)=E\left[z_{n} \mid v_{n}=v\right]=q_{0} v
$$

The variance is

$$
\begin{aligned}
\sigma_{z}^{2} & =E\left[\left|z_{n}\right|^{2} \mid v_{n}=v\right]-\left|\mu_{z}(v)\right|^{2} \\
& =E\left[\left|\frac{1}{N} \sum_{k=0}^{N-1} P_{k} W_{k} e^{j 2 \pi k n / N}\right|^{2}\right]+\left.\left|q_{0}\right|^{2}\left|E\left[v_{n} \mid v_{n}=v\right]^{2}-\right| q_{0}\right|^{2}|v|^{2} \mid \\
& =\frac{1}{N^{2}} \sum_{k=0}^{N-1} \sum_{l=0}^{N-1} P_{k} P_{l}^{*} E\left[W_{k} W_{l}^{*}\right] e^{j 2 \pi(k-l) n / N} \\
& =\frac{1}{N^{2}} \sum_{k=0}^{N-1} \sum_{l=0}^{N-1} P_{k} P_{l}^{*} N_{0} N \delta_{k-l} e^{j 2 \pi(k-l) n / N} \\
& =N_{0} \frac{1}{N} \sum_{k=0}^{N-1}\left|P_{k}\right|^{2} \\
& =N_{0}\left(\frac{\sigma_{v}^{2}}{\sigma_{v}^{2} E_{h}+N_{0}}\right)^{2} \frac{1}{N} \sum_{k=0}^{N-1}\left|H_{k}\right|^{2} \\
& =\sigma_{v}^{2}\left(1-q_{0}\right) q_{0}, \\
& =N_{0}\left(\frac{\sigma_{v}^{2}}{\sigma_{v}^{2} E_{h}+N_{0}}\right)\left(\frac{\sigma_{v}^{2} E_{h}}{\sigma_{v}^{2} E_{h}+N_{0}}\right)
\end{aligned}
$$

where $q_{0}=\frac{\sigma_{v}^{2} E_{h}}{\sigma_{v}^{2} E_{h}+N_{0}}$. 


\section{Appendix B Coefficients, Mean and Variance for Hybrid Reduced Complexity Turbo Equalization}

\section{B.1 Coefficients of the Hybrid IC}

The output of the hybrid IC is

$$
z_{n}=\frac{1}{N} \sum_{k=0}^{N-1} P_{k} R_{k} e^{j 2 \pi k n / N}-\sum_{i=1}^{N_{t}}\left(q_{M_{i}} v_{n-M_{i}}+q_{-M_{i}} v_{n+M_{i}}\right)
$$

where, $\left\{M_{i} \mid i \in\left\{1,2, \cdots N_{t}\right\}\right\}$, are the indices of the desired feedback taps. The MMSE is used to determine the optimum coefficients. The mean square error is

$$
J=E\left[\left|z_{n}-v_{n}\right|^{2}\right],
$$

and the coefficients are determined by solving the set of linear equations when the partial derivating of $J$ with respect to $P_{l}$ and $q_{m}$ are set to zero.

\section{B.1.1 $P$-filter coefficients}

$$
\begin{aligned}
& \text { Setting } \frac{\partial J}{\partial P_{l}}=0 \text { is equivalent to } \\
& E\left[\left(z_{n}-v_{n}\right) \frac{\partial}{\partial P_{l}}\left(\left(z_{n}-v_{n}\right)^{*}\right)\right]=0 .
\end{aligned}
$$

Since from $(\mathrm{A}-17)$

$$
\frac{\partial}{\partial P_{l}}\left(z_{n}-v_{n}\right)=\frac{1}{N} R_{l} e^{j 2 \pi n l / N},
$$

this is equivalent to

$$
E\left[\left(z_{n}-v_{n}\right) \frac{1}{N} R_{l}^{*} e^{-j 2 m l / N}\right]=0
$$

or 


$$
E\left[z_{n} R_{l}^{*}\right]=E\left[v_{n} R_{l}^{*}\right]
$$

The left-hand side of Equation (B-6) is

$$
E\left[z_{n} R_{l}^{*}\right]=E\left[\sum_{k=0}^{N-1} \frac{1}{N} P_{k} R_{k} R_{l}^{*} e^{j 2 \pi t n / N}-\sum_{i=1}^{N_{t}} q_{M_{i}} v_{n-M_{i}} R_{l}^{*}-\sum_{i=1}^{N_{t}} q_{-M_{i}} v_{n+M_{i}} R_{l}^{*}\right] .
$$

The first term of Equation (B-7) is

$$
\begin{aligned}
E\left[\sum_{k=0}^{N-1} \frac{1}{N} P_{k} R_{k} R_{l}^{*} e^{j 2 \pi k n / N}\right]=E\left[\sum_{k=0}^{N-1} \frac{1}{N} P_{k}\left(H_{k} V_{k}+W_{k}\right)\left(H_{l}^{*} V_{l}^{*}+W_{l}^{*}\right) e^{j 2 \pi k n / N}\right] \\
=\frac{1}{N} \sum_{k=0}^{N-1} P_{k}\left(H_{k} H_{l}^{*} E\left[V_{k} V_{l}^{*}\right]+E\left[W_{k} W_{l}^{*}\right]\right) e^{j 2 \pi k n / N} \\
=\frac{1}{N} \sum_{k=0}^{N-1} P_{k}\left(H_{k} H_{l}^{*} \sigma_{v}^{2} N \delta_{k-l}+N_{0} N \delta_{k-l}\right) e^{j 2 \pi k n / N} \\
=P_{l}\left(H_{l} H_{l}^{*} \sigma_{v}^{2}+N_{0}\right) e^{j 2 \pi l / N}
\end{aligned}
$$

The second term of Equation (B-7) is

$$
\begin{gathered}
E\left[\sum_{i=1}^{N_{t}} q_{M_{i}} v_{n-M_{i}} R_{l}^{*}\right]=E\left[\left(\sum_{i=1}^{N_{t}} q_{M_{i}}\left(\frac{1}{N} \sum_{k=0}^{N-1} V_{k} e^{j 2 \pi\left(n-M_{i}\right) k / N}\right)\left(H_{l}^{*} V_{l}^{*}+W_{l}^{*}\right)\right]\right. \\
=\frac{1}{N} \sum_{i=1}^{N_{i}} q_{M_{i}} \sum_{k=0}^{N-1}\left(H_{l}^{*} E\left[V_{k} V_{l}^{*}\right]+E\left[V_{k} W_{l}^{*}\right] e^{j 2 \pi\left(n-M_{i}\right) k / N}\right. \\
=\frac{1}{N} \sum_{i=1}^{N_{i}} q_{M_{i}} \sum_{k=0}^{N-1} H_{i}^{*} N \sigma_{v}^{2} \delta_{k-l} e^{j 2 \pi\left(n-M_{i}\right) k / N} \\
=H_{l}^{*} \sigma_{v}^{2} e^{j 2 \pi n l / N} \sum_{i=1}^{N_{i}} q_{M_{i}} e^{-j 2 \pi M_{l} / / N} .
\end{gathered}
$$

The third term of Equation (B-7) is

$$
E\left[\sum_{i=1}^{N_{t}} q_{-M_{i}} v_{n+M_{i}} R_{l}^{*}\right]=E\left[\left(\sum_{i=1}^{N_{4}} q_{-M_{i}}\left(\frac{1}{N} \sum_{k=0}^{N-1} V_{k} e^{j 2 \pi\left(n+M_{i}\right) k / N}\right)\left(H_{l}^{*} V_{l}^{*}+W_{l}^{*}\right)\right]\right.
$$




$$
\begin{aligned}
& =\frac{1}{N} \sum_{i=1}^{N_{t}} q_{-M_{i}} \sum_{k=0}^{N-1}\left(H_{l}^{*} E\left[V_{k} V_{l}^{*}\right]+E\left[V_{k} W_{l}^{*}\right]\right) e^{j 2 \pi\left(n+M_{i}\right) k / N} \\
& =\frac{1}{N} \sum_{i=1}^{N_{t}} q_{-M_{i}} \sum_{k=0}^{N-1} H_{l}^{*} N \sigma_{v}^{2} \delta_{k-l} e^{j 2 \pi\left(n+M_{i}\right) k / N} \\
& =H_{l}^{*} \sigma_{v}^{2} e^{j 2 \pi n l / N} \sum_{i=1}^{N_{t}} q_{-M_{i}} e^{j 2 \pi M_{i} / / N} .
\end{aligned}
$$

Therefore, Equation (B-7) is rewritten as

$$
\begin{aligned}
E\left[z_{n} R_{l}^{*}\right]=P_{l}\left(H_{l} H_{l}^{*} \sigma_{v}^{2}+N_{0}\right) e^{j 2 \pi n l / N}- \\
H_{l}^{*} \sigma_{v}^{2} e^{j 2 \pi n l / N} \sum_{i=1}^{N_{t}}\left(q_{M_{i}} e^{-j 2 \pi M_{i} l / N}+q_{-M_{i}} e^{j 2 \pi M M_{i} / N}\right) .
\end{aligned}
$$

And, the right-hand side of Equation (B-6) is

$$
\begin{aligned}
E\left[v_{n} R_{l}^{*}\right] & =\frac{1}{N} E\left[\sum_{k=0}^{N-1} V_{k} e^{j 2 \pi k n / N}\left(H_{l}^{*} V_{l}^{*}+W_{l}^{*}\right)\right] \\
& =\frac{1}{N}\left[\sum_{k=0}^{N-1} H_{l}^{*} E\left[V_{k} V_{l}^{*}\right] e^{j 2 \pi k n / N}\right] \\
& =\frac{1}{N}\left[\sum_{k=0}^{N-1} H_{l}^{*} \sigma_{v}^{2} N \delta_{k-l} e^{j 2 \pi k n / N}\right] \\
& =H_{l}^{*} \sigma_{v}^{2} e^{j 2 \pi n l / N} .
\end{aligned}
$$

Equating Equation (B-11) to Equation (B-12) gives

$$
P_{l}\left(\left|H_{l}\right|^{2} \sigma_{v}^{2}+N_{0}\right)-H_{l}^{*} \sigma_{v}^{2} \sum_{i=1}^{N_{t}}\left(q_{M_{i}} e^{-j 2 \pi M_{l} l / N}+q_{-M_{i}} e^{j 2 \pi M_{i} l / N}\right)=H_{l}^{*} \sigma_{v}^{2}
$$

or

$$
P_{l}=\frac{H_{l}^{*} \sigma_{v}^{2}}{\left|H_{l}\right|^{2} \sigma_{v}^{2}+N_{0}}\left[1+\sum_{i=1}^{N_{t}}\left(q_{M_{i}} e^{-j 2 \pi M_{i} l / N}+q_{-M_{i}} e^{j 2 \pi M_{i} l / N}\right)\right] .
$$




\section{B.1.2 $q$-filter coefficients}

Setting $\frac{\partial J}{\partial q_{m}}=0$ is equivalent to

$$
E\left[\left(z_{n}-v_{n}\right) \frac{\partial}{\partial q_{m}}\left(\left(z_{n}-v_{n}\right)^{*}\right)\right]=0
$$

Since

$$
\frac{\partial}{\partial q_{m}}\left(z_{n}-v_{n}\right)=-v_{n-m}, \text { for } m \in\left\{M_{1}, \cdots, M_{i}, \cdots, M_{N_{t}}\right\}
$$

this is equivalent to

$$
E\left[\left(z_{n}-v_{n}\right)\left(-v_{n-m}^{*}\right)\right]=0 .
$$

And for $m \in\left\{M_{1}, \cdots, M_{i}, \cdots, M_{N_{t}}\right\}$

$$
\begin{gathered}
E\left[\left(z_{n}-v_{n}\right)\left(-v_{n-m}^{*}\right)\right]=E\left[\left(\frac{1}{N} \sum_{k=0}^{N-1} P_{k} R_{k} e^{j 2 \pi k n / N}-\sum_{i=1}^{N_{t}}\left(q_{M_{i}} v_{n-M_{i}}+q_{-M_{i}} v_{n+M_{i}}\right)-v_{n}\right)\left(-v_{n-m}^{*}\right)\right] \\
=-E\left[\frac{1}{N} \sum_{k=0}^{N-1} P_{k} R_{k}\left(v_{n-m}^{*}\right) e^{j 2 \pi k n / N}\right]+E\left(\sum_{i=1}^{N_{t}}\left(q_{M_{i}} v_{n-M_{i}}+q_{-M_{i}} v_{n+M_{i}}\right) v_{n-m}^{*}\right) \\
=E\left[-\frac{1}{N} \sum_{k=0}^{N-1} P_{k} R_{k} v_{n-m}^{*} e^{j 2 \pi k n / N}\right]+q_{m} \sigma_{v}^{2}
\end{gathered}
$$

for $v_{n-m}=\frac{1}{N} \sum_{p=0}^{N-1} V_{p} e^{-j 2 \pi(n-m) p / N}$, then

$$
\begin{gathered}
E\left[\left(z_{n}-v_{n}\right)\left(-v_{n-m}^{*}\right)\right]=E\left[-\frac{1}{N^{2}} \sum_{k=0}^{N-1} P_{k}\left(H_{k} V_{k}+W_{k}\right) \sum_{p=0}^{N-1} V_{p}^{*} e^{-j 2 \pi(n-m) p / N} e^{j 2 \pi k n / N}\right]+q_{m} \sigma_{v}^{2} \\
=-\frac{1}{N^{2}} \sum_{k=0}^{N-1} \sum_{p=0}^{N-1} P_{k} H_{k} E\left[V_{k} V_{p}^{*}\right] e^{j 2 \pi n p / N} e^{j 2 \pi(k-p) n / N}+q_{m} \sigma_{v}^{2} \\
=-\frac{1}{N^{2}} \sum_{k=0}^{N-1} \sum_{p=0}^{N-1} P_{k} H_{k} N \sigma_{v}^{2} \delta_{k-p} e^{j 2 \pi m p / N} e^{j 2 \pi(k-p) n / N}+q_{m} \sigma_{v}^{2}
\end{gathered}
$$




$$
=-\sigma_{v}^{2} \frac{1}{N} \sum_{k=0}^{N-1} P_{k} H_{k} e^{j 2 m n k / N}+q_{m} \sigma_{v}^{2}=0,
$$

so, for $m \in\left\{M_{1}, \cdots, M_{i}, \cdots, M_{N_{t}}\right\}$

$$
q_{m}=\frac{1}{N} \sum_{k=0}^{N-1} P_{k} H_{k} e^{j 2 \pi k m / N}
$$

Similarly,

$$
q_{-m}=\frac{1}{N} \sum_{k=0}^{N-1} P_{k} H_{k} e^{-j 2 \pi k m / N} .
$$

Substituting Equation (B-13) into Equation (B-19) gives, for

$$
\begin{aligned}
m \in\left\{M_{1}, \cdots,\right. & \left.M_{i}, \cdots, M_{N_{t}}\right\} \\
q_{m}= & \frac{1}{N} \sum_{k=0}^{N-1} \frac{H_{k}^{*} \sigma_{v}^{2}}{\left|H_{k}\right|^{2} \sigma_{v}^{2}+N_{0}}\left[1+\sum_{i=1}^{N_{t}}\left(q_{M_{i}} e^{-j 2 \pi M_{i} k / N}+q_{-M_{i}} e^{j 2 \pi M_{i} k / N}\right)\right] H_{k} e^{j 2 \pi m k / N} \\
= & \frac{1}{N} \sum_{k=0}^{N-1} \frac{\left|H_{k}\right|^{2} \sigma_{v}^{2}}{\left|H_{k}\right|^{2} \sigma_{v}^{2}+N_{0}} e^{j 2 \pi m k / N} \\
& +\frac{1}{N} \sum_{i=1}^{N_{t}}\left[q_{M_{i}} \sum_{k=0}^{N-1} \frac{\left|H_{k}\right|^{2} \sigma_{v}^{2}}{\left|H_{k}\right|^{2} \sigma_{v}^{2}+N_{0}} e^{j 2 \pi\left(m-M_{i}\right) k / N}+q_{-M_{i}} \sum_{k=0}^{N-1} \frac{\left|H_{k}\right|^{2} \sigma_{v}^{2}}{\mid H_{k}^{2} \sigma_{v}^{2}+N_{0}} e^{j 2 \pi\left(m+M_{i}\right) k / N}\right] \\
= & x_{m}+\sum_{i=1}^{N_{t}}\left(q_{M_{i}} x_{m-M_{i}}+q_{-M_{i}} x_{m+M_{i}}\right)
\end{aligned}
$$

where

$$
x_{m}=\frac{1}{N} \sum_{k=0}^{N-1} \frac{\left|H_{k}\right|^{2} \sigma_{v}^{2}}{\left|H_{k}\right|^{2} \sigma_{v}^{2}+N_{0}} e^{j 2 \pi m k / N} .
$$

Based on Equation (B-21), for $m \in\left\{M_{1}, \cdots M_{i}, \cdots, M_{N_{t}}\right\}$, there are the following expressions 


$$
\left\{\begin{array}{l}
q_{M_{1}}=x_{M_{1}}+\sum_{i=1}^{N_{t}}\left(q_{M_{i}} x_{M_{1}-M_{i}}+q_{-M_{i}} x_{M_{1}+M_{i}}\right) \\
q_{M_{2}}=x_{M_{2}}+\sum_{i=1}^{N_{t}}\left(q_{M_{i}} x_{M_{2}-M_{i}}+q_{-M_{i}} x_{M_{2}+M_{i}}\right) \\
\vdots \\
q_{M_{N_{t}}}=x_{M_{N_{t}}}+\sum_{i=1}^{N_{t}}\left(q_{M_{i}} x_{M_{N_{t}}-M_{i}}+q_{-M_{t}} x_{M_{N_{t}}+M_{i}}\right) \\
q_{-M_{1}}=x_{-M_{1}}+\sum_{i=1}^{N_{t}}\left(q_{M_{i}} x_{-M_{1}-M_{i}}+q_{-M_{i}} x_{-M_{1}+M_{i}}\right) \\
q_{-M_{N_{t}}}=x_{-M_{N_{t}}}+\sum_{i=1}^{N_{t}}\left(q_{M_{i}} x_{-M_{N_{t}}-M_{i}}+q_{-M_{i}} x_{-M_{N_{t}}+M_{i}}\right)
\end{array}\right.
$$

Define the following vectors and matrix:

$$
\begin{aligned}
& \mathbf{q}=\left[q_{M_{1}} \cdots q_{M_{N_{t}}} q_{-M_{1}} \cdots q_{-M_{N_{t}}}\right]^{p} \\
& \mathbf{x}=\left[x_{M_{1}} \cdots x_{M_{N_{t}}} x_{-M_{1}} \cdots x_{-M_{N_{t}}}\right]^{t} \\
& \mathbf{C}=\left[\begin{array}{llllllll}
x_{M_{1}-M_{1}} & x_{M_{1}-M_{2}} \cdots & x_{M_{1}-M_{N_{t}-1}} & x_{M_{1}-M_{N_{t}}} & x_{M_{1}+M_{1}} & x_{M_{1}+M_{2}} \cdots & x_{M_{1}+M_{N_{t}-1}} & x_{M_{1}+M_{N_{t}}} \\
\vdots & & & & & & & \\
x_{M_{N_{t}}-M_{1}} & x_{M_{N_{t}}-M_{2}} \cdots & x_{M_{N_{t}}-M_{N_{t}-1}} & x_{M_{N_{t}}-M_{N_{t}}} & x_{M_{N_{t}}+M_{1}} & x_{M_{N_{t}}+M_{2}} \cdots & x_{M_{N_{t}}+M_{N_{t}-1}} & x_{M_{N_{t}}+M_{N_{t}}} \\
x_{-M_{1}-M_{1}} & x_{-M_{1}-M_{2}} \cdots & x_{-M_{1}-M_{N_{t}-1}} & x_{-M_{1}-M_{N_{t}}} & x_{-M_{1}+M_{1}} & x_{-M_{1}+M_{2}} \cdots & x_{-M_{1}+M_{N_{t}-1}} & x_{-M_{1}+M_{N_{t}}} \\
\vdots & & & & & & & \\
x_{-M_{N_{t}}-M_{1}} & x_{-M_{N_{t}}-M_{2}} \cdots & x_{-M_{N_{t}}-M_{N_{t}-1}} & x_{-M_{N_{t}}-M_{N_{t}}} & x_{-M_{N_{t}}+M_{1}} & x_{-M_{N_{t}}+M_{2}} \cdots & x_{-M_{N_{t}}+M_{N_{t}-1}} & x_{-M_{N_{t}}+M_{N_{t}}}
\end{array}\right]
\end{aligned}
$$

then Equation (B-23) can be expressed as

$$
\mathbf{q}=\mathbf{x}+\mathbf{C q}
$$

or

$$
\mathbf{q}=(\mathbf{I}-\mathbf{C})^{-1} \mathbf{x}
$$

where I is a $2 N_{t} \times 2 N_{t}$ identity matrix. 
To simplify the calculation of $\mathbf{C}$, it can be expressed as

$$
\mathbf{C}=\left[\begin{array}{ll}
\mathbf{F} & \mathbf{E} \\
\mathbf{E}^{*} & \mathbf{F}^{*}
\end{array}\right],
$$

where

$$
\mathbf{E}=\left[\begin{array}{llll}
x_{M_{1}+M_{1}} & x_{M_{1}+M_{2}} \cdots & x_{M_{1}+M_{N_{t}-1}} & x_{M_{1}+M_{N_{t}}} \\
\vdots & & & \\
x_{M_{N_{t}}+M_{1}} & x_{M_{N_{t}}+M_{2}} \cdots & x_{M_{N_{t}}+M_{N_{t}-1}} & x_{M_{N_{t}}+M_{N_{t}}}
\end{array}\right]
$$

and

$$
\mathbf{F}=\left[\begin{array}{lllll}
x_{M_{1}-M_{1}} & x_{M_{1}-M_{2}} \cdots & x_{M_{1}-M_{N_{t}-1}} & x_{M_{1}-M_{N_{t}}} \\
\vdots & & & & \\
x_{M_{N_{t}}-M_{1}} & x_{M_{N_{t}}-M_{2}} \cdots & x_{M_{N_{t}}-M_{N_{t}-1}} & x_{M_{N_{t}}-M_{N_{t}}}
\end{array}\right]
$$

By defining

$$
\begin{aligned}
& \mathbf{q}_{1}=\left[q_{M_{1}} \cdots q_{M_{N_{t}}}\right]^{T}, \\
& \mathbf{q}_{2}=\left[q_{-M_{1}} \cdots q_{-M_{N_{t}}}\right]^{T}, \\
& \mathbf{x}_{1}=\left[x_{M_{1}} \cdots x_{M_{N_{t}}}\right]^{T},
\end{aligned}
$$

and

$$
\mathbf{x}_{2}=\left[x_{-M_{1}} \cdots x_{-M_{N_{t}}}\right]^{T},
$$

then Equation (B-27) can be rewritten as

$$
\left[\begin{array}{cc}
\mathbf{I}-\mathbf{F} & -\mathbf{E} \\
-\mathbf{E}^{*} & \mathbf{I}-\mathbf{F}^{*}
\end{array}\right]\left[\begin{array}{l}
\mathbf{q}_{1} \\
\mathbf{q}_{2}
\end{array}\right]=\left[\begin{array}{l}
\mathbf{x}_{1} \\
\mathbf{x}_{2}
\end{array}\right]
$$

From Equation (B-36), we obtain 


$$
\begin{aligned}
& \mathbf{q}_{1}=\left[(\mathbf{I}-\mathbf{F})-\mathbf{E}\left(\mathbf{I}-\mathbf{F}^{*}-\mathbf{F}^{*}\right)^{-1} \mathbf{E}^{*}\right]^{-1}\left[\mathbf{x}_{1}+\mathbf{E}\left(\mathbf{I}-\mathbf{F}^{*}\right)^{-1} \mathbf{x}_{2}\right] \\
& \mathbf{q}_{2}=\left[\left(\mathbf{I}-\mathbf{F}^{*}\right)-\mathbf{E}^{*}(\mathbf{I}-\mathbf{F})^{-1} \mathbf{E}\right]^{-1}\left[\mathbf{E}^{*}(\mathbf{I}-\mathbf{F})^{-1} \mathbf{x}_{1}+\mathbf{x}_{2}\right]
\end{aligned}
$$

Since it can be proved that $q_{-m}=q_{m}^{*}$ according to (B-19) and (B-20). So, actually, calculating either $\mathbf{q}_{1}$ or $\mathbf{q}_{2}$ is sufficient.

\section{B.2 Mean and Variance}

The mean $\mu_{z}(v)$ and variance $\sigma_{z}^{2}$ are calculated as follows:

$$
\begin{aligned}
\mu_{z}(v) & =E\left[z_{n} \mid v_{n}=v\right] \\
& =E\left[\frac{1}{N} \sum_{k=0}^{N-1} P_{k} R_{k} e^{j 2 \pi k n / N}-\sum_{i=1}^{N_{t}}\left(q_{M_{i}} v_{n-M_{i}}+q_{-M_{i}} v_{n+M_{i}}\right) \mid v_{n}=v\right] .
\end{aligned}
$$

For $M_{i} \neq 0, E\left[v_{n-M_{i}} \mid v_{n}=v\right]=0, V_{k}=\sum_{m=0}^{N-1} v_{m} e^{-j 2 \pi k m / N}$ and $E\left[v_{n+M_{i}} \mid v_{n}=v\right]=0$, so

$$
\begin{aligned}
\mu_{z}(v) & =E\left[\frac{1}{N} \sum_{k=0}^{N-1} P_{k}\left(H_{k} V_{k}+W_{k}\right) e^{j 2 \pi k n / N} \mid v_{n}=v\right] \\
& =\frac{1}{N} \sum_{k=0}^{N-1} P_{k} H_{k} \sum_{m=0}^{N-1} E\left[v_{m} \mid v_{n}=v\right] e^{j 2 \pi k(n-m) / N} \\
& =\frac{1}{N} \sum_{k=0}^{N-1} P_{k} H_{k} \sum_{m=0}^{N-1} v \delta_{n-m} e^{j 2 \pi k(n-m) / N} \\
& =v \frac{1}{N} \sum_{k=0}^{N-1} P_{k} H_{k} \\
& =v q_{0} .
\end{aligned}
$$

The variance is

$$
\sigma_{z}^{2}=E\left[\left|z_{n}\right|^{2} \mid v_{n}=v\right]-\left|\mu_{z}(v)\right|^{2}
$$




$$
=E\left[\left|\frac{1}{N} \sum_{k=0}^{N-1} P_{k} R_{k} e^{j 2 \pi k n / N}-\sum_{i=1}^{N_{t}}\left(q_{M_{i}} v_{n-M_{i}}+q_{-M_{i}} v_{n+M_{i}}\right)^{2}\right| v_{n}=v\right]-\left|\mu_{z}(v)\right|^{2} .
$$

And

$$
\begin{gathered}
E\left[\left|\frac{1}{N} \sum_{k=0}^{N-1} P_{k} R_{k} e^{j 2 \pi k n / N}-\sum_{i=1}^{N_{t}}\left(q_{M_{i}} v_{n-M_{i}}+q_{-M_{i}} v_{n+M_{i}}\right)\right|^{2} \mid v_{n}=v\right] \\
=E\left[\frac{1}{N^{2}} \sum_{k=0}^{N-1} \sum_{l=0}^{N-1} P_{k} R_{k} e^{j 2 \pi k n / N} P_{l}^{*} R_{l}^{*} e^{-j 2 \pi n l / N} \mid v_{n}=v\right] \\
-E\left[\frac{1}{N} \sum_{k=0}^{N-1} P_{k} R_{k} e^{j 2 \pi k n / N} \sum_{j=1}^{N_{t}}\left(q_{M_{j}}^{*} v_{n-M_{j}}^{*}+q_{-M_{j}}^{*} v_{n+M_{j}}^{*}\right) \mid v_{n}=v\right] \\
-E\left[\frac{1}{N} \sum_{l=0}^{N-1} P_{l}^{*} R_{l}^{*} e^{-j 2 \pi n l / N} \sum_{i=1}^{N_{t}}\left(q_{M_{i}} v_{n-M_{i}}+q_{-M_{i}} v_{n+M_{i}}\right) \mid v_{n}=v\right] \\
+E\left[\sum_{i=1}^{N_{t}}\left(q_{M_{i}} v_{n-M_{i}}+q_{-M_{i}} v_{n+M_{i}}\right) \sum_{j=1}^{N_{t}}\left(q_{M_{j}}^{*} v_{n-M_{j}}^{*}+q_{-M_{j}}^{*} v_{n+M_{j}}^{*}\right) \mid v_{n}=v\right]
\end{gathered}
$$

The first term of Equation (B-42) is

$$
\begin{gathered}
E\left[\frac{1}{N^{2}} \sum_{k=0}^{N-1} \sum_{l=0}^{N-1} P_{k} R_{k} e^{j 2 \pi k n / N} P_{l}^{*} R_{l}^{*} e^{-j 2 \pi n l / N} \mid v_{n}=v\right] \\
=E\left[\frac{1}{N^{2}} \sum_{k=0}^{N-1} \sum_{l=0}^{N-1} P_{k} P_{l}^{*}\left(H_{k} V_{k}+W_{k}\right)\left(H_{l}^{*} V_{l}^{*}+W_{l}^{*}\right) e^{j 2 \pi(k-l) n / N} \mid v_{n}=v\right] \\
=\frac{1}{N^{2}} \sum_{k=0}^{N-1} \sum_{l=0}^{N-1} P_{k} P_{l}^{*}\left[H_{k} H_{l}^{*} E\left[V_{k} V_{l}^{*} \mid v_{n}=v\right]+E\left[W_{k} W_{l}^{*} \rrbracket e^{j 2 \pi(k-l) n / N} .\right.\right.
\end{gathered}
$$

Since

$$
E\left[V_{k} V_{l}^{*} \mid v_{n}=v\right]=\sum_{a=0}^{N-1} \sum_{b=0}^{N-1} E\left[v_{a} v_{b}^{*} \mid v_{n}=v\right]^{j 2 \pi(l b-a k) / N}
$$

and 


$$
\begin{aligned}
E\left[v_{a} v_{b}^{*} \mid v_{n}=v\right] & = \begin{cases}E\left[v_{a} \mid v_{n}=v\right] E\left[v_{b}^{*} \mid v_{n}=v\right] & \text { if } a \neq b \\
E\left[\left.v_{n}\right|^{2} \mid v_{n}=v\right] & \text { if } a=b\end{cases} \\
& =E\left[\left|v_{a}\right|^{2} \mid v_{n}=v\right] \delta_{a-b} \\
& =\left[|v|^{2} \delta_{a-n}+\sigma_{v}^{2}\left(1-\delta_{a-n}\right)\right] \delta_{a-b} \\
& =\left[\sigma_{v}^{2}+\left(|v|^{2}-\sigma_{v}^{2}\right) \delta_{a-n}\right] \delta_{a-b},
\end{aligned}
$$

Equation (B-43) becomes

$$
\begin{aligned}
& E\left[\frac{1}{N^{2}} \sum_{k=0}^{N-1} \sum_{l=0}^{N-1} P_{k} R_{k} e^{j 2 \pi k n / N} P_{l}^{*} R_{l}^{*} e^{-j 2 \pi n l / N} \mid v_{n}=v\right] \\
= & \frac{1}{N^{2}} \sum_{k=0}^{N-1} \sum_{l=0}^{N-1} P_{k} P_{l}^{*}\left[\begin{array}{c}
\left.H_{k} H_{l}^{*} \sum_{a=0}^{N-1} \sum_{b=0}^{N-1}\left[\sigma_{v}^{2}+\left(|v|^{2}-\sigma_{v}^{2}\right) \delta_{a-n}\right] \delta_{a-b} e^{j 2 \pi(l b-k a) / N}\right] \\
+E\left[W_{k} W_{l}^{*}\right]
\end{array}\right] e^{j 2 \pi(k-l) n / N} \\
= & \frac{1}{N^{2}} \sum_{k=0}^{N-1} \sum_{l=0}^{N-1} P_{k} P_{l}^{*}\left[H_{k} H_{l}^{*} \sum_{a=0}^{N-1}\left[\sigma_{v}^{2}+\left(|v|^{2}-\sigma_{v}^{2}\right) \delta_{a-n}\right] e^{j 2 \pi(l-k) a / N}+N_{0} N \delta_{k-l}\right] e^{j 2 \pi(k-l) n / N} \\
= & \frac{1}{N^{2}} \sum_{k=0}^{N-1} \sum_{l=0}^{N-1} P_{k} P_{l}^{*}\left[H_{k} H_{l}^{*} \sigma_{v}^{2} N \delta_{k-l}+H_{k} H_{l}^{*}\left(|v|^{2}-\sigma_{v}^{2}\right) e^{j 2 \pi(l-k) n / N}+N_{0} N \delta_{k-l}\right] e^{j 2 \pi(k-l) n / N} \\
= & \frac{1}{N} \sum_{k=0}^{N-1} P_{k} P_{k}^{*}\left(\left|H_{k}\right|^{2} \sigma_{v}^{2}+N_{0}\right)+\left(|v|^{2}-\sigma_{v}^{2}\right) \frac{1}{N^{2}} \sum_{k=0}^{N-1} \sum_{l=0}^{N-1} P_{k} H_{k} P_{l}^{*} H_{l}^{*} \\
= & \frac{1}{N} \sum_{k=0}^{N-1}\left|P_{k}\right|^{2}\left(\left|H_{k}\right|^{2} \sigma_{v}^{2}+N_{0}\right)+\left(|v|^{2}-\sigma_{v}^{2}\right)\left|q_{0}\right|^{2} .
\end{aligned}
$$

The second term of Equation (B-42) is

$$
\begin{gathered}
E\left[\frac{1}{N} \sum_{k=0}^{N-1} P_{k} R_{k} e^{j 2 \pi k n / N} \sum_{j=1}^{N_{t}}\left(q_{M_{j}}^{*} v_{n-M_{j}}^{*}+q_{-M_{j}}^{*} v_{n+M_{j}}^{*}\right) \mid v_{n}=v\right] \\
=\frac{1}{N} \sum_{k=0}^{N-1} P_{k} \sum_{j=1}^{N_{t}}\left(q_{M_{j}}^{*} E\left[v_{n-M_{j}}^{*} R_{k} \mid v_{n}=v\right]+q_{-M_{j}}^{*} E\left[v_{n+M_{j}}^{*} R_{k} \mid v_{n}=v\right] e^{j 2 \pi k n / N} .\right.
\end{gathered}
$$

Since 


$$
\begin{aligned}
E\left[R_{k} v_{n-M_{j}}^{*} \mid v_{n}=v\right] & \left.=H_{k} E\left|V_{k} v_{n-M_{j}}^{*}\right| v_{n}=v\right] \\
& =H_{k} \sum_{m=0}^{N-1} E\left[v_{m} v_{n-M_{j}}^{*} \mid v_{n}=v\right]^{-j 2 \pi k m / N} \\
& =H_{k} \sum_{m=0}^{N-1} \sigma_{v}^{2} \delta_{n-M_{j}-m} e^{-j 2 \pi k m / N} \\
& =H_{k} \sigma_{v}^{2} e^{j 2 \pi\left(M_{j}-n\right) k / N}
\end{aligned}
$$

then Equation (B-47) is

$$
\begin{array}{r}
E\left[\frac{1}{N} \sum_{k=0}^{N-1} P_{k} R_{k} e^{j 2 \pi k n / N} \sum_{j=1}^{N_{t}}\left(q_{M_{j}}^{*} v_{n-M_{j}}^{*}+q_{-M_{j}}^{*} v_{n+M_{j}}^{*}\right) \mid v_{n}=v\right] \\
=\sigma_{v}^{2} \frac{1}{N} \sum_{k=0}^{N-1} P_{k} H_{k} \sum_{j=1}^{N_{t}}\left(q_{M_{j}}^{*} e^{j 2 \pi M_{j} k / N}+q_{-M_{j}}^{*} e^{-j 2 \pi M_{j} k / N}\right) \\
=\sigma_{v}^{2} \frac{1}{N} \sum_{k=0}^{N-1} P_{k} H_{k} \sum_{j=1}^{N_{t}}\left(q_{-M_{j}} e^{j 2 \pi M_{j} k / N}+q_{M_{j}} e^{-j 2 \pi M_{j} k / N}\right) .
\end{array}
$$

Similarly, the third term of Equation (B-42) is

$$
\begin{array}{r}
E\left[\frac{1}{N} \sum_{l=0}^{N-1} P_{l}^{*} R_{l}^{*} e^{-j 2 \pi n l / N} \sum_{i=1}^{N_{t}}\left(q_{M_{i}} v_{n-M_{i}}+q_{-M_{i}} v_{n+M_{i}}\right) \mid v_{n}=v\right] \\
=\sigma_{v}^{2} \frac{1}{N} \sum_{k=0}^{N-1} P_{k}^{*} H_{k}^{*} \sum_{j=1}^{N_{t}}\left(q_{-M_{j}} e^{j 2 \pi M_{j} k / N}+q_{M_{j}} e^{-j 2 \pi M_{j} k / N}\right) .
\end{array}
$$

The fourth term of Equation (B-42) is

$$
\begin{array}{r}
E\left[\sum_{i=1}^{N_{t}}\left(q_{M_{i}} v_{n-M_{i}}+q_{-M_{i}} v_{n+M_{i}}\right) \sum_{j=1}^{N_{t}}\left(q_{M_{j}}^{*} v_{n-M_{j}}^{*}+q_{-M_{j}}^{*} v_{n+M_{j}}^{*}\right) \mid v_{n}=v\right] \\
=\sum_{i=1}^{N_{t}} \sum_{j=1}^{N_{t}} E\left[q_{M_{i}} q_{M_{j}}^{*} v_{n-M_{i}} v_{n-M_{j}}^{*}+q_{-M_{i}} q_{-M_{j}}^{*} v_{n+M_{i}} v_{n+M_{j}}^{*} \mid v_{n}=v\right] \\
=\sum_{i=1}^{N_{t}}\left(\left|q_{M_{i}}\right|^{2} E\left[\left|v_{n-M_{i}}\right|^{2} \mid v_{n}=v\right]+\left|q_{-M_{i}}\right|^{2} E\left[\left[\left.v_{n+M_{i}}\right|^{2} \mid v_{n}=v\right]\right)\right.
\end{array}
$$




$$
=\sigma_{v}^{2} \sum_{i=1}^{N_{i}}\left(\left|q_{M_{i}}\right|^{2}+\left|q_{-M_{i}}\right|^{2}\right)
$$

Finally, based on Equation (B-46), (B-49), (B-50) and (B-51), the variance is written as

$$
\begin{aligned}
\sigma_{z}^{2}=\frac{1}{N} \sum_{k=0}^{N-1} \mid & \left.P_{k}\right|^{2}\left(\left|H_{k}\right|^{2} \sigma_{v}^{2}+N_{0}\right)+\left(|v|^{2}-\sigma_{v}^{2}\right)\left|q_{0}\right|^{2} \\
& -\sigma_{v}^{2} \frac{1}{N} \sum_{k=0}^{N-1} P_{k} H_{k} \sum_{j=1}^{N_{t}}\left(q_{-M_{j}} e^{j 2 \pi M_{j} k / N}+q_{M_{j}} e^{-j 2 \pi M_{j} k / N}\right) \\
& -\sigma_{v}^{2} \frac{1}{N} \sum_{k=0}^{N-1} P_{k}^{*} H_{k}^{*} \sum_{j=1}^{N_{t}}\left(q_{-M_{j}} e^{j 2 \pi M_{k} k / N}+q_{M_{j}} e^{-j 2 \pi M_{j} k / N}\right) \\
& +\sigma_{v}^{2} \sum_{i=1}^{N_{t}}\left(\left|q_{M_{i}}\right|^{2}+\left|q_{-M_{i}}\right|^{2}\right)-|v|^{2}\left|q_{0}\right|^{2} \\
=\frac{1}{N} \sum_{k=0}^{N-1} P_{k}^{*} & {\left[P_{k}\left(\left|H_{k}\right|^{2} \sigma_{v}^{2}+N_{0}\right)-H_{k}^{*} \sigma_{v}^{2} \sum_{i=1}^{N_{i}}\left(q_{M_{i}} e^{-j 2 \pi k M_{i} / N}+q_{-M_{i}} e^{j 2 \pi k M_{i} / N}\right)\right] } \\
& -\sigma_{v}^{2} \sum_{j=1}^{N_{t}}\left(q_{-M_{j}} \frac{1}{N} \sum_{k=0}^{N-1} P_{k} H_{k} e^{j 2 \pi U_{j} k / N}+q_{M_{j}} \frac{1}{N} \sum_{k=0}^{N-1} P_{k} H_{k} e^{-j 2 \pi M \mu_{j} k / N}\right) \\
& +\sigma_{v}^{2} \sum_{i=1}^{N_{t}}\left(\left|q_{M_{i}}\right|^{2}+\left|q_{-M_{i}}\right|^{2}\right)-\sigma_{v}^{2}\left|q_{0}\right|^{2}
\end{aligned}
$$

Since $P_{k}\left(\left|H_{k}\right|^{2} \sigma_{v}^{2}+N_{0}\right)-H_{k}^{*} \sigma_{v}^{2} \sum_{i=1}^{N_{t}}\left(q_{M_{i}} e^{-j 2 \pi k M_{i} / N}+q_{-M_{i}} e^{j 2 \pi k M_{i} / N}\right)=H_{k}^{*} \sigma_{v}^{2}$ according to Equation (B-13), $q_{M_{j}}=\frac{1}{N} \sum_{k=0}^{N-1} P_{k} H_{k} e^{j 2 \pi M_{k} / k / N}, q_{-M_{j}}=\frac{1}{N} \sum_{k=0}^{N-1} P_{k} H_{k} e^{-j 2 \pi h_{j} k / N}$ and $q_{-m}=q_{m}^{*}$ according to Equation (B-19) and (B-20), giving

$$
\begin{gathered}
\sigma_{z}^{2}=\frac{1}{N} \sum_{k=0}^{N-1} P_{k}^{*} H_{k}^{*} \sigma_{v}^{2}-\sigma_{v}^{2} \sum_{i=1}^{N_{t}}\left(q_{M_{i}} q_{M_{i}}^{*}+q_{-M_{i}} q_{-M_{i}}^{*}\right) \\
+\sigma_{v}^{2} \sum_{i=1}^{N_{t}}\left(\left|q_{M_{i}}\right|^{2}+\left|q_{-M_{i}}\right|^{2}\right)-\sigma_{v}^{2}\left|q_{0}\right|^{2}
\end{gathered}
$$




$$
\begin{aligned}
& =\sigma_{v}^{2} q_{0}^{*}-\sigma_{v}^{2}\left|q_{0}\right|^{2} \\
& =\sigma_{v}^{2} q_{0}^{*}\left(1-q_{0}\right) .
\end{aligned}
$$

